\title{
Nanoscopy inside living brain slices
}

\author{
Dissertation \\ for the award of the degree \\ "Doctor rerum naturalium" \\ submitted to the Physics Department \\ of the Georg-August-Universität Göttingen \\ by \\ Nicolai Thomas Urban \\ of \\ Englewood, New Jersey, United States of America
}

Göttingen, September 21 $1^{\text {st }}, 2012$ 
Thesis Committee Members:

Prof. Dr. Stefan W. Hell (first reviewer)

Department of Nanobiophotonics, Max-Planck-Institute for Biophysical Chemistry

Prof. Dr. Jörg Enderlein (second reviewer)

Biophysics/Complex Systems Group, III. Insitute of Physics, Georg August University Göttingen

Prof. Dr. Erwin Neher

Department of Membrane Biophysics, Max-Planck-Institute for Biophysical Chemistry

Additional members of the examination board:

Prof. Dr. Tobias Moser

InnerEarLab, Department of Otolaryngology, Göttingen University Medical School

Prof. Dr. Silvio Rizzoli

STED Microscopy of Synaptic Function Group, European Neuroscience Institute

Prof. Dr. Christoph Schmidt

Department of Molecular and Cellular Biophysics, III. Insitute of Physics, Georg August University Göttingen

Date of the oral examination: November $1^{\text {st }}, 2012$ 


\section{Dedication}

To all the people who believed in me, when I could not. 
I hereby verify that I, Nicolai Thomas Urban, was the sole author of the presented dissertation titled "Nanoscopy inside living brain slices" and that I used no other sources or aides than those referenced in the text.

Göttingen, $21^{\text {st }}$ September, 2012 


\section{Abbreviations}

ACSF Artificial Cerebrospinal Fluid: an aqueous solution containing (among others) salts, sugar and $\mathrm{pH}$-buffer, mimicking a natural environment of brain cells. Modified versions can (purposely) vary significantly from physiological solutions, by containing drugs and/or elevated or depleted levels of ions or molecules.

CA1 Cornu Ammonis, part 1: a region of the hippocampus containing a specific type of pyramidal neurons. The name is derived from Cornu Ammonis (horns of Amun, or ram's horns)

CA3 Cornu Ammonis, part 3: a region of the hippocampus containing a specific type of pyramidal neurons.

chem-LTP Chemical Long-Term Potentiation: a chemically induced long-term potentiation effect. See also: $L T P$

CV Correction Value of the objective lens' correction collar. Ranges from one to ten.

EYFP Enhanced Yellow Fluorescent Protein: a green-yellow-emitting fluorescent protein derived from the jellyfish aequorea victoria.

FWHM Full-Width at Half-Maximum: a measure of the width of distribution (such as pulses, Gaussian distributions).

F-Actin Filamentous Actin: the polymerized form of actin.

G-Actin Globular Actin: the monomeric form of actin.

$\boldsymbol{\lambda}$ The wavelength of light, generally measured in $\mathrm{nm}$.

LatB Latrunculin B: a biological toxin derived from red sea sponges latrunculia magnifica. This toxin passively promotes actin filament depolymerization.

LTP Long-Term Potentiation: describes a long-lived enhancement of synaptic strength following specific stimuli. See also: chem-LTP

NA Numerical Aperture: a dimensionless number describing the maximum angle in which an optical element (such as an objective lens) can emit and collect light.

n Refractive Index: details the speed of light inside a medium.

PSF Point Spread Function (of a microscope): describes the system response to imaging a point source, i.e. if a microscope images a point object, this is what the resulting image will look like. The final image will be a convolution of the object and the point spread functions. 
RESOLFT Reversible Saturable Optical Fluorescence Transitions: (a) A general term for targeted optical nanoscopic methods that achieve superresolution by selectively switching between two saturable of switchable molecular states; this nanoscopy family encompasses STED, GSD, SSIM and others. (b) A specific term for RESOLFT microscopy using reversibly switchable fluorescent proteins (RSFPs). This is the definition used in this thesis.

RSFP Reversibly Switchable Fluorescent Proteins: can be switched repeatedly between an excitable (bright) on- and a non-excitable (dark) off-state using optical wavelengths.

STED Stimulated Emission Depletion: a far-field microscopy technique that enables subdiffraction resolution by selectively switching off excited fluorophores in the periphery of the focal spot. The first optical technique developed to provide true subdiffraction resolution, i.e. a purely physical process not relying on computational post-processing.

Thy 1 Thy-1 gene or Thy-1 protein: both are abundant in projection neurons in the hippocampus. 


\section{Table of Contents}

$\begin{array}{lr}\text { 1. Introduction } & \mathbf{1}\end{array}$

1.1. A brief history of optical methods in neurosciences . . . . . . . . 1

1.1.1. Early development of microscopy and labeling techniques . . 2

1.1.2. Fluorescence microscopy . . . . . . . . . . . . . . . . 3

1.2. Optimization of the light microscope . . . . . . . . . . . . 4

1.2.1. Towards shorter wavelengths ............ . 5

1.2.2. Optimizing the focal spot . . . . . . . . . . . 6

1.2.3. Near-field nanoscopic methods . . . . . . . . . . . . 8

1.3. Far-field optical nanoscopy . . . . . . . . . . . . . . . . . . . 9

1.3.1. Targeted nanoscopy techniques (generalized) . . . . . . . 12

1.3.2. Stochastic (pointillistic) nanoscopy techniques . . . . . . 12

1.4. Nanoscopic details in the brain . . . . . . . . . . . . . . . . 14

1.5. Specific aims for this dissertation . . . . . . . . . . . 17

2. Materials and methods $\quad 19$

2.1. Organotypic hippocampal brain slices . . . . . . . . . . . . . 20

2.1.1. Brain slice preparation . . . . . . . . . . . . . 21

2.2. Optical microscopy setups . . . . . . . . . . . . . . . 23

2.2.1. A STED setup for imaging living brain slices . . . . . . . . . . 24

2.3. A RESOLFT microscope for imaging neurons with low light levels . . 29

2.3.1. RESOLFT switching scheme . . . . . . . . . . . 32

2.4. Labeling method, fluorescent proteins and viral transduction . . . . 33

2.4.1. Lifeact: an actin-binding peptide . . . . . . . . . 33

2.4.2. Fluorescent markers for STED and RESOLFT . . . . . . . . 34

2.4.2.1. Reversibly switchable fluorescent proteins (RSFPs) 35

2.4.3. Viral transduction . . . . . . . . . . . . . . . 37 
2.4.4. Buffers and drugs . . . . . . . . . . . . . . . . 39

3. Imaging deep inside neuronal tissue by compensating spherical aberrations 41

3.1. Refractive index of brain tissue . . . . . . . . . . . . . . . . . 42

3.1.1. Estimating the average refractive index of the hippocampus . 43

3.2. Measuring and compensating spherical aberrations . . . . . . . . 45

3.2.1. The correction collar . . . . . . . . . . . . . . . . . 46

3.2.2. Distortions of the microscope point spread functions due to spherical aberrations . . . . . . . . . . . . . . 46

3.2.3. Aberration compensation for confocal and STED imaging . . 49

3.2.4. Optimizing experimental parameters . . . . . . . . . . . 53

3.2.4.1. Coverslip thickness and immersion medium . . . . 53

3.2.4.2. Coverslip tilt and unstable immersion media . . . . 55

3.3. Measuring the spatial resolution deep within brain slices . . . . . . 56

3.3.1. Measuring the spine neck diameter . . . . . . . . . . . 57

3.3.2. Resolution as a function of imaging depth beneath the surface 59

3.4. Discussion . . . . . . . . . . . . . . . . . . . . . 61

3.4.1. Analysis and conclusions . . . . . . . . . . . . 61

3.4.2. Outlook and improvements . . . . . . . . . . . . . . 64

4. Physiological nanoscopy of dendritic spines and actin substructures 67

4.1. Lifeact label . . . . . . . . . . . . . . . . . . . . . . . . 68

4.1.1. Lifeact and cytosolic labeling compared . . . . . . . . . . 69

4.2. Imaging paradigms . . . . . . . . . . . . . . . . . . 71

4.3. Latrunculin B effects on Lifeact-labeled neurons . . . . . . . . . . . . 72

4.4. Actin structures in dendritic spines . . . . . . . . . . . . . . 75

4.4.1. Spine heads . . . . . . . . . . . . . . . . . . 75

4.4.2. Spine necks . . . . . . . . . . . . . . . . . 78

4.4.3. Dendritic shafts . . . . . . . . . . . . . . . . . 82

4.5. Spine motility and spontaneous actin dynamics . . . . . . . . 83

4.5.1. Rapid morphological changes . . . . . . . . . . . . . . 85

4.5.2. Fast motility and movement . . . . . . . . . . . . 86

4.5.3. Effects of heating . . . . . . . . . . . . . . . 89 
4.5.4. Continuous time-lapse imaging and cell viability . . . . . . 89

4.6. Long-term potentiation . . . . . . . . . . . . . . . . . . . . . . 91

4.7. Analysis and discussion . . . . . . . . . . . . . . . . . 95

4.7.1. Morphological background label . . . . . . . . . . . . . 95

4.7.2. Bleaching ...................... 96

4.7.3. Depth penetration for RESOLFT imaging . . . . . . . . 97

4.7.4. Low light exposure with RESOLFT . . . . . . . . . . . . 97

4.7.5. Imaging speed . . . . . . . . . . . . . . . 98

4.7.6. Viral transfection . . . . . . . . . . . . . . . . . . . 99

4.7.7. Influence of optical recordings on neuronal health or dynamics? . . . . . . . . . . . . . . . . . . . . 99

4.7.8. Application of potentiation medium or Latrunculin solution . 100

4.7.9. Plasma clot acting as diffusion barrier? . . . . . . . . . . . 101

4.7.10. Long-term potentiation . . . . . . . . . . . . . . 101

4.7.11. Inhibition of motility and morphological changes by Latrunculin ...................... 103

4.7.12. Fast spine motility . . . . . . . . . . . . . . . . 104

4.7.13. Influence of heating on spine motility . . . . . . . . . 104

4.7.14. Differential motility of spine heads and spine necks . . . . 105

4.7.15. Functional significance of morphological changes of spine necks . . . . . . . . . . . . . . . . 106

4.7.16. Is STED or RESOLFT better suited for neuronal imaging? . . 107

$\begin{array}{lr}\text { 5. Conclusions and outlook } & 109\end{array}$

$\begin{array}{ll}\text { Bibliography } & 113\end{array}$

$\begin{array}{lr}\text { A. Appendix } & 123\end{array}$

$\begin{array}{ll}\text { Acknowledgments } & 126\end{array}$ 



\section{Introduction}

\subsection{A brief history of optical methods in neurosciences}

When facing an unknown object our first reaction usually is to step back and take a closer look. Surely, visual inspection is one of the oldest tools used by man to understand the nature of his surroundings, and there is a certain charm in the simplicity and intuitiveness of examining something by sight. Much can be deduced by examining the structure and layout of an object. What is its shape, size and consistency? How does it move and change over time? And in the case of living objects: how does it behave and react? It is therefore not surprising that optical tools have been pivotal to unraveling the mysteries of the brain and the nervous system throughout the history of man. The first documented description and discussion of the nervous system was written in 1700 BC by an ancient Egyptian surgeon, and was based on his experiences and insights gained from dissecting human bodies. ${ }^{1}$ At around $400 \mathrm{BC}$ the renowned Greek philosopher Hippocrates, today considered to be the 'father of medicine', postulated that the brain is the seat of intelligence and that it is involved with sensations. ${ }^{1}$ Slowly, more details of the brain were discovered as individual surgeons and natural philosophers studied the appearance and consistency of the brain. Yet lacking any advanced optical tools they were confined to examining macroscopic structures visible to the naked eye or possibly through a single lens.'

'The oldest discovered lens is 3.000 years old and originates from ancient Assyria. Lenses and their function were known in ancient Egypt, Greece and Rome. 


\subsubsection{Early development of microscopy and labeling techniques}

This all changed dramatically with the turn of the $16^{\text {th }}$ century and the invention of the compound light microscope. The power to resolve structures on the millimeter and micrometer scale led to the discovery of the cell (in 1665 by Robert Hooke), allowed the formulation of the cell theory ("All living matter consists of cells, which are the basic building blocks of life" as formulated by Theodor Schwann and Matthias Jakob Schleiden in 1839, and "all existing cells are formed from pre-existing cells" as phrased in 1855 by Rudolf Virchow), and enabled the microscopic examination of cellular structures such the nucleus or nerve fibers (in 1717 by Antoni van Leeuwenhoek, who is often called the "first microbiologist"). Yet the improved spatial resolution of the available optical tools did not change the fact that the brain was a structure with low inherent contrast, making it difficult to discriminate specific details. Without additional methods to provide higher contrast the available imaging techniques were limited in their capacity to visualize interesting structures. Further complications stemming from the difficulty of sustaining organic tissue needed to be overcome by developing fixation methods (using alcohol or later formaldehyde) for preserving organic structures. These fixation methods were complemented by techniques for cutting and preparing thin slices of fixed organic tissue. Many novel cell staining techniques were developed in the $19^{\text {th }}$ and $20^{\text {th }}$ century, which greatly aided the progression of the neurosciences. The Nissl staining that was developed at the end of the $19^{\text {th }}$ century helped visualize cell bodies (and the endoplasmatic reticulum), which enabled the study of the cytoarchitecture of the brain: the known segments of the brain could again be divided into subdivisions. The myelin-sheath stainings and the subsequent silver impregnation techniques that were developed in the 1950s allowed scientists to follow axons and fiber tracts through the brain and thereby to unravel the organization of the nervous system. Another milestone was the development of Camillo Golgi's staining method (la reazione nera). Golgi's method only stained a small, apparently random subset of cells, but these were stained completely: axons, dendrites and all. Such isolated cells offered very high contrast that could be examined in great detail. This staining method was adopted and later perfected by Santiago Ramòn y Cajal, allowing him to conduct paradigm-shifting research of the central nervous system. He 
was the first to describe dendritic spines, which are small protrusions located on neuronal dendrites and which were dismissed by his contemporary Camillo Golgi as being staining artifacts. Cajal also produced detailed functional circuit diagrams of the hippocampus, which led him to propose his theory of dynamic polarization ("Neuronal signals are received by dendrites and sent by axons").

\subsubsection{Fluorescence microscopy}

The emergence of fluorescent labeling and microscopy methods in the early 1900s laid the path for the study of living brain cells and their dynamics. By introducing fluorescent dyes into cells and tracking the movement of the dye, axonal transport phenomena were observed, enabling the axonal pathways to be mapped much more accurately." Fluorescent microscopy really kicked off with the invention of antibody labeling techniques in 1941 and the subsequent recognition in 1974 that these antibodies could not only be used for visualizing immune responses, but could target and label all sorts of proteins such as the ubiquitous actin and tubulin. ${ }^{2}$ Proteins could be labeled by creating a primary antibody that targeted a specific protein of interest and then adding a secondary antibody fused to a fluorescent dye, which then attached itself to the primary antibody, thereby illuminating the structure of interest. This immunostaining technique, which is still widely used today, facilitated the observation of virtually any protein of interest. By using multiple sets of antibodies, each with a differently colored fluorescent dye, colocalization experiments were possible, in which the locations of multiple sets of proteins could be determined simultaneously and in relation to each other. The observation of electrical activity in live neurons with fluorescent microscopy was possible with the development of calcium-sensitive ${ }^{3}$ and voltage-sensitive dyes ${ }^{4}$ around 1980 . By the end of the $20^{\text {th }}$ century, widefield fluorescence microscopy had become one of the dominating techniques in fundamental neuroscience. ${ }^{5}$

\footnotetext{
"Concomitant with fluorescent methods, radioactive staining and tracing methods that were viable for live-cell recordings, at least over short times, were developed and implemented
} 


\subsection{Optimization of the light microscope}

The development and optimization of the compact light microscope progressed continuously from its first implementation in 1590. Chromatic and spherical aberrations were the main limitations that needed to be overcome by crafting lenses of higher quality and arranging them in a better fashion. This process was tenuous and involved much trial and error. All this changed dramatically when the microscope producer Carl Zeiss, the chemist and glass technologist Otto Schott and the physicist Ernst Abbe teamed up to develop the optimal light microscope. Zeiss and Abbe realized that a solid theoretical framework was necessary to improve and streamline the development of microscope optics. After considerable research Ernst Abbe derived his wave theory of microscopic imaging ${ }^{6}$ and in doing so made a farreaching discovery: there is a fundamental limit to the minimal size a spot of light can be focused, which depends only on the microscope optics and the wavelength of light $\lambda$ that was used. Given the Rayleigh criterion for spatial resolution, Abbe found that two objects cannot be resolved if they are within a distance $d$ or less of each other:

$$
d=\frac{\lambda}{2 n \sin (\alpha)}=\frac{\lambda}{2 \mathrm{NA}}
$$

Here $n$ is the refractive index of the objective immersion medium and $\alpha$ the semiaperture angle of the objective lens. Both values are defined by the design of a given objective lens and are commonly abbreviated as the numerical aperture $\mathrm{NA}:=n \sin \alpha$. The numerical aperture is limited by definition, ${ }^{\prime \prime \prime}$ and in practice does not exceed NA $=0.95$ for dry (air) objective lenses and NA $=1.45$ for very high quality oil immersion objective lenses. The refractive index of typical immersion media lies between $1 \leq n \leq 1.7$, with $\mathrm{n}=1$ for air, $\mathrm{n}=1.33$ for water, $\mathrm{n}=1.46$ for glycerol and $\mathrm{n}=1.51$ for specialized immersion oil (which matches the refractive index of glass). Given the restrictions imposed by the optical components this limits the size and distance in which structures can be resolved with visible light (390 $\mathrm{nm} \leq \lambda \leq 750 \mathrm{~nm})$ to $\Delta d_{x, y} \approx 190 \mathrm{~nm}$ in the optical (x,y) plane and

\footnotetext{
IIIBecause $\sin \alpha \leq 1$, it follows that $\mathrm{NA} \leq \mathrm{n}$.
} 
$\Delta d_{z} \approx 390 \mathrm{~nm}$ along the optical $(\mathrm{z})$ axis. ${ }^{\mathrm{IV}}$ All structures that are closer than this distance $\Delta d$ would appear blurred together when imaged.

\subsubsection{Towards shorter wavelengths}

Abbe's diffraction limit went mostly uncontested for a long time, until scientists began pushing at the edges and trying to find ways to circumvent the law. The obvious points of attack here are using shorter wavelengths and somehow improving the numerical aperture. The use of shorter wavelengths lying outside the visible spectrum is not without difficulty, however. Ultraviolet (UV) light is lethal to living cells, a fact that severely limits the use of UV light for live cell observation. There are also technical difficulties, since only very few materials are transparent for UV light. Most materials do not have a large enough energy gap to avoid absorption of high-energy UV photons. Furthermore, Rayleigh scattering is more severe for UV light, as it increases with $\lambda^{-4}$. These are some of the reasons why it is difficult to fabricate UV lenses and as a result to focus UV light.

A major breakthrough in achieving high spatial resolution by reducing the wavelength came with the invention of the electron microscope (EM) in 1931 by Max Knoll and Ernst Ruska. ${ }^{7}$ An electron microscope exploits the wave character of electrons, whose de Broglie wavelength is more than five orders of magnitude shorter than that of visible photons. Consequently, electron microscopy enables spatial resolutions on the atomic scale. With this sudden leap in resolving power all kinds of previously unobservable neuronal structures could be properly visualized for the first time. One result obtained with electron microscopy was the detailed observation of the synaptic cleft that divides the pre- and postsynaptic parts of a synapse. ${ }^{8}$ Because pre- and postsynapse could clearly be discriminated as separate entities, Cajal's neuron doctrine was verified, which had postulated that brain cells are individual entities and not one continuous network. ${ }^{\vee}$ Further discoveries obtained with electron microscopy entailed the discrimination of excitatory and inhibitory synapses ${ }^{9}$ and the observation of nanoscopic subcellular components such as synaptic vesicles and the spine apparatus. ${ }^{10}$ Electron microscopy is not

\footnotetext{
${ }^{\mathrm{IV}}$ calculated using $\lambda=490 \mathrm{~nm}$, as used in this thesis

${ }^{\vee}$ This had been a hotly contested question between Golgi and Cajal, which was even disputed in public during Camillo Golgi's Nobel Lecture in 1906.
} 
without drawbacks, however. Not only is the focused electron beam highly destructive to biological tissue, but samples are generally imaged under vacuum conditions and must undergo various fixation procedures to preserve the tissue and increase the contrast. These factors are highly prohibitive for living samples. Another disadvantage of electron microscopy is its restricted ability to examine only surfaces and thin samples. This limitation can be overcome, however, in a fashion that allows entire blocks of fixated neuronal tissue to be observed with electron microscopy. First, the surface layer of a fixated tissue sample is examined with EM before shaving off this layer with a precision microtome. Subsequently, the new surface layer can be imaged and the procedure repeated until the entire brain preparation has been imaged, layer by layer. This procedure has successfully been implemented to reconstruct large-scale neural networks ${ }^{11}$ in a block of fixed brain tissue by painstakingly tracing individual fibers from image to image.

\subsubsection{Optimizing the focal spot}

A different approach for pushing the diffraction limit a little further came with the invention of confocal microscopy by Marvin Minsky in 1957. Confocal microscopy utilizes a point-like detection, ${ }^{\mathrm{VI}}$ which has two main effects. It blocks fluorescence signals from out-of-focus sources, thereby limiting the recorded fluorescence to whatever was emitted in the central focal region. This allows optical sectioning, which means that image planes can be recorded sequentially at intervals along the optical axis, allowing the reconstruction of three-dimensional images. Furthermore, the point-spread function (PSF) of the microscope is now not just the PSF of the beam alone, but a convolution with the detection PSF, which results in a $\sqrt{2}$ improvement of the resolution over widefield fluorescence microscopy. Confocal microscopy in its original implementation was not suitable for biological research as it was far too slow and unstable. The development of lasers, desktop computers and fast, stable scanning techniques allowed it to be enhanced sufficiently to be interesting for biological experiments, resulting in the development of the confocal scanning laser microscope. ${ }^{12,13}$

\footnotetext{
${ }^{\mathrm{VI}}$ The terminology "confocal pinhole" is frequently used.
} 
The two-photon microscope invented by Winfried Denk et al. in $1990^{14}$ heralded another paradigm shift for the use of optical microscopy in the neurosciences in regards to tissue penetration depth. Two-photon processes had been theoretically predicted in the $1930 \mathrm{~s},{ }^{15}$ but could not be experimentally verified until the invention of the laser. ${ }^{16}$ Standard fluorescence microscopy involved single-photon processes, in which a single photon is energetic enough to lift a fluorophore into an excited state upon absorption. In contrast, two-photon microscopes use wavelengths that are severely red-shifted, so that single photons do not have enough energy to excite a fluorophore, ${ }^{\mathrm{VII}}$ but two photons do. So if two photons can be absorbed almost simultaneously by the dye (the time window for this is of the order of hundreds of attoseconds), then fluorescence is possible. In principle, multiphoton processes involving more than two photons are possible, but they are exceedingly rare. Twophoton microscopes have several advantages over single-photon microscopes. Twophoton excitation occurs only very close to the focal plane, because a very high photon density is necessary for there to be a reasonable probability of two-photon absorption; the probability scales with the square of the intensity, $\mathrm{I}^{2}$. Therefore, the excitation spot is tightly confined to the focal plane, where the photon density is highest. The fluorescence signal along the optical axis decays with $(\Delta z)^{4}$. This confinement of the fluorescence excitation facilitates axial sectioning, similar to confocal microscopy. But because no confocal detection pinhole is required for this, two-photon microscopes can afford a more 'generous' photon detection. Instead of rejecting all out-of-focus photons as in confocal microscopy, two-photon microscopes can confidently use any photon incident upon the objective lens, because the origin of these photons is known: they must have originated from the immediate vicinity of the focal spot. Two-photon microscopes cause almost no out-of-focus excitation, ${ }^{\mathrm{VIII}}$ so very little bleaching occurs outside the focal spot. This also results in reduced phototoxicity. In the focal spot, however, the very high intensity induces strong bleaching. Two-photon microscopy commonly utilizes deep red or infrared illumination, which reduces scattering and absorption in tissue and allows deeper tissue penetration. ${ }^{17}$ For these reasons two-photon microscopy has become the optical method of choice in neuroscience for examining thick tissue preparations such as brain slices and especially in vivo brains. ${ }^{18}$ The ability to peek

\footnotetext{
VIIThe energy $E$ of a photon is given by $E=\frac{h c}{\lambda}$ with Planck's constant $h$ and the speed of light $c$. ${ }^{\mathrm{VIII}}$ This is no longer true if the excitation laser power is increased beyond certain levels.
} 
into the brains of living animals and watch dynamic processes as they happen is remarkable and potentially allows the combined study of learning, experience and conditioning together with structural changes of the neural network and the structure of individual neurons.

In 1992 it was proven that the focal spot could be constricted even further along the optical axis by using not one, but two opposing objective lenses simultaneously. In this fashion the techniques dubbed $4 \mathrm{Pi}$ microscopy ${ }^{19-22}$ and $\mathrm{I}^{5} \mathrm{M}^{23,24}$ increase the (theoretically possible) solid angle of the microscope from $2 \pi$ to $4 \pi$. They rely on the interference of the opposing beams of light along the optical axis to improve the z-resolution up to sevenfold, achieving axial resolutions between $100 \mathrm{~nm}$ to $150 \mathrm{~nm}$. But even though these increases in spatial resolution were considerable, they were not (in theory) unlimited. Crudely put, these techniques only provided a method of creating an improved focal spot. The diffraction limit, therefore, was not broken but merely shifted; there still was a hard lower limit to the spatial resolution that could only be overcome by shorter wavelengths.

\subsubsection{Near-field nanoscopic methods}

For completeness we shall mention alternative methods that have been developed to measure structures on the nanometer or even the atomic scale. These methods, mostly scanning probe techniques, can provide nigh unsurpassed spatial resolution, but are all limited to being surface-sensitive methods only. Scanning tunneling microscopy ${ }^{25}$ uses the extreme distance sensitivity of the tunneling current that can occur between two conducting surfaces separated by a potential barrier (typically a short distance of air or vacuum). Its atomic resolution can be used to determine the topography of conducting surfaces (or samples on conducting surfaces) with great precision, for example. Scanning force microscopy ${ }^{26}$ can be used on conducting and non-conducting surfaces alike, and is therefore also suitable for biological experiments. ${ }^{27}$ Scanning near-field optical microscopy ${ }^{28}$ exploits the properties of evanescent electromagnetic waves that can only be measured in distances shorter than the wavelength of the used light. Solid-immersion objective lenses ${ }^{29}$ offer increased resolution by increasing the refractive index of the objective immersion medium (here a solid), thereby enhancing the numerical aperture of the objective 
lens. This method is not strictly speaking a surface method. The large discrepancy in refractive index between the objective lens immersion material and any biological sample causes severe aberrations, however, limiting the imaging process to the utmost surface layer. So despite their superior resolution, these techniques cannot provide any details on the interior of (living) cells or even function inside tissue.

\subsection{Far-field optical nanoscopy}

Abbe's theoretical concept of the diffraction limit has proved robust despite many attempts to push its boundaries towards smaller values. To date it is not possible to focus a spot of light tighter than predicted by the diffraction limit. So is there no way of breaking this hard limit to spatial resolution? It turns out that there is, but it requires approaching the problem from a completely different angle. As stated above, spatial resolution is limited, because two structures that lie within the diffraction limit $d$ cannot be distinguished if they cannot be excited or detected separately. But assuming these structures were labeled in a different color, for example, they could readily be distinguished chromatically, even if they resided within a distance $d$ of each other. Along the same train of thought, if neighboring structures could be switched on sequentially in time, then they could also be distinguished. What this implies is that not the shrinking of the actual focal spot is the key to limitless resolution but the switching of neighboring structures, so that they are visible at different points in time. This can be done either by forcing all unwanted structures to be switched off or by activating only a certain subset to begin with. But how to switch activated structures off? This was demonstrated in 1994 by Stefan W. Hell and Jan Wichmann with the working concept of stimulated emission depletion (STED) fluorescence microscopy. ${ }^{30}$ In conventional fluorescence microscopy fluorescent dye molecules are excited by irradiation with light of a certain wavelength. The dye molecules are then elevated into an excited state, in which they typically remain for several nanoseconds before randomly dropping down to the ground state by emitting a fluorescence photon. This fluorescence photon is red-shifted (i.e. less energetic) in regards to the initial excitation photon as some energy is converted via nonradiative relaxation processes (vibrations). There is a second way to de-excite a fluorophore, namely by inducing a stimulated emission. This can occur if a second incident photon interacts with the already excited 
fluorophore and induces it to emit a photon. Such a stimulated emission photon is an identical copy of the incident photon. By selecting the wavelength of the de-exciting photons to be outside the spectral range of the detected fluorescence photons, spontaneous $\mathrm{s}^{\mathrm{IX}}$ and stimulated photons can be discriminated chromatically. Typically the de-exciting wavelength is red-shifted in regards to the excitation and the fluorescence emission peaks, so as to not accidentally re-excite the fluorophores with the de-exciting photons. In order to de-excite the excited fluorophores before they emit a fluorescence photon, the de-exciting photon must interact with the fluorophore in the only nanosecond-long lifetime of the excited state. Because the de-excitation (or: off-switching, depletion) is a statistical process, a high number of de-exciting photons are required to ensure that a majority of the excited fluorophores are switched off. The beam intensity required to switch off all but $\frac{1}{e}$ of the excited fluorophores is defined as the saturation intensity $I_{s} .{ }^{\mathrm{X}}$ The probability that a fluorophore remains in the excited state decreases exponentially with the intensity $I$ of the de-excitation beam, so proportional to $\exp \left(-I / I_{s}\right) \cdot{ }^{32}$ If the intensity $I$ of the de-excitation beam exceeds the saturation intensity $I_{s}$, than the chance to de-excite a given fluorophore is $>63 \%$. If we increase the de-excitation intensity to more than $5 I_{s}$, then the probability to switch off the fluorophore is $>99 \%$. Assuming the lifetime of the on-state is very short, then a high photon flux is required to confidently switch off enough fluorophores in the given short time window. ${ }^{\mathrm{X}}$ Conversely, if the on-state is long-lived, much fewer photons per time are required. Also, if the off-state is long-lived, the photo-induced switch will be more durable. It follows that the saturation intensity is inversely proportional to the lifetime of the two on-off states: $I_{s} \propto \frac{1}{\tau_{o n, o f f}}$. The missing proportionality 'constant' in this equation is the optical cross-section of the de-excitation transition $\sigma$; we can define the saturation intensity is $I_{s}:=\frac{1}{\sigma \tau_{o n, o f f}}$. The saturation intensity $I_{s}$ is characteristic for a given dye and varies with the wavelength of the de-excitation beam.

But simply depleting the excited (on-)state is not sufficient for breaking the diffraction limit. A small subset of fluorophores must remain activated, otherwise

\footnotetext{
$\mathrm{IX}_{\text {i.e. fluorescence photons }}$

${ }^{\mathrm{x}}$ The saturation intensity $I_{s}$ is sometimes defined as the intensity required to switch of exactly $\frac{1}{2}$ of the excited fluorophores. ${ }^{31}$

${ }^{\mathrm{XI}}$ Typically several $\mathrm{ns}$ for fluorophores in the first excited state
} 
there will be no fluorescence signal to be recorded. In STED this is done by imprinting a special phase pattern on the wavefront of the de-excitation beam. By passing the de-excitation beam through a (helical) phase plate, the light from different angular locations is phase-shifted in such a manner that creates a perfectly dark area in the center when the beam is focused. This central singularity is possible, because light from opposing sides of the beam cancel each other out due to destructive interference. This generates a for STED typical donut-shaped beam pattern. If such a de-excitation beam is superimposed on top of a standard, Gaussian-shaped excitation beam, then every previously excited fluorophore will be switched off except for those in the immediate center of the donut beam. As there are no de-excitation photons in the center, these fluorophores will be unaffected and can emit fluorescence photons. To create an entire image the two beams are synchronously scanned over the entire region of interest, first exciting and then de-exciting the fluorophores one pixel at a time. The size of the spot in which fluorophores remain activated is defined by the precise shape of the de-excitation donut and decreases with the rising intensity of the de-excitation beam. This STEDgenerated point-spread function can be considered to be an 'effective focal spot' of size $d_{S T E D}$ :

$$
d_{S T E D}(I)=\frac{\lambda}{2 \mathrm{NA} \sqrt{1+a\left(I / I_{s}\right)}}
$$

Here, $a>0$ is the parameter that takes the precise shape of the de-excitation beam into consideration. As can be seen from Equation (1.2), the size of the effective focal spot no longer has a hard limit. Moreover, the effective focal spot can asymptotically be reduced to zero by increasing the intensity $I$ of the de-excitation beam. The diffraction limit, therefore, can be considered not only to have shifted but to have been broken completely. Microscopy techniques that offer diffraction-unlimited resolution are commonly referred to as super-resolution microscopy or nanoscopy techniques. Just as microscopy enabled the examination of structures on the micrometer scale, nanoscopy allows the examination of structures on the nanometer scale, unlimited by diffraction. In practice, there still is a soft limit to the achievable resolution, depending on laser power and the dyes that are available, as well as on the imaged sample. Living neurons, for example, will not be able to tolerate 
arbitrarily high laser powers; also, photodamage, phototoxicity and bleaching will increase with ever rising laser powers.

\subsubsection{Targeted nanoscopy techniques (generalized)}

Nanoscopy techniques that utilize patterned beams, such as STED, have been generalized under the acronym RESOLFT or Reversible Saturable OpticaL Fluorescent Transitions. ${ }^{31,33,34}$ These so-called targeted approaches encompass STEDlike techniques that use different sets of on-off states as their molecular switch, such as depleting the ground state by transferring fluorophores into a longlived, dark triplet state (ground-state depletion or $\mathrm{GSD}^{35}$ ) or by employing photoswitchable fluorescent dyes that can be transferred between chemical states with different fluorescent properties. ${ }^{36}$ This last technique is confusingly also referred to as 'RESOLFT' nanoscopy, in particular when the molecular switching method entails reversibly switchable fluorescent proteins (RSFPs). ${ }^{37,38}$ In this thesis, we will also be referring to this specific method as RESOLFT (nanoscopy). The RSFPs used in the RESOLFT approach can be switched into a very long-lived dark state (large $\tau_{o f f}$ ), so that the saturation intensities required are five to six orders of magnitude less than for STED. This potentially enables nanoscopy using very low light levels. Another targeted nanoscopy method using patterned beams is called saturated pattern excitation $\left(\mathrm{SPEM}^{39}\right)$ or saturated structured illumination $\left(\mathrm{SSIM}^{40}\right)$ microscopy. These methods depopulate the ground state to generate a negative image and instead of donut-shapes they employ patterned grids of ultra-sharp intensity maxima. Despite different names, acronyms and variable sets of onoff states, the fundamental switching principle between saturable or switchable fluorescent states (RESOLFT) is identical for all the aforementioned nanoscopy methods.

\subsubsection{Stochastic (pointillistic) nanoscopy techniques}

There is another school of nanoscopy (based on the same fundamental switching principle) that relies on stochastic on and off-switching for achieving subdiffraction resolution. Stochastic methods depart from the deterministic principle of deliberately confining the fluorescence to a tight, well-known spot, the position 
of which is predetermined by the implemented scanning mechanism. Instead, a widefield illumination scheme is used that only switches on a very few fluorophores at random (for instance by using very dim light). This on-switching or activation is not to be confused with fluorescent excitation. Moreover, activated fluorophores possess the capability to be excited. This activation can be a process similar to RESOLFT with photoswitchable fluorescent proteins, or can be generated by individual fluorophores dropping out of the long-lived triplet states back into the (excitable) ground-state. Once they are activated, fluorophores must be excited strongly ${ }^{\mathrm{XII}}$ and brought to fluoresce as many photons as possible, the signals of which are recorded using a widefield detection scheme (i.e. a camera). If such activated fluorophores are located further apart than the diffraction limit, then they will appear as isolated discs the size of the diffraction limit on camera. As long as the discs do not overlap, the recorded signals can be used to calculate the 'center of mass' of the fluorescent spot, thereby localizing the point of origin with high precision. The precision with which the exact locations can be calculated depends on the number $N$ of collected photons per fluorophore, transforming the 'square-root law' of subdiffraction resolution to $d=\frac{\lambda}{2 \mathrm{NA} \sqrt{N}}$. Once a fluorophore has been recorded it is switched off; this is done either permanently by bleaching or by switching the fluorophore back into its dark state. The entire process is repeated until (at best) all available fluorophores have been excited, recorded and located. These stochastic techniques, known as (F)PALM ${ }^{41,42}$, STORM $^{43}$, GSDIM $^{44}$, dSTORM $^{45}$, PALMIRA ${ }^{46,47}$, etc. have proved impressively that they are equally capable of imaging structures with nanoscale resolution.

Both the stochastic and the targeted methods have distinct advantages, which make them suitable for different tasks and different needs. Stochastic methods have the main benefit of being very simplistic in their optical (widefield) configuration and are therefore less challenging to construct than targeted RESOLFT-like approaches. Widefield detection schemes, however, limit the capability for optical sectioning and background discrimination. Scattering can also severely distort the recorded signal. Stochastic approaches therefore typically either use very thin preparations (thus reducing background sources) for imaging or utilize TIRF-like (total internal reflection fluorescence) illumination schemes, which restrict fluorescent signals

\footnotetext{
$\mathrm{XII}$.e. excited as frequently as possible
} 
to the immediate vicinity of the sample surface. Improved depth resolution can be gained by generating deliberate astigmatism, which distorts the recorded fluorescent discs into ovals of varying shape depending on their precise depth within the sample. ${ }^{48} \mathrm{~A}$ further benefit of stochastic methods is the fact that fluorophores only need to be switched on once, as opposed to RESOLFT techniques in which they must be switched on and off repeatedly. The implemented fluorophores can exhibit high switching fatigue as long as they are very bright and able to emit a high number of photons in a very short time. In contrast, RESOLFT dyes need very low switching fatigue but do not need to be so bright. Because at each imaging location (pixel) entire ensembles of fluorophore are excited and read out simultaneously, not just one but multiple fluorophores can contribute to the recorded signal. Stochastic nanoscopy methods require very specific dyes (or in the case of GSDIM special ambient chemical conditions), thereby restricting the application of these methods in living organisms. Furthermore, it prevents the use of common transgenic animal strains that express standard fluorescent proteins. In this regard STED has an advantage, as it can be used with most of the well-known fluorescent labels and fluorescent proteins available. The speed with which stochastic methods can record images is also highly dependent on the achievable recording speeds of the utilized detection cameras and on the brightness of the fluorescent labels. Because effectively single molecules are being localized, stochastic methods like PALM can follow the trajectory of individual (activated) molecules. ${ }^{49}$ Targeted RESOLFT-like approaches have a distinct advantage as by recording pixels sequentially they can easily detect motion artifacts and are not as susceptible to artifacts introduced by very fast moving structures. Furthermore, the point-detectors that are commonly utilized (but are not required) in targeted approaches are beneficial for background suppression and optical sectioning.

\subsection{Nanoscopic details in the brain}

Can nanoscopy play an important role in brain sciences comparable to microscopy, as was outlined in our brief history of optical methods in the neurosciences? One central question of neuroscience today is just how memories and experiences can be stored in the brain. In what form are they preserved - chemical deposits, electrical patterns, synaptic networks or morphological changes? This question becomes even 
more complex when considering that there is not just one but several different types of memories. We can distinguish between short- and long-term memory, declarative memory (recollection of events and facts), procedural memory (commonly learned tasks, muscle-memory, primed responses) autobiographic memory and many other subcategories. How are they processed, where and when are they stored, when are they forgotten? In current opinion, short-term memories are saved in the form of chemical and electrical signals passed from synapse to synapse. At some point they are either forgotten or else processed and stored elsewhere in the brain, probably in the form of synaptic plasticity. That means in the creation of new synaptic junctions between neurons or in the modification of the strength of existing synapses. How can the strength of an individual synaptic connection be altered? For this, we need to know what a typical synapse looks like. Most excitatory synapses ${ }^{\mathrm{XIII}}$ are located on dendritic spines, which are small protrusions on the neuronal dendrites. Most dendritic spines consist of a bulbous head connected to the parent dendrite through a slender spine neck, but the proportions of spine head and neck vary considerably from spine to spine. A lasting synapse can be formed when a dendritic spine contacts a varicosity (or: bouton) on a nearby axon. At such a synapse, an electrical signal arriving from the axon can be relayed via a chemical signal to the dendritic spine, which can itself create an electrical signal in the adjoining dendrite. The axonal bouton is said to form the presynaptic part of the connection and dendritic spines the postsynaptic part. So if synapses are junctions that transmit electric and chemical signals, then how can their precise functionality be modified? There are many different pathways by which this might be accomplished, for example by increasing the number of proteins or synaptic vesicles at the synaptic active zone, increasing the surface contact area of the synapse or modifying the overall size and geometry of the dendritic spine.

While there is general agreement that a number of different factors contribute to changes in synaptic plasticity, it has become quite clear that morphological changes of nanoscopic structures especially in dendritic spines play an important role, too. However, these changes are very difficult to observe for numerous reasons. Dendritic spines are quite small; they typically measure between $0.2 \mu \mathrm{m}$ to $2 \mu \mathrm{m}$ in length and

\footnotetext{
XIII Activated excitatory synapses make it more likely that a neuron will produce an action potential, whereas activated inhibitory synapses do the opposite.
} 
can measure $\leq 40 \mathrm{~nm}$ at their thinnest spot. Because of the small dimensions of dendritic spines, even subtle changes can potentially lead to functional changes. To observe such subtleties we require good spatial resolution, since distorted or blurry images reveal few details. A good time resolution would also be beneficial if we want to observe the changes as they happen and if we are interested in whether they are long-lasting functional changes or merely temporary fluctuations, and whether they occur as response to certain stimuli of situations. Changes can occur over seconds, minutes or hours and, of course, can last for a few minutes (the phone number you are trying to remember) or an entire lifetime (your parents' voices). This requires the capacity to observe fast changes as well as to monitor these changes continuously over hours or repeatedly over days, months or years without causing damage to the brain. If we intend to observe memory(-like) processes in action, then there is no real point in observing isolated neurons in vitro. Instead we should examine neurons that are still integrated in a functional neural network and can emit and receive signals from other brain cells. This requires that neurons must be embedded in neural tissue and surrounded by other functional neurons. Unfortunately, neural tissue is a fairly dense, inhomogeneous material that causes light scattering and beam aberrations, thereby distorting any signal passing through. Such obstacles would need to be overcome or at least alleviated to enable detailed examinations. A further consideration is the region of the brain in which we would like to observe the changes. If we leave the brain intact, then we can access the outer layers of the cerebral cortex, but this bars us from investigating regions that are embedded deeper inside the brain, such as the hippocampus. Yet contemporary research suggests that the hippocampal formation plays a pivotal role in memory formation, possibly acting as mediator and processor that collects short-term memories, interconnects them with various other memories and then sends them off to be stored in various locations in the brain. A method to examine hippocampal neurons without completely destroying the functional network entails cutting ( $300 \mathrm{~nm}$ to $400 \mathrm{~nm}$ ) thick hippocampal slices and cultivating them under appropriate conditions. Hippocampal brain slices can be sustained over weeks and even months in this fashion, while preserving large parts of the synaptic network and the natural consistency of the brain tissue. 


\subsection{Specific aims for this dissertation}

Can nanoscopy satisfy this wide range of demands and can it contribute substantially to progress in this field of neuroscience? In this dissertation we find solutions for a number of the technical problems, using a multi-facetted approach to imaging synaptic plasticity in an intact physiological environment. We also provide examples of nanoscopy being able to answer scientific questions other methods have not been able to solve. Using STED and RESOLFT nanoscopy we demonstrate the high detail in which dendritic and postsynaptic structures can be imaged. We elaborate how the depth penetration range of the STED and RESOLFT nanoscopes can be improved using simple aberration-correction techniques. As proof-of-principle we measure the width of spine necks throughout cultured hippocampal brain slices in depths of up to $120 \mu \mathrm{m}$, which represented the maximal thickness of the brain slices. By compensating the sample-induced aberrations, we can preserve the spatial resolution of $60 \mathrm{~nm}$ even in great depths up to $\geq 90 \mu \mathrm{m}$. We examine the morphology and motility of dendritic spines, and observe how they fluctuate and change over time. Simultaneously the distribution of the cytoskeletal protein actin is monitored, a protein thought crucial in governing spine plasticity.

Furthermore we examine the morphological response of dendritic spine necks to long-term stimulation procedures, which are the basis of Long-Term Potentiation ${ }^{50,51}$ (called LTP). LTP is a key phenomenon closely linked to memory formation and results in a heightened synaptic response following potentiating pulses of electrical stimuli that may last for hours or even longer. With the spatial resolution of STED and RESOLFT nanoscopy, the width fluctuations and changes of the dendritic spine necks, which can be much smaller than the diffraction limit, can be observed in high detail. By using RESOLFT nanoscopy with reversibly switchable fluorescent proteins, we can image brain structures in nanoscopic detail while using six orders of magnitude less light than with STED nanoscopy or two-photon techniques. These low light levels help to alleviate concerns over whether or not the imaging procedure itself might influence the sensitive synaptic structures, allowing us to noninvasively examine natural dynamic processes. Finally, we discuss the implemented methods and techniques, suggest improvements and analyze the presented data. In conclusion, we present nanoscopy techniques that enable the 
observation of hitherto unobservable dynamic processes in the living brain and thereby offer the potential of unraveling some of the mysteries behind memory formation in the brain. 


\section{Materials and methods}

This chapter describes all the equipment, material and methods utilized in this thesis. The goal of this dissertation was to enable the use of superresolution microscopy deep inside living neuronal tissue for visualizing and observing the dynamic processes that make our brains work. First, we discuss possible brain preparations and samples, finally settling on the use of cultivated brain slices from mouse hippocampi. The preparation and cultivation methods are described and the tools for them listed. Next, we consider the optical microscopy methods that were used for the neuronal imaging experiments. The setups for both the STED and RESOLFT microscopes are explained in detail, along with the further equipment used in the experiments. In brief some of the important parameters of the experiments are detailed. Finally, we consider the labeling method used to visualize the neuronal structures using fluorescence microscopy. The actinbinding label Lifeact is detailed and its characteristics described. The different types of fluorescent proteins employed in the experiments are listed and explained. We discuss the viral transduction method with which the neurons are coerced to express the desired fluorescent label using their own cellular machinery. Finally we describe the chemical agents employed in these experiments, which were used to elicit certain reactions from the observed brain cells. 


\subsection{Organotypic hippocampal brain slices}

In this thesis we examined neurons in cultured hippocampal brain slices. The physiology of these brain slices closely resembles that of natural brain tissue, wherefore they are also called organotypic. ${ }^{52}$ Brain slices, however, are more challenging to image than isolated neurons in the commonly used dissociated tissue cultures. These dissociated cultures are created by removing a living brain and then dissolving the very structure of the brain in several steps, causing the individual brain cells to disentangle and separate. These cells are then spread out on a coated coverslip, onto which they reattach and form new connections. In this process, naturally, the entire neuronal network of interconnecting brain cells is destroyed, only to be replaced by a crude set of newly-formed connections. Obviously, the actual network of the intact brain can only be examined if the interconnections between individual neurons are left (mostly) intact. Ideally this means examining the brain of a living animal in vivo, which is the most physiologically relevant system possible. This is no simple feat, though, because imaging in vivo is complicated by a multitude of additional problems. Unaddressed, these complications prohibit imaging entirely; for instance, any bodily motion must be suppressed or compensated for. Recently, in vivo nanoscopy has been demonstrated in the upper fringes of the cerebral cortex of an anaesthetized mouse. ${ }^{53}$ Nevertheless, this approach was limited to imaging neurons within about $15 \mu \mathrm{m}$ of the brain surface, thereby restricting the observable cells to a very small subset inside the brain. Regions such as the hippocampus, which is embedded deep within the brain, cannot be reached at all by this method.

Brain slices are an excellent compromise between maintaining the interconnectivity of the brain, simplifying the imaging procedure and gaining access to new brain areas. In principle, there are two different sorts of brain slices: acute (freshly cut) and organotypic (cultured). In general, acute slices are used very quickly after being cut from the living brain, and are maintained only for a short period of time, i.e. several hours, before they deteriorate and the slice must be discarded. In contrast, organotypic slices are not used straight away but instead cultured for several weeks before being used for experiments. They can be maintained over extended periods of time, and are generally imaged within $2-8$ weeks in vitro. It can be argued that 
acute slices mirror the physiology of the brain more accurately, as they are actual sections of the adult brain (or from whatever stage of development desired), and have not been altered during the cultivation period. On the other hand, the cells close to the brain slice surface will have been damaged severely, with cells further inside also possibly impacted due to truncated axons or dendrites. In an acute slice, this damage cannot be repaired and results in a layer of dead or dying cells at the sample interface, creating a layer of dense, scattering, opaque tissue with a jagged interface, which complicates imaging profoundly. Even though this layer of dead cells is initially also present in organotypic brain slices, it disappears as the brain slices are given time to recuperate. Over the next couple of days, damaged cells in the cultured slices can be disassembled and catabolized using the machinery inherent to the functioning brain. The remaining healthy cells adapt to their new environment and form a smooth interface on the coverslip, which is beneficial to imaging. Also, neurons that suffered from truncated appendages can regrow new ones and form new interconnections in the brain. In fact, it has been shown that the development of brain slices in vitro mimic the development in vivo to an astonishing degree. ${ }^{54}$ Another benefit from the longevity of organotypic slices is the possibility of transfecting the brain slices using various methods, and being able to maintain them in culture until the specific label is expressed. In addition, the slices can be imaged for extensive periods without the health of the neurons in the slice degrading perpetually over time. Consequently, organotypic brain slices constitute a wellsuited system for examining the function and structure of the living brain with optical methods.

\subsubsection{Brain slice preparation}

The organotypic brain slices were prepared according to the roller-incubator method. ${ }^{52}$ In this procedure, hippocampal brain slices are cut and then fixed onto a glass coverslip by embedding them in a drop of rapidly polymerizing chicken plasma. The slices can then be sustained for months in small tubes inside a 'roller incubator', which periodically immerses into and then removes the slices from a nourishing medium. The preparation of brain slices adhered strictly to the ethics guidelines prescribed in the German and European Animal Welfare Laws, and was approved by the animal welfare officer of the MPI-bpc. Postnatal 5-7 day old mice were 


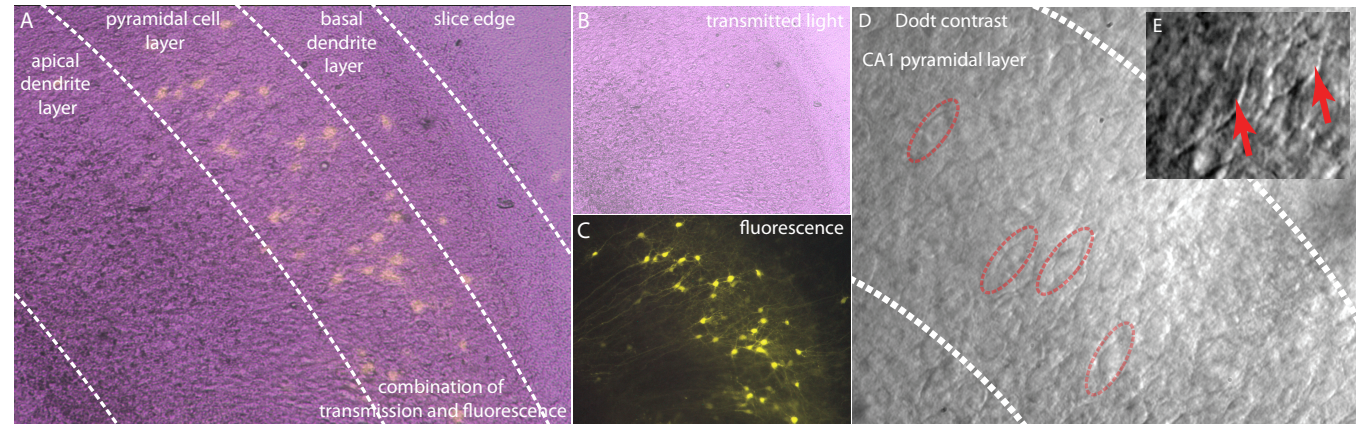

Figure 2.1. I CA1 pyramidal neurons are arranged in a tight cell band in the hippocampus. (A) A combined fluorescence and transmitted light image of the CA1 region in the hippocampus. The cell bodies of labeled CA1 pyramidal neurons are located in a tight band. (B) Transmitted light image of the area in (A). Without enhanced contrast it is difficult to discriminate individual neurons. (C) Fluorescence widefield image of the area in (A). The long apical dendrites of CA1 pyramidal neurons project in one direction and the shorter, branched basal dendrites in the other. (D) By enhancing the widefield image contrast with Köhler illumination and a Dodt-Gradient-Contrast individual neurons can be readily distinguished (some examples circled in red). (E) Zoom-in of an area close to (D) with tightly-packed cell bodies.

decapitated, the brain removed and kept in chilled preparation solution during dissection. Then, the hippocampi were removed and transferred to the tissue chopper, which cut the hippocampus perpendicular to the septotemporal axis (thus creating transverse sections) into $350 \mu \mathrm{m}$ to $400 \mu \mathrm{m}$ thick slices. The time in which the brain slice is removed from the chilled solution must be kept as short as possible to prevent the slice from deteriorating. Back in the chilled preparation solution any remainders of the cortex were removed from the hippocampus slices, which were then left for 30-90 minutes in chilled preparation solution. After that period, individual slices were transferred and glued to the coverslips by sliding them into a drop of chicken plasma, which was then mixed with an equal amount of a clotinducing thrombin solution. The drops were allowed to slowly polymerize; the coverslips were transferred into delta-tubes as soon as the plasma clot had solidified enough to secure the slice to the coverslip. The delta-tubes were filled with just enough culture medium, so that, when kept in the roller-incubator, they were either completely immersed in medium or completely outside the medium, depending on the momentary angle inside the roller-incubator. The slices were maintained at $35^{\circ} \mathrm{C}$ in the roller incubator, which rotated at $10-12$ revolutions/hour. The slices 
were left for 3-4 days in the incubator, and then the culture medium was doped with $10 \mu \mathrm{l}$ of an antimitotic solution, which was washed out after 16-24 hours. Then, the slices were allowed to mature for a total of 12-14 days in vitro before they were used in the experiments. Slices were used in a time frame of 2-8 weeks in vitro.

The culture medium (200ml) contained: $97 \mathrm{ml}$ Basal Medium Eagle (BME), 50ml Hank's Balanced Salt Solution (HBSS), 50ml Horse Serum, 55.5mM D-Glucose ( $2 \mathrm{ml}$ solution) and $1 \mathrm{mM} \mathrm{L-Glutamine}(1 \mathrm{ml}$ solution); the medium was sterile filtered before use. The antimitotic solution contained (in $\mathrm{mM}$ ): Uridin 5, Ara-C hydrochloride 5, 5-Fluoro-2'-deoxyuridin 5 and was sterile filtered before use.

\subsection{Optical microscopy setups}

A microscope suitable for imaging live neurons needed to fulfill certain requirements for the brain cells to be kept alive and functioning throughout the entire duration of the experiment. The brain slices used in these experiments needed to be heated and perpetually suffused with fresh artificial cerebrospinal fluid (ACSF), so as to maintain constant temperature, $\mathrm{pH}$, osmolality and solute composition. Furthermore, the microscope needed to be able to image deep inside dense neuronal tissue, so that neurons could be observed in an intact physiological environment. An additional wide-field scheme (with enhanced contrast in transmitted-light mode and separate fluorescence capabilities) was required for visualizing individual neurons and their larger surroundings, so as to identify specific neuron types or brain regions and put them into anatomical context (Figure 2.1).

To accomplish these ends, two existing superresolution microscopes were modified and adapted for live neuron imaging. The STED microscope, which was used for the depth penetration experiments and for imaging actin dynamics, underwent considerable modification and enhancement. It was fitted with extensive peripheral equipment for labeling, patching, wide-field imaging and slice maintenance. A glycerol immersion objective lens was added to enable deep tissue imaging in brain slices. The RESOLFT microscope was modified for faster imaging and resolution enhancement in all three spatial dimensions, but was otherwise only fitted with the necessary equipment for heating and depth penetration, due to space constraints. 
Both of these setups, along with necessary peripheral equipment, are described in detail in this section.

\subsubsection{A STED setup for imaging living brain slices}

A compact setup for transfecting, examining and imaging brain slices was constructed around an inverse STED microscope (Figure 2.2). The setup featured a commercial Leica DMI6000 B microscope stand, which served multiple purposes. First, it was highly stable while simultaneously offering plenty of space to add peripheral equipment, such as buffer perfusion in- and outlets, sample heating, ground- and stimulation electrodes as well as micromanipulator access. Furthermore, it offered a very generous, extensive wide-field: large regions of a sample could be viewed in stereo using an appropriate ocular, enabling the depth-perception that is crucial for successful micromanipulator interaction. Wide-field images could optionally be observed and recorded using a small, side-mounted camera. The wide-field illumination was enhanced to create higher contrast in an otherwise almost featureless sample. A condenser ( $\mathrm{S} 28, \mathrm{NA}=0.55$, Leica Microsystems) was installed for creating a very even illumination of the sample in a Koehler illumination configuration. The numerical aperture of the condenser head was limited by the corresponding reciprocal working distance and was chosen as the best compromise that still enabled the accommodation of the peripheral equipment, in particular the micromanipulator and the perfusion in- and outlets. The illumination scheme was further enhanced, specifically for viewing thick brain slices, by coupling a DodtGradient-Contrast system (DGC, manual, Luigs \& Neumann, Ratingen, Germany) between the condenser and the lamp housing. This contrast system was developed specifically for use in thick tissue slices. It uses annular illumination to create a gradient contrast along one direction of the sample, which adds phase information to the transmitted light image, thereby helping to visualize low-contrast phase objects such as unstained neurons and dendrites. The direction of the light gradient can be rotated, so as to better visualize structures running in different directions inside a sample. Also, this enabled the use of micropipette techniques, with which transfection or patch-clamping could be performed. 


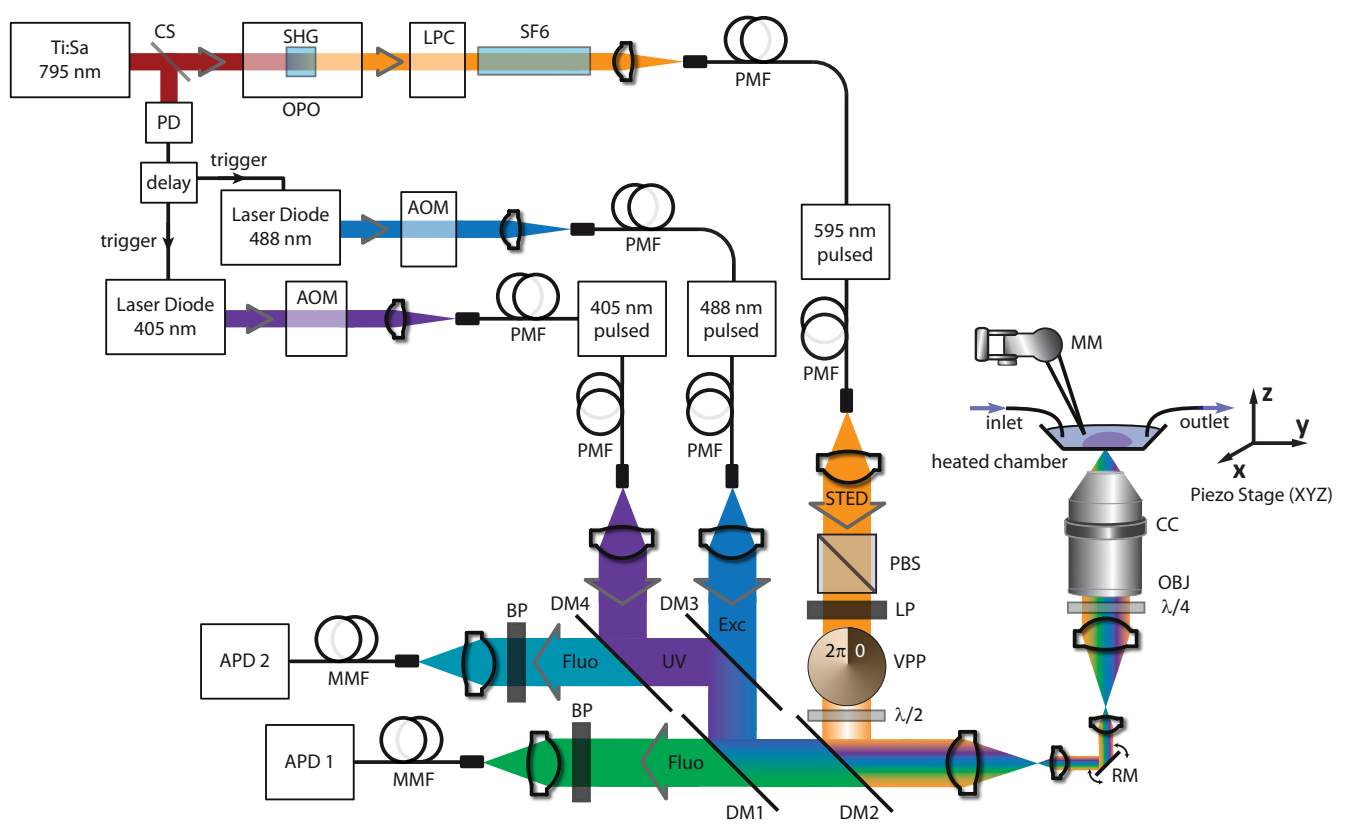

Figure 2.2. | Setup scheme of our custom-built STED microscope for imaging living brain slices, featuring a pulsed $488 \mathrm{~nm}$ pulsed blue laser diode for fluorescent excitation in the main channel, a pulsed $405 \mathrm{~nm}$ pulsed UV laser diode for excitation in the secondary channel and switching of photochromic dyes, and a pulsed $595 \mathrm{~nm}$ de-excitation beam, generated by a Ti:sapphire laser pumping an optical parametric oscillator (OPO). SHG: second harmonic generating crystal; LPC: laser power controller; SF6: $15 \mathrm{~cm}$ SF6 glass rod; CS: glass coverslip; PD: photodiode; AOM: acousto-optic modulator (for switching and intensity control); PBS: polarizing beam splitter; VPP: vortex phase plate generating $x, y-$ donut; LP: longpass laser clean-up filter; DM: dichroic mirror; $\frac{\lambda}{2}, \frac{\lambda}{4}$ : half-wave and quarterwave retarder plates; RM: resonant mirror; OBJ: objective lens; $\mathrm{CC}$ : correction collar; $\mathrm{MM}$ : micro-manipulator; BP: bandpass filter; PMF: singlemode polarization maintaining fiber; MMF: multimode fiber (acting as confocal pinhole); APD: avalanche photodiode;

The STED microscope was optimized for use with fluorescent proteins emitting in the range of $500 \mathrm{~nm}$ to $540 \mathrm{~nm}$, in particular the enhanced green and yellow fluorescent proteins (EGFP and EYFP, respectively). There are a plethora of transgenic animals and cell lines, protein labels and antibodies that employ these fluorescent proteins, all of which could potentially be imaged with this setup. Both EGFP and EYFP can be excited with $488 \mathrm{~nm}$ illumination, which was generated in this setup by a pulsed laser diode (PicoTA 488, PicoQuant, Berlin, Germany) with a pulse length $\approx 100 \mathrm{ps}$ and an average power of $0.5 \mu \mathrm{W}$ to $3.0 \mu \mathrm{W}$ in the objective aperture. This excitation beam could be controlled using an acousto- 
optic modulator (MTS110-A3-VIS, AA Opto-Electronic, Orsay, France), enabling $\mu s^{-}$ fast switching and intensity regulation. A secondary, confocal excitation laser in the UV range $\left(405 \mathrm{~nm}, \mathrm{p}_{\text {avg }} \approx 6 \mu \mathrm{W}\right.$ to $22 \mu \mathrm{W}$ in the aperture, pulsed laser diode, LDH-P-C-405B, PicoQuant) could be used with dyes such as the enhanced cyan fluorescent protein (ECFP) or Atto 390, or for switching certain photochromic dyes (as used in RESOLFT experiments) such as Dronpa and rsEGFP. This laser was also controlled using an acousto-optic modulator (MTS130-A3.400.442, AA OptoElectronic). The de-excitation beam for the main STED channel was optimized for EYFP by selecting a wavelength of $595 \mathrm{~nm}$. This beam was generated by passing the $795 \mathrm{~nm}$ infrared light of a pulsed, mode-locked titanium-sapphire (Ti:Sapphire) laser (MaiTai, 80 MHz, Spectra-Physics, Darmstadt, Germany) through an optical parametric oscillator (OPO, APE, Berlin, Germany). The OPO converts the wavelength of the originally infrared laser light when the photons traverse the integrated optical resonator and second-harmonic generating crystal (SHG), thereby allowing the wavelength output beam to be adjusted over a wide spectral range. This flexibility enables the de-excitation beam to be optimized for various fluorescent dyes, as the optimal de-excitation wavelength varies depending on the utilized fluorescent dye. The de-excitation laser power could be controlled remotely using a laser power controller (LPC, Cambridge Research and Instrumentation, Cambridge, MA, USA) located after the OPO. The original pulses emitted from the Ti:Sapphire laser were very short, only $200 \mathrm{fs}$ in width, and therefore needed to be stretched. Very high pulse peak powers can cause damage to glass fibers, resulting in them becoming increasingly opaque. Stretching also improves the de-excitation interaction with the fluorescent dye molecules in the focal plane, as well as preventing the necessary high peak powers that would lead to two-photon excitation. Therefore, in a first step, the pulses were stretched to picosecond length by passing them through a $15 \mathrm{~cm}$ glass rod (home-built) made of specialized, highlydispersive SF6 glass. The pulses were stretched further by traversing a 50m long, polarization maintaining fiber (OZ Optics, Ottawa, Canada), to a resulting pulse length of $\approx 300 \mathrm{ps}$.

On the optical table, all three laser beams need to be aligned co-axially, and the STED donut imprinted onto the de-excitation beam. All laser beams were coupled into the setup on the optical table using single-mode, polarization maintaining 
fibers. After the fiber, the $595 \mathrm{~nm}$ de-excitation beam was first passed through a polarizing beam splitter (PTW5, $450 \mathrm{~nm}$ to $650 \mathrm{~nm}$, Bernhard Halle Nachfolger $\mathrm{GmbH}$ (B.Halle), Berlin, Germany), ensuring a fixed polarization angle, and then through a longpass cleanup filter, before passing through the vortex phase plate (VPP-1, RPC Photonics, Rochester, NY, USA). This polymeric phase plate imprints a helical phase pattern onto the incoming wavefront, which generates the STED donut in the focal plane of the objective lens. After the phase plate the polarization angle of the STED beam was rotated using a $\frac{\lambda}{2}$-plate (B.Halle). The three laser beams were then combined co-axially using two dichroic mirrors, before the beam diameter was reduced using a Keplerian telescope arrangement, so the beams fit nicely onto the small mirror of the resonating beam scanner (SC-30, EOPC, Glendale, NY, USA). This resonating mirror scanned the beam with a frequency of $15 \mathrm{kHz}$ along the (fast) $\mathrm{x}$-axis of the sample. Scanning along the (slow) $y$ - and $\mathrm{z}$-axes was performed by moving the sample using a piezo stage scanner (P-733, Physik Instrumente, Karlsruhe, Germany). The beams entered the microscope stand through a set of specialized tube lenses (Leica Microsystems, Wetzlar, Germany) before passing the $\frac{\lambda}{4}$-plate (B.Halle), which was located immediately before the objective lens, and converted the beams linear polarization to a circular polarization. Finally, the beams were focused) onto the sample by a high-NA glycerol immersion objective lens (PL APO, GLYC CORR CS $21^{\circ} \mathrm{C}, 63 x, N A=1.3$, Leica Microsystems). The resulting fluorescent photons were collected by the same objective lens and traversed the same beam path in reverse, before being separated from the laser light by a dichroic mirror. The green/yellow fluorescence photons from the main color channel were focused through a 535/50 bandpass filter into a multimode glass fiber, and counted using an avalanche photodiode (PerkinElmer, Waltham, MA, USA). The core diameter of the multimode fiber corresponded to 1.47 of the back-projected Airy disc, meaning it functioned as a confocal pinhole. The blue fluorescence light generated by the secondary, UV excitation channel could be detected here or in a separate detection channel, using a second set of dichroic mirror, bandpass filter and APD.

The immersion objective lens used in this setup was designed to use a glycerol/water (80:20) mixture (type G, Leica Microsystems) as immersion medium, which has a refractive index of $\mathrm{n}=1.45$ at room temperature $\left(21^{\circ} \mathrm{C}\right)$ and $\mathrm{n}=1.46$ at $35^{\circ} \mathrm{C}$. This 
specific objective lens has a correction collar, which can be used to negate spherical aberrations occurring in the sample. The objective was pre-corrected for coverslips of $0.14 \mathrm{~mm}$ to $0.18 \mathrm{~mm}$ thickness and has a working distance of $280 \mu \mathrm{m}$. Any minor deviations in temperature, cover slip thickness or refractive index of the immersion medium or sample can be compensated using the correction collar. If, therefore, we keep all other parameters constant, we can correct for aberrations caused by sample refractive indices deviating from $n=1.45$. In organotypic hippocampal brain slices this worked very well for penetration depths less than $50 \mu \mathrm{m}$, at which the correction collar reached its limits. To be able to correct aberrations in even greater depths, we induced artificial spherical aberrations by using an immersion medium with a higher refractive index of $n=1.46$ (series A, Carqille, Cedar Grove, NJ, USA). This expanded the range of the correction capabilities using the collar to depths of up to $\approx 90 \mu \mathrm{m}$. For measurements in depths up to $50 \mu \mathrm{m}$, therefore, we used the intended glycerol/water mixture, and the $n=1.46$ refractive index solution in depths beyond that $(>50 \mu \mathrm{m})$.

In order to perform long-term experiments on brain slices, the setup was equipped to maintain brain slices over long time periods, typically up to 10 hours. To this end, the coverslips containing the brain slices were glued into custom-built sample chambers using a non-toxic, two-component dentist glue (Twinsil Extrahart 32, Picodent, Wipperfürth, Germany). Special care was taken to minimize any coverslip tilt, so as to avoid potentially severe aberrations. The living brain slices were suffused continuously with carbogenated artificial cerebrospinal fluid (ACSF) at a rate of $\approx$ $1 \mathrm{ml} / \mathrm{min}$ using a peristaltic perfusion pump (Minipuls 3, Gilson Inc., Middleton, WI, USA). The tubing and rotation speed of the pump, as well as the fluid in- and outlets were adjusted so as to avoid any bubbles, ripples or waves in the sample chamber, producing a fine smooth and homogeneous flow of medium across the brain sample. Chemical drugs could be applied easily this way without disturbing the setup, simply by switching between several solution reservoirs. To sustain physiological temperatures the sample chamber and the objective lens could be heated separately to $35^{\circ} \mathrm{C}$ throughout the duration of the experiments. 


\subsection{A RESOLFT microscope for imaging neurons with low light levels}

A second setup for imaging living brain slices was constructed around an inverse RESOLFT microscope developed for imaging reversibly switchable fluorescent proteins (RSFPs). This RESOLFT setup (Figure 2.3) was also modified to sustain living brain slices over the course of the experiments by adding heating capabilities, similar to the STED setup. Due to spatial constraints, though, this setup needed to be constructed more compactly. Therefore, all excess peripheral equipment that could be spared was omitted, and all secondary sample preparation or pre-analysis tasks were outsourced to the modified STED setup. The perfusion system also needed to be omitted for stability reasons; a HEPES buffered ACSF solution was favored over the previously used carbonate buffered ACSF, as it did not require any carbogen bubbling to maintain physiological $\mathrm{pH}$-levels.

The RESOLFT setup was modified further, so as to enable fast imaging of neuronal dynamics deep within thick brain slices. First, the pulse and switching scheme of the RSFPs was optimized for Dronpa-M159T, a fast-switching variant of the wellknown RSFP Dronpa. This enabled considerably faster imaging speeds than in previous RESOLFT implementations, ${ }^{55,56}$ capable of recording dynamics on even a sub-second scale. Second, a glycerol objective lens was implemented, enabling deep tissue imaging. Finally, a second de-excitation beam was added to the setup, adding the capabilities for imaging with improved resolution in all three spatial dimensions.

The RESOLFT setup offered two distinct advantages over the STED setup. First of all, by using a different method for switching the fluorescence on and off in a targeted manner - using RSFPs - a RESOLFT microscope requires much less illumination for switching, resulting in $10^{5}-10^{6}$ times lower light levels than necessary for STED microscopy. Obviously, this greatly reduced the amount of light with which neurons were irradiated during imaging experiments, consequently reducing the possibilities of photo-induced effects in the sample that might impact the observed behavior of otherwise undisturbed brain cells. A small drawback of this switching method was that near-UV illumination was required for the on-switching of the RSFPs. This is usually avoided when imaging live cells, due to the potential cell damage that UV 


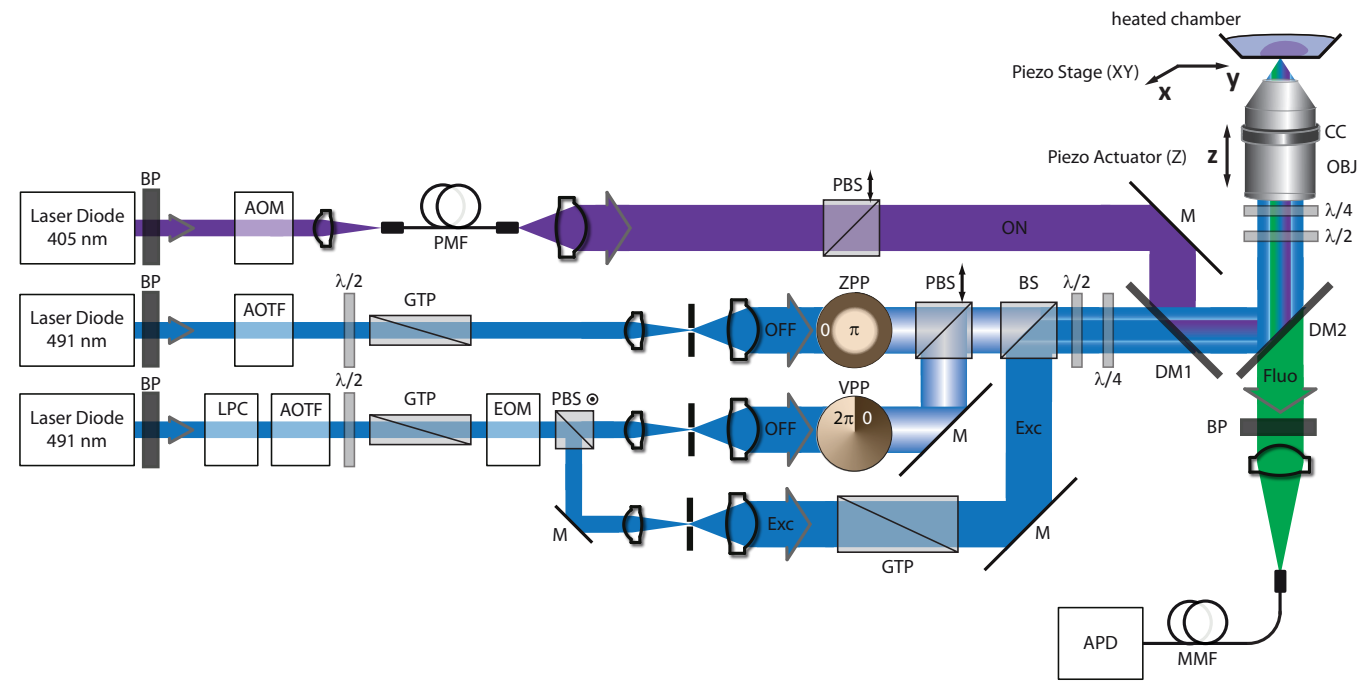

Figure 2.3. | Setup scheme of our home-made RESOLFT microscope, featuring a $405 \mathrm{~nm}, 30 \mathrm{~mW}$ UV-laser for on-switching and two $491 \mathrm{~nm}$ blue laser with $10 \mathrm{~mW}$ and $50 \mathrm{~mW}$ for off-switching and read-out. LPC: laser power controller; AOM: acoustooptic modulator; AOTF: acousto-optic tunable filter; $\frac{\lambda}{2}, \frac{\lambda}{4}$ : half-wave and quarter-wave retarder plates; GTP: Glan-Thompson Prism; EOM: electro-optic modulator (for polarization rotation); PBS: polarizing beam splitter with annotated polarization orientation; 40, 100, 300: doublet lenses of respective focal length; $\mathrm{PH}: 10 \mu \mathrm{m}$ pinhole; VPP: vortex phase plate for $\mathrm{x}, \mathrm{y}$-donut; ZPP: $0-\pi$ phase plate for $\mathrm{z}$-donut; BS: 50:50 beam splitter; $\mathrm{M}$ : dielectric mirror; DM1,2: dichroic mirrors; BP: bandpass filter; CF: laser clean-up filter; OBJ: objective lens; CC: correction collar; APD: avalanche photodiode; PMF: singlemode polarization maintaining fiber; MMF: multimode fiber (acting as confocal pinhole). This RESOLFT setup was constructed and modified by llaria Testa.

radiation can cause. This imaging scheme required very little UV light, however, due to the fast switching times of Dronpa-M159T and the already minimal light required for switching in a RESOLFT microscope. The second advantage was the added capability to superresolve structures in three spatial dimensions, instead of only in the optical $(x, y)$ plane. The resolution enhancement along the optical $(z)$ axis was made possible by adding a second de-excitation beam with a different phase pattern imprinted on the wavefront. This second beam was passed through a special $(0-\pi)$ phase plate that imprinted a phase pattern to create a ' $z$-donut' onto the wavefront, which was designed to switch off fluorophores along the optical axis. By adding this second de-excitation beam to the first we were able to restrict the fluorescence both in the optical plane and along the optical axis, creating a three- 
dimensional de-excitation donut ${ }^{57}$. Both beams were adjusted to have the same average laser power, leading to a near isotropic resolution increase. The absolute resolution in the $x, y$-plane was still superior to the resolution along the $z$-axis by approximately a factor of two, because of the inherent higher spatial resolution in the optical plane in a $2 \pi$-configuration.

To implement the RESOLFT switching scheme this microscope required two different laser wavelengths and a total of four separate, co-axially aligned beams: one UV beam for on-switching, two blue beams for de-excitation and one blue beam for fluorescent read-out. The first beam, featuring a wavelength of $405 \mathrm{~nm}$ and a Gaussian beam profile, was generated by a continuous wave (CW) diode-pumped solid-state (DPSS) laser (CL-405-030-S, $30 \mathrm{~mW}$, CrystaLaser, Reno, NV, USA) and was used for activating the RSFPs, enabling them to be excited to a fluorescent state. The two de-excitation beams were generated using two separate, but identical CW lasers (Calypso 50, DPSS, $50 \mathrm{~mW}$, Cobolt, Stockholm, Sweden) operating at $491 \mathrm{~nm}$. By using two non-coherent light sources we avoided any interference phenomena within the focal volume. Each of these beams was passed through one of two phase plates, thereby generating either an $\mathrm{x}, \mathrm{y}-(463 \mathrm{~nm}$ mask, vortex plate VPP-A, RPC Photonics, Rochester, NY) or a z-donut ( $0-\pi$ phase mask, home-built). A fourth beam was created by diverting some light from one of the $491 \mathrm{~nm}$ lasers. This beam bypassed the phase plates and therefore maintained a Gaussian beam profile; this $491 \mathrm{~nm}$ beam was used for fluorescent excitation of the activated RSFPs. The pulse cycling sequence, i.e. duration and order of the various pulses, was generated using a pulse generator (Model 9514, QUANTUM COMPOSERS, Bozeman, MT, USA) and controlled using a fast acquisition card (MCA-3 Series / P7882, FAST ComTec $\mathrm{GmbH}$, Oberhaching, Germany). To enable the fast beam-switching times we used electro- and acousto-optic devices; acousto-optic modulators (MTS 130A3, Pegasus Optik GmbH, Wallenhorst, Germany) or acousto-optic tunable filters (AOTF.nC/TN, Pegasus Optik $\mathrm{GmbH}$ ) were used for switching beams on and off, whereas an electro-optic modulator (LM 0202, 5W, 400-650nm, with pulse amplifier LIV 20, Linos, Göttingen, Germany) was used to switch between the $x, y$-de-excitation beam and the read-out beam, by rotating the beams' polarization by $90^{\circ}$. 
As with the STED setup, we utilized two different objective lenses with this setup. A high-NA oil immersion objective lens (HCX PC APO, 100x, NA=1.4; Leica Microsystems, Wetzlar, Germany) was used for imaging structures in depths up to $10 \mu \mathrm{m}$. Generally, we used a glycerol immersion objective lens (PL APO, GLYC CORR $\mathrm{CS} 21^{\circ} \mathrm{C}, 63 \mathrm{x}, \mathrm{NA}=1.3$; Leica Microsystems), enabling deeper penetration within the living brain samples, between $5 \mu \mathrm{m}$ to $50 \mu \mathrm{m}$. The microscope featured a pure stagescanning setup for rastering the beams over the sample, comprising two separate piezoelectric components: a piezo stage was used for translating the sample in the optical $(x, y)$ plane (NV40, Piezosystem Jena, Jena, Germany), whereas a onedimensional piezo-motor was used to translate the objective lens along the optical (z-) axis in a range of $120 \mu \mathrm{m}$ (ENV40/20, Piezosystem Jena). The fluorescent photons were detected using an avalanche photodiode (APD, Perkin Elmer, Waltham, MA, USA), but only when the $491 \mathrm{~nm}$ read-out beam was active. The fluorescence was filtered using a $(532 / 70 \mathrm{~nm})$ bandpass filter.

\subsubsection{RESOLFT switching scheme}

For each recorded pixel in a RESOLFT image the RSFPs in the focal area need to be first switched on, then switched off in the focal periphery and finally excited and read-out, in that order. Consequently, the various laser beams need to be cycled on and off for various durations for each individual pixel. For each RSFP the individual beam timings need to be adjusted to match the individual switching and fluorescence characteristics of the RSFPs. For Dronpa-M159T the following switching scheme proved to be the most efficient for fast imaging of neuronal dynamics. First, the RSFPs were switched on using a $40 \mu \mathrm{s}$ short illumination pulse with $405 \mathrm{~nm}$ ultraviolet light (intensity $\mathrm{I}_{O N} \approx 1 \mathrm{~kW} / \mathrm{cm}^{2}$, average power $\mathrm{p}_{\text {avg }}=0.5 \mu \mathrm{W}$ ). Next, the RSFPs on the periphery of the focal volume were switched into the off-state using an extended $400 \mu$ s dose of $491 \mathrm{~nm}$ blue light $\left(\mathrm{I}_{O F F} \approx\right.$ $\left.1.2 \mathrm{~kW} / \mathrm{cm}^{2}, \mathrm{p}_{\text {avg }}=3 \mu \mathrm{W}\right)$. The RSFPs could be switched off in high resolution in the optical plane by using a single de-excitation beam with an $x, y$-donut. In addition, RSFPs could be switched off in high-resolution along the optical axis by adding a second de-excitation beam with a $z$-donut. In the latter case the total illumination intensity during de-excitation was equal to the sum of both beam intensities, so $2 \times I_{O F F}$. During the final step, any molecules remaining in the fluorescent on-state 
in the center of the focal spot were excited using a $40 \mu$ s pulse of $491 \mathrm{~nm}$ blue light (Gaussian beam profile, $\mathrm{I}_{e x c} \approx 3.8 \mathrm{~kW} / \mathrm{cm}^{2}, \mathrm{p}_{\text {avg }}=3 \mu \mathrm{W}$ ). Only during this last step was the APD permitted to record incoming photons. This illumination pattern was conducted pixel by pixel throughout the entire image.

\subsection{Labeling method, fluorescent proteins and viral transduction}

\subsubsection{Lifeact: an actin-binding peptide}

For the majority of experiments we used the actin-binding peptide Lifeact for labeling the neurons in the hippocampal slices. Lifeact is a 17-amino-acid long peptide that was isolated from a significantly larger actin-marking protein found in yeast after the discovery that these 17 amino acids were entirely sufficient for actin localization. The small size of Lifeact and the weak-binding affinity ensures that it does not interfere in regular cellular actin dynamics and does not alter the natural actin filaments in cells. ${ }^{58}$ Lifeact binds to both monomeric actin (globular or G-Actin) and filamentous actin (F-Actin). The binding affinity of Lifeact is 10-30 times higher for G-Actin $\left(\mathrm{K}_{d}=40 \mathrm{nM}\right.$ to $\left.280 \mathrm{nM}\right)$ than for F-Actin $\left(\mathrm{K}_{d}=1.3 \mu \mathrm{M}\right.$ to $\left.2.3 \mu \mathrm{M}\right)$. Given the chance, Lifeact is therefore more likely to bind to free actin than to filamentous actin. This means that in transfected neurons there will be constant background of Lifeact bound to diffusing actin monomers in the cytosol, providing a weak volume labeling of the neuronal morphology. Actin filaments, however, will be considerably brighter than this background due to the higher concentration of actin molecules within the filaments. The low binding affinity to F-Actin has special benefits when repeatedly imaging actin-filaments. Lifeact does not fuse permanently to actin, unlike other common actin labeling techniques such as phalloidin or actin that was fused to a fluorescent protein by genetic modification; moreover, Lifeact binds and unbinds repeatedly due to its low binding affinity to actin. Therefore, fluorescent Lifeact-labels that were bleached during previous imaging can unbind from an actin filament and be replaced by a fully functional fluorescent protein. Because the imaging volume in our experiments was always far smaller than the entire volume of the corresponding neuron, any potentially bleached molecules inside the imaging 
volume were greatly outnumbered by undamaged fluorescent proteins elsewhere in the cell. Given enough time for diffusion or transport in and out the imaged volume, this trait of Lifeact ensures a virtually endless supply of fresh labeling molecules. In long-term experiments over several hours this resulted in near-constant image brightness, especially if enough time between images was allotted for the fluorescent label to completely replenish (typically between $10 \mathrm{~s}$ to $60 \mathrm{~s}$ ).

\subsubsection{Fluorescent markers for STED and RESOLFT}

In order to facilitate the fluorescent microscopy of living cells with minimal invasiveness, we used fluorescent proteins as label of choice throughout the experiments. It is possible to implant the genetic sequence encoding fluorescent proteins into the genome of living cells, forcing them to read-out and reproduce the fluorescent proteins using their endogenous cell machinery. ${ }^{59}$ Because fluorescent proteins are not inherently toxic, and provided that their production inside a cell is not excessive, stable cell lines and even transgenic animals can be created. There is a substantial number of different fluorescent proteins available emitting in the visible and near-visible spectral range. Yet the most prevalent fluorescent label by far that is expressed in stable cell lines, transgenic animals and by genetic transfection, is the green fluorescent protein (GFP) ${ }^{60}$ or its spectrally enhanced variants (e.g. enhanced GFP or: EGFP) ${ }^{61,62}$. Therefore, optimizing the spectral range of the components in fluorescent nanoscopes to include the wavelengths required for GFP is prudent, ensuring the compatibility with numerous biological experiments and a wide array of various labeling tools. In this thesis we used two sorts of fluorescent proteins for labeling, as required by the different microscopy techniques: EYFP, a 'regular' fluorescent protein, was used for most STED experiments, whereas a reversibly switchable fluorescent protein, Dronpa-M159T, ${ }^{63}$ was used for the RESOLFT imaging. Both fluorescent proteins emit in a similar spectral range.

The enhanced yellow fluorescent protein (EYFP) was our fluorescent protein of choice for the STED imaging of living neurons. It boasts a high quantum yield of $\phi_{F L}=0.61$ and a high absorption and is furthermore very resilient to photobleaching. EYFP is a variant of the original GFP with a spectrum shifted towards the yellow (by about $20 \mathrm{~nm}$ ), its excitation maximum at $514 \mathrm{~nm}$ and 
emission peak at $527 \mathrm{~nm}$. The wavelength of EYFP is close enough to that of EGFP that the STED setup could be constructed to allow the imaging of both proteins.

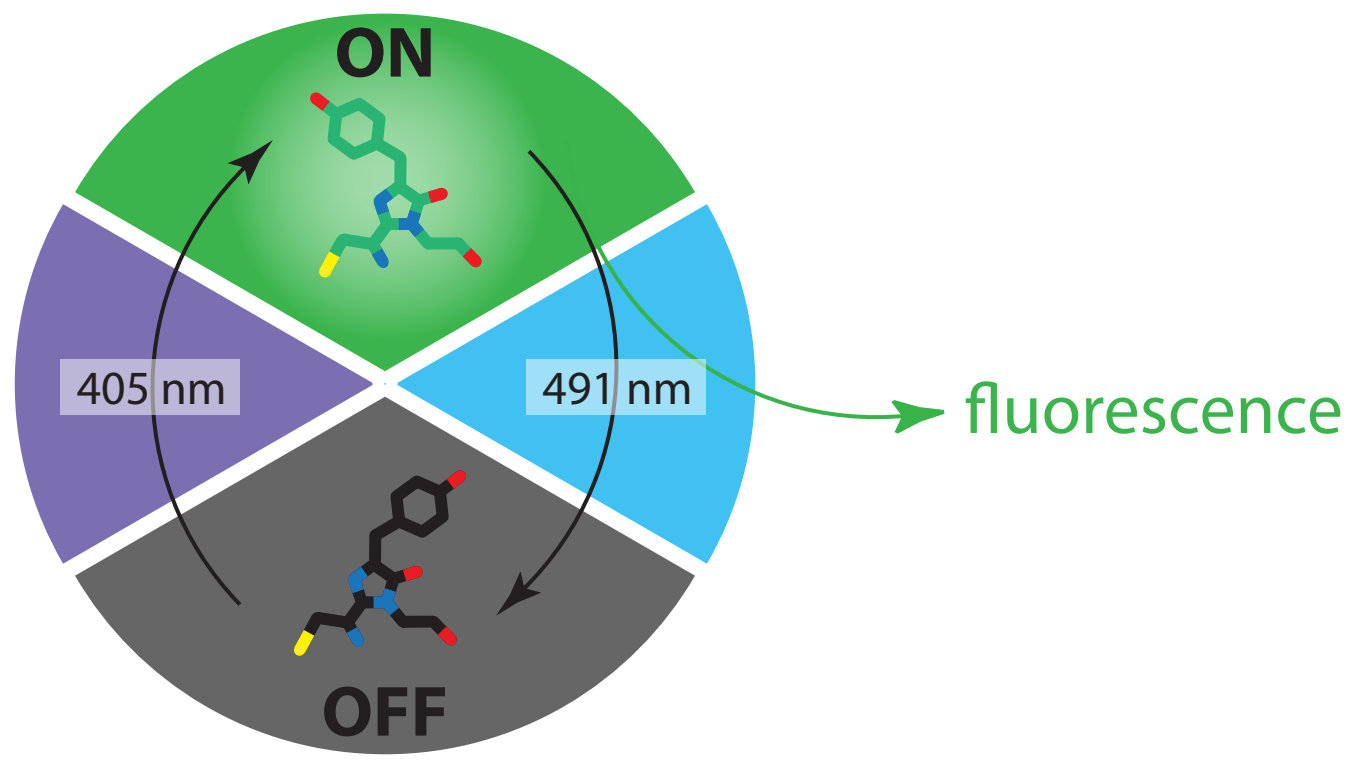

Figure 2.4. | Switching scheme of the reversibly switchable fluorescent protein Dronpa-M159T. Dronpa-M159T can be switched into a long-lived dark-state using $491 \mathrm{~nm}$ light, and back into the stable bright state using $405 \mathrm{~nm}$ illumination, starting the cycle anew. As a negative switcher, Dronpa-M159T can be switched off by the same light used for fluorescent excitation.

\subsubsection{Reversibly switchable fluorescent proteins (RSFPs)}

Certain fluorescent proteins can not only be switched from the ground state into an excited state and back again, but between bright and dark states or between different colors using light (Figure 2.4). This can be either an irreversible process (photoactivation or photoconversion) or a reversible process (photochromism), depending on the protein itself. Obviously, the ability to switch a fluorescent label on and off multiple times is a prerequisite for most fluorescence microscopy methods and for repeated measurements of single molecules. The special subset of fluorescent proteins that are photochromic, i.e. they can be switched reversibly from one chemical state into another using light, concomitant with a change in absorption spectrum, are also referred to as reversibly switchable fluorescent proteins (RSFPs). In short, they change color when exposed to light (of a specific wavelength). The first of these RSFPs was Dronpa, derived from the pectiniidae 
stony coral family, ${ }^{64}$ and asFP595 derived from the sea anemone anemonia sulcata. ${ }^{65}$ Dronpa (and its variant Dronpa-M159T) is a negative-switcher, meaning that it can be both excited and driven to the dark (off-) state using the same wavelength, around $489 \mathrm{~nm}$. It can be recovered from the off-state by brief illumination with ultraviolet $405 \mathrm{~nm}$ light (see Figure 2.4). The underlying chemical process is a light-induced cis-trans isomerization of the chromophore, which occurs concomitant with a great many residue reformations. ${ }^{66}$ The chromophore is in the bright (on-) state when in cis-conformation (deprotonated state) and in the dark (off-) state when in trans-conformation (protonated state). The chromophore moves from a completely different local environment inside the beta-barrel upon cis-trans isomerization, suddenly facing starkly changed electrostatic surface potentials. It seems probable that this would have a strong influence on its protonation state and its ability to fluoresce. Dronpa emits similar to GFP in the green spectral range, with an emission maximum at $522 \mathrm{~nm}$; the emission peak for its variant Dronpa-M159T lies at $515 \mathrm{~nm}$.

Since the development of the first RSFPs there have been significant further developments in this field, creating various different RSFPs with partially superior characteristics that are highly suitable for different tasks. One such variant is Dronpa-M159T ${ }^{63}$, a fast-switching variant of the original Dronpa protein that was used in this thesis for RESOLFT microscopy. Even though the fluorescence quantum yield $\phi_{F L}=0.23$ of Dronpa-M159T is only a quarter of that of the original Dronpa $\left(\phi_{F L}=0.85\right)$, it is very resilient to bleaching and it boasts far superior switching times. The time for switching on Dronpa-M159T $\left(t_{1 / 2}^{o n}=50 \mathrm{~ms}\right)$ is reduced by half, and the time for switching off $\left(t_{1 / 2}^{o f f}=230 \mathrm{~ms}\right)$ is reduced by more than three orders of magnitude. Given a sufficiently bright label, these improved switching times allow dynamic microscopy that is fast enough to observe rapid dynamics of neuronal structures, which can occur on the time scale of seconds or less. In regards to photobleaching, Dronpa-M159T displays a low switching fatigue: it can undergo more than 6000 on-off switching cycles before bleaching by $50 \%$, compared to $<10$ cycles for the original Dronpa. ${ }^{55}$ We used two slightly different variants of Dronpa-M159T in the experiments, namely Dronpa-M159Tv2.0 and Dronpa-M159TGE. The variant Dronpa-M159T-GE is a modification of Dronpa-M159T containing altered $\mathrm{N}$ - and $\mathrm{C}$-termini, and the variant Dronpa-M159Tv2.0 has an additional point mutation E218G. We did not observe a difference between neurons transfected 


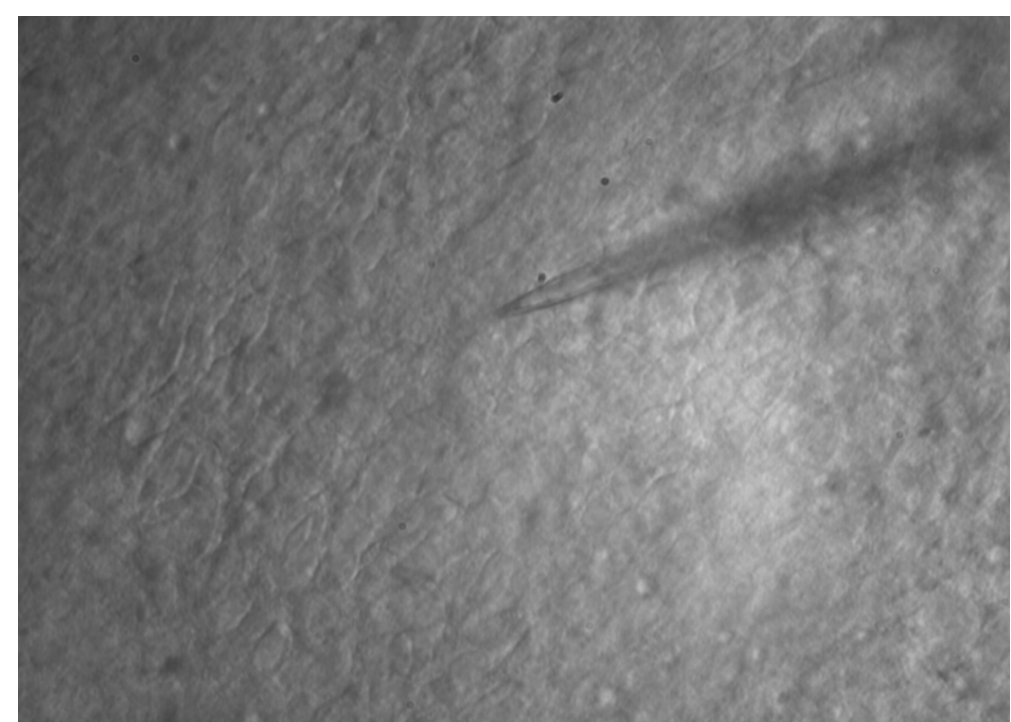

Figure 2.5. | A micropipette injects viral particles into the CA1 pyramidal layer in a hippocampal brain slice.

with Lifeact-Dronpa-M159Tv2.0 and Lifeact-Dronpa-M159T-GE and therefore do not distinguish between these two labels in the manuscript. Other recent RSFP developments include rsEGFP ${ }^{55}$, a deliberate modification of EGFP which offers improved switching times compared to the original Dronpa and very low switching fatigue, sufficient for potential use in data storage, and Dreiklang ${ }^{56}$, a RSFP that can be switched on with one wavelength and read-out with another, offering fully flexible read-out times suitable for imaging dimly labeled structures.

\subsubsection{Viral transduction}

In order for cells to begin producing fluorescent proteins of their own accord, the appropriate genetic sequence must be introduced into the cell's genome. In this thesis this was achieved either by using stable, transgenic mouse lines or by introducing the genetic sequence of the fluorescent proteins and label into brain slices containing unstained neurons of wild-type mouse strains using viral transduction (or transfection). This was accomplished by injecting concentrated doses of a modified virus containing the desired genetic sequence into the intracellular space between neurons in one of the hippocampal cell bands (Figure 2.5). The virus introduces the sequence into the neuron, which begins copying the sequence and producing 
the corresponding fluorescent protein. After being translated the protein needs a certain amount of time to fold correctly before it is able to fluoresce; the specific folding time depends on the fluorescent protein, resulting in different expression times for different fluorescent proteins.

In these experiments we used a modified Semliki Forest Virus that was based on a pSCA3 vector. The virus was specifically deactivated so that a viral particle could transfect one neuron, but not reproduce functional copies of itself and so continue to transfect multiple cells. This reduced its biohazard risk level from S2 status (small risk) to S1 (no risk). The Semliki Forest Virus is an alphavirus that was isolated for the first time from mosquitos captured in the Semliki Forest in Uganda. ${ }^{67}$ The virus targets and replicates specifically in neurons and oligodendrocytes (though not astrocytes), as well as in muscle cells.' We created several variants of the Semliki Forest Virus so that each variant contained the genetic sequence of whatever fluorescent protein and label we required. To create the actin-binding Lifeact label we inserted the coding sequence for Lifeact-EYFP, Lifeact-Dronpa-M159T or LifeactPadron into psCA3; for cytosolic labeling we inserted the sequence for DronpaM159T instead."

For the actual transduction procedure, we loaded several $\mu$ l virus suspension (titer of $10^{5}-10^{8}$ ) into a freshly pulled micropipette and injected each brain slice with several $\mathrm{pL}$, until a visibly large enough spot was formed that just encompassed the cell bodies of a small cluster of neurons in the appropriate cell band (Figure 2.5). The virus suspension needed to be injected precisely into the desired brain regions, which in general were the pyramidal layers of the CA1 and CA3 hippocampal regions and sometimes the granule cell layer of the dentate gyrus. The micropipettes were pulled from thin-walled glass capillaries (borosilicate glass, $1.5 \mathrm{~mm}$ outer diameter; TW150F-4, World Precision Instruments, Sarasota, Florida, USA) using a Narishige model DC-10 micropipette puller (Narishige International Ltd., London, UK), so that they exhibited an opening diameter of a few $\mu \mathrm{m}$, with a typical access resistance in standard artificial cerebrospinal fluid of $\approx 10 \mathrm{M} \Omega$. For transfection, the brain

\footnotetext{
'If a Semliki Forest Virus infection is left unchecked, it can lead to a fatal encephalitis (acute inflammation of the brain); this has been observed in animals, but only once in a human.

"Special thanks to Claudia Huber (MPI of Neurobiology, Martinsried) for the original Lifeact-YFP virus and especially to Katrin Willig for subsequently perfecting, modifying and executing the viral cloning process.
} 
slices were transferred to a sample chamber in the STED-setup, where several $\mathrm{pl}$ of the viral suspension were injected using millisecond long pressurized air pulses delivered by a pressure generator (TooheySpritzer Ile, 14-20 psi, Toohey Company, Farfield, NJ, USA). Afterwards, the slices were replaced in the roller incubator and incubated for at least six hours, typically overnight. The neurons usually expressed the fluorescent marker visibly within six to twelve hours. Transfected brain slices were generally imaged the next day and always within 48 hours of transfection; brain cells that were transfected did not survive much longer than two days.

As mentioned earlier, we also used brain slices from transgenic mouse strains for various purposes. For initial experiments and for control experiments concerning the Lifeact actin-label we used the transgenic mouse strain Thy1-YFP, in which a sparse subset of neurons, mainly projection neurons with the thy 1 gene, expresses YFP endogenously in the cytosol. As CA1 pyramidal cells are projection neurons, hippocampal brain slices from this mouse strain enabled the observation of the morphology of volume labeled CA1 pyramidal neurons. Brain slices from these mice were prepared analogously to wild-type mice.

\subsubsection{Buffers and drugs}

In the course of our experiments we exposed the brain slices to drugs and toxins, either as a test of certain facets of the experiment or for evoking specific responses from the brain cells which then could be studied.

During the experiments the brain slices were sustained in artificial cerebrospinal fluid (ACSF), which was kept at a physiological $\mathrm{pH}$ level of 7.4. The $\mathrm{pH}$ of carbonate buffered ACSF was adjusted by perpetually bubbling the ACSF with carbogen gas $\left(95 \% \mathrm{O}_{2}, 5 \% \mathrm{CO}_{2}\right)$. For ACSF buffered with HEPES the $\mathrm{pH}$ was adjusted to 7.4 using $\mathrm{NaOH}$ prior to the experiment. The standard (carbogenated) ACSF used in this thesis contained (in $\mathrm{mM}$ ): $\mathrm{NaCl} 126, \mathrm{KCl} 2.5, \mathrm{CaCl}_{2} 2.5, \mathrm{MgCl}_{2} 1.3, \mathrm{NaHCO}_{3} 26$, $\mathrm{NaH}_{2} \mathrm{PO}_{4} 1.25$ and glucose 30. The HEPES buffered ACSF contained: $\mathrm{NaCl} 126, \mathrm{KCl}$ 2.5, $\mathrm{CaCl}_{2} 2.5, \mathrm{MgCl}_{2}$ 1.3, HEPES 27 and glucose 30 .

We used a chemical long-term potentiation protocol (chem-LTP) for evoking a broad stimulation of the hippocampal neurons. For this we modified the standard ACSF 
solution by reducing the amount of $\mathrm{Mg}^{2+}$ ions, elevating the amount of $\mathrm{Ca}^{2+}$ ions and adding the chemical tetraethylammonium chloride (TEA-Cl), a drug which blocks potassium channels in the neurons and has been shown to induce longterm potentiation similar in nature to electrically induced LTP. ${ }^{51}$ This potentiation medium was washed into the sample chamber for 7-9 minutes before it was washed out again by the regular ACSF. The modified ACSF for inducing chem-LTP contained (in $\mathrm{mM}$ ): $\mathrm{NaCl} 99, \mathrm{KCl} 5, \mathrm{CaCl}_{2} 5, \mathrm{MgCl}_{2} 0.1, \mathrm{NaHCO}_{3} 26, \mathrm{NaH}_{2} \mathrm{PO}_{4}$ 1.25, glucose 24 and TEA-Cl 25. The solution was carbogenated throughout the experiment to maintain a $\mathrm{pH}$ of 7.4 .

For testing the actin-label and for freezing spontaneous actin dynamics in the neurons we modified the ACSF to contain the drug Latrunculin B. This toxin, which is generated by the red sea sponge latrunculia magnifica, binds to actin monomers, thereby inhibiting actin polymerization and leading to a disassembly of already formed actin filaments. ${ }^{68}$ The functionally identical Latrunculin A molecule does not interfere with Lifeact binding to actin monomers, implying non-overlapping binding sites of both agents on actin. ${ }^{58}$ The addition of Latrunculin into the neurons should therefore not impair the functionality of the Lifeact-YFP labeling. The ACSF designed to induce actin depolymerization contained (in $\mathrm{mM}$ ): $\mathrm{NaCl} 126, \mathrm{KCl} 2.5$, $\mathrm{CaCl}_{2}$ 2.5, $\mathrm{MgCl}_{2} 1.3, \mathrm{NaHCO}_{3} 26, \mathrm{NaH}_{2} \mathrm{PO}_{4}$ 1.25, glucose 30 and Latrunculin $\mathrm{B}$ 0.01 , and was carbogenated throughout the experiment to maintain a $\mathrm{pH}$ of 7.4. For some experiments, in which no response to the suffused Latrunculin B ACSF could be observed, we washed in a slightly different ACSF mixture containing a greater concentration of Latrunculin B. This further modified depolymerization medium contained (in mM): $\mathrm{NaCl} 126, \mathrm{KCl} 2.5, \mathrm{CaCl}_{2} 2.5, \mathrm{MgCl}_{2} 1.3$, glucose 30, HEPES 27 and Latrunculin $\mathrm{B}$ 0.05; the $\mathrm{pH}$ was preadjusted with $\mathrm{NaOH}$ to 7.4. The ACSF for inhibiting actin polymerization was washed in for at least ten minutes before it was washed out again by the regular ACSF. 


\section{Imaging deep inside neuronal tissue by compensating spherical aberrations}

The recent breakthrough in the field of optical nanoscopy has opened up whole new possibilities for neuroscience. For the first time, the development of live neurons can be imaged noninvasively with subdiffraction resolution, revealing dynamic processes such as rapidly moving presynaptic vesicles, ${ }^{69}$ highly plastic morphology of postsynaptic dendritic spines ${ }^{70}$ and the dynamics of single actin molecules in dendritic spines. ${ }^{71}$ So far, however, these superresolving techniques have all been limited to imaging individual neurons in dissociated cell cultures or on the surface of brain slices, unable to penetrate deep inside an intact neural network. The achievable imaging depth inside thick samples is limited by spherical aberrations, scattering and a high fluorescent background occurring inside dense, opaque brain tissue. ${ }^{72}$ The last two can be overcome by two-photon microscopy ${ }^{14}$, which is why only with (diffraction limited) twophoton microscopy brain cells could previously be observed in truly undisturbed physiological environments, such as (acute) brain slices or the brains of live animals. ${ }^{17,73}$ But the desirable subdiffraction resolution has remained elusive, despite recent approaches to enhanced twophoton microscopes by adding a single-photon STED beam, thus improving the two-photon resolution by a factor of three. ${ }^{74,75}$ In this chapter we demonstrate how the addition of simple, yet effective aberration correction capabilities enables a STED nanoscope to penetrate deep inside living brain tissue, while maintaining considerable subdiffraction resolution. The effects of spherical aberrations on acquired confocal and STED images are examined experimentally, allowing an optimal compensation of the otherwise crippling image distortions. This approach enables the extension of tested nanoscopy techniques to previously unreachable depths, as has now been demonstrated by using an identical approach to record in vivo STED images from the depths of the intact brains of living mice. ${ }^{53}$ 
Chapter 3. Imaging deep inside neuronal tissue by compensating spherical aberrations

\subsection{Refractive index of brain tissue}

The refractive index $n(\lambda)$ of a material defines how light (of a certain wavelength $\lambda$ ) propagates through this medium as compared to through vacuum. The propagation of light will change when it crosses the boundary between two media with different indices of refraction $n$; this ultimately results in effects such as refraction and Fresnel reflection - the basis of all lens-based optics. Whereas these effects can be used and harnessed to create an optical microscope, they can equally ruin an already composed image. For, once a perfect beam of light has been focused through the objective lens into an experimental sample, any distortions or aberrations of the beam occurring in between can only reduce the quality of the formed image. In an optical microscope these effects can easily be observed at the objective lenscoverslip-sample interfaces. In an optimal microscope configuration discontinuities in refractive index are avoided by matching the refractive indices of the immersion medium, coverslip and sample, thereby eliminating any Fresnel interfaces. Typically $\mathrm{n}=1.51$ is chosen, the refractive index of most standard optical glass. In general the average refractive index of biological samples deviates substantially from this value, resulting in non-optimal imaging conditions. In order to avoid Fresnel interfaces, the biological samples need to be fixated and embedded in special mounting media, thereby killing them in the process. This eliminates one of the main advantages of optical microscopy, namely the ability to non-invasively observe living organisms. Without this intervention, unfortunately, numerous image distortions come into effect, which severely limit the capabilities of observing the biological sample. In particular, the capability to image deep inside a biological sample becomes limited, due mainly to three effects that intensify the deeper inside the sample you try to image. These effects are spherical aberrations that are induced by a mismatch between the refractive index of the sample and the objective lens, light scattering caused by refractive index inhomogeneities and turbid samples, and bright fluorescence background signals caused by dense labeling or autofluorescence. ${ }^{72,76}$ The latter is independent of refractive indices and can be overcome with good labeling and sample preparation, as well as with confocal or two-photon microscopy

\footnotetext{
'Obviously, biological samples are in general a highly heterogeneous mixture of water, lipids, proteins and the like, each with an individual index of refraction. For simplicity, therefore, an average index of refraction is assumed, at which optical disturbances are minimal.
} 
(except for very deep measurements) ${ }^{77}$. Light scattering can be alleviated by using wavelengths in the far red or with the efficient detection of multiphoton setups (see also Section 3.4.2). In fairly benign samples, scattering effects become apparent only at depths greater than $50 \mu \mathrm{m}$. The impact of spherical aberrations can cause severe distortions even very close to the sample interface and need to be addressed directly. Therefore, if live organisms are to be examined without sacrificing image quality, either the refractive index of the optical components needs to be matched to the sample, the occurring aberrations compensated or both. For this the average refractive index of the sample needs to be determined.

\subsubsection{Estimating the average refractive index of the hippocampus}

Determining the refractive index of biological samples is by no means trivial. Biological samples are an inhomogeneous mixture of a multitude of different components with varying consistencies, each with a different refractive index. This necessitates an averaging over all the components as they are present within physiological conditions, without altering the proportions or the composition. Extreme care must therefore be taken, as unhealthy, damaged or dying cells will undergo profound changes from their healthy state. Also, desiccation, swelling, physical strain and protein denaturation will alter the sample consistencies. ${ }^{78}$ To further complicate matters, even tissue samples from a single organism, a mouse for example, can vary significantly in their optical properties when taken not only from different bodily regions, but also from different areas of a single organ, such as in the brain or merely within the hippocampal formation. Due to this difficulty, standard physical methods for determining the refractive index do not work. Combined with the fact that for most biological experiments it had not been necessary to push the limits of the optical resolution, very few actual measurements of the average refractive index of biological tissue have been performed. Indeed, for simplicity it is commonplace to assume a refractive index of $n=1.33$ for cells, following the reasoning that they consist mostly of water.

In order to roughly approximate the refractive of index of a biological sample, we shall simplify the system as far as possible. Biological tissue consists primarily 
Chapter 3. Imaging deep inside neuronal tissue by compensating spherical aberrations

of cells, which are basically lipid membrane hulls $(n=1.45)$ containing cytosol, a mixture of water $(n=1.33)$, proteins and DNA $(n \geq 1.50)$. Measuring the refractive index of cytosol is far easier than that of entire cells, and several studies place this value around $n=1.37,{ }^{79-81}$ accounting for the water, DNA and proteins in one. With this information we can further reduce the system to cytosol and lipid membranes, which places the refractive index of most physiological tissue samples somewhere between $1.37 \leq n \leq 1.45$. We can conclude that in tissue consisting primarily of large cells, the surface to volume ratio would place the refractive index closer to 1.37, whereas in fatty tissue, or in tissue containing many small cells or cell appendages, this value should be closer to 1.45 .

In 1989 Bolin et al. measured the refractive index of tissue samples from organs of several different species by replacing the cladding of a glass fiber with the homogenized tissue and measuring the angle of the exiting $(\lambda=633 \mathrm{~nm})$ light beam. ${ }^{78}$ Most tissue samples showed refractive indices in the range of 1.38-1.40, with notable exceptions at 1.36 (liver), 1.41 (muscle, kidney) and 1.45 (adipose tissue). They found almost identical values for intact tissue and homogenized tissue (i.e. tissue sent through a 'gentle' blender), as long as no coagulation (protein denaturation) took place; in this case the refractive index rose considerably. Also, they observed an increase in refractive index over the visible spectrum towards UV light by almost $\Delta n=0.3$.

More recently, other groups have measured the refractive index of biological tissue at different wavelengths using very different methods, such as computer tomography, phase microscopy or confocal microscopy. The resulting experimental values for the refractive index of mammalian tissue $(1.35 \leq n \leq 1.46)$ vary considerably when comparing tissue taken from different organs, but overall reproduce values between 1.37-1.40 for grey matter" of the brain.$^{82-84}$ In a very recent paper Sun et al. measured the refractive index of acute slices taken from intact rat brain tissue at $\lambda=1310 \mathrm{~nm}$ using optical coherence tomography (OCT). ${ }^{85}$

\footnotetext{
"The terms 'white' and 'grey' matter refer to the two distinct appearances of nervous tissue occurring in different regions of the brain or spinal cord. White matter consists mainly of myelinated axons, whereas grey matter consists mainly of the cell bodies and dendrites of neurons. White matter can be found primarily in the deeper regions of the brain and spinal cord, whereas grey matter is located further on the periphery of the brain, such as on the outsides of the cortex or hippocampal formation. ${ }^{5}$
} 
They found different values for white $(n=1.40)$ and for grey matter $(n=1.36-1.37)$. Also, they measured a sharp increase in refractive index for tissue that is under compressive strain, meaning that the liquids are pressed out of the cells, increasing the dry mass to water ratio and therefore the refractive index (by up to $70 \%-90 \%$ at very high strains). The measured values are slightly lower compared to those from previously mentioned experiments. But if the considerably longer wavelength, at which they were measured, is taken into account, they might actually be in good agreement. Concluding these deliberations we can make a rough estimate of the refractive index of hippocampal brain matter of $1.39 \geq \mathbf{n} \geq \mathbf{1 . 4 1}$ at wavelengths between $500 \mathrm{~nm}$ to $600 \mathrm{~nm}$.

\subsection{Measuring and compensating spherical aberrations}

As mentioned previously, there will be unavoidable discontinuities in refractive index at the multiple interfaces between the sample and the objective lens when imaging physiological brain slices in an optical setup. In the case of an inverse microscope there are the interfaces between the immersion medium, the coverslip and the sample; in an upright microscope the use of a coverslip can be bypassed by immersing the objective lens directly in the sample medium (in general an aqueous solution) and only one interface needs to be dealt with. Without a coverslip, however, this biological interface will typically not be flat, but curved to a varying degree. Aberrations in one of the media can be minimized by matching the refractive index of the immersion objective lens ${ }^{\text {III }}$ either to that of the coverslip or the aqueous solution, or one can try to match the refractive index of the sample itself. As our goal was to penetrate as deep as possible into nervous tissue, we chose the latter approach for our setup by using a glycerol immersion objective lens, which was optimized for use with samples of a refractive index of $n=1.45$. This value is slightly higher than the estimated value for our brain samples (Section 3.1), but should be a closer match than either a water- $(n=1.33)$ or an oil-immersion objective lens $(n=1.51)$. This will already strongly reduce the spherical aberrations generated inside

\footnotetext{
IIIThe refractive index of the objective lens itself does not change. Moreover, objective lenses are designed for use with a certain refractive index, which typically matches that of the recommended immersion medium.
} 
Chapter 3. Imaging deep inside neuronal tissue by compensating spherical aberrations

the sample. Moreover, the aberrations induced at the glycerol-coverslip interface are pre-corrected by the objective lens geometry. All that remains to be corrected, therefore, are the spherical aberrations induced by the remaining refractive index mismatch of the sample itself. Here, the objective lens' correction collar comes into play, which enables the correction of minor aberrations, such as can be induced by deviations in temperature, coverslip thickness or refractive index. ${ }^{86,87}$ If, therefore, we fix the temperature and coverslip thickness at appropriate values, then we can use the correction collar solely for compensating the remaining spherical aberrations induced by the sample.

\subsubsection{The correction collar}

The correction collar of the implemented glycerol immersion objective enables the compensation of minor spherical aberrations. The physical result of rotating the correction collar is the translocation of a group of lenses inside the objective, which induces spherical aberrations into the beam. In an ideal case these induced aberrations are equal but opposite to the aberrations encountered in the sample, thereby canceling each other out. The correction collar is specified for compensating deviations of the refractive index of the immersion medium of $\Delta n \approx \pm 0.01$, or to allow the use of coverslip thickness ranging between $140 \mu \mathrm{m}$ to $180 \mu \mathrm{m} .{ }^{87}$ Indentations on the correction collar arbitrarily divide the entire range into ten segments. We will refer to the setting of the correction collar as the correction value or $C V$, with steps ranging from one to ten.

\subsubsection{Distortions of the microscope point spread functions due to spherical aberrations}

In order to gauge the impact of spherical aberrations on our microscope and to determine the scope of our correction capabilities, we first evaluated the immediate effects on our excitation and STED beams. We visualized the point spread functions (PSFs) of our various beams using small, reflecting gold nanobeads. These samples were created by coating coverslips with a thin layer of Poly-L-Lysine to increase the adhesiveness of the surface, and then applying a small amount of gold colloid, resulting in a sparse layer of $80 \mathrm{~nm}$ gold beads on the surface. The optimal density 
was low enough to avoid bead clustering, but high enough to have several beads for comparison in a widefield image (roughly $10 \mu \mathrm{m} \times 10 \mu \mathrm{m}$ ). When illuminated by a laser beam, the reflecting beads reproduced the PSF of the incoming beam faithfully, allowing us to directly observe the system aberrations. We recorded the STED and excitation PSFs for different correction values in the optical (xy) plane, as well as along the optical ( $z$ ) axis in the $x z$ and $y z$ planes. The impact of the aberrations on the PSFs was clearly visible, as was the ability to minimize the aberrations (Figure 3.1).
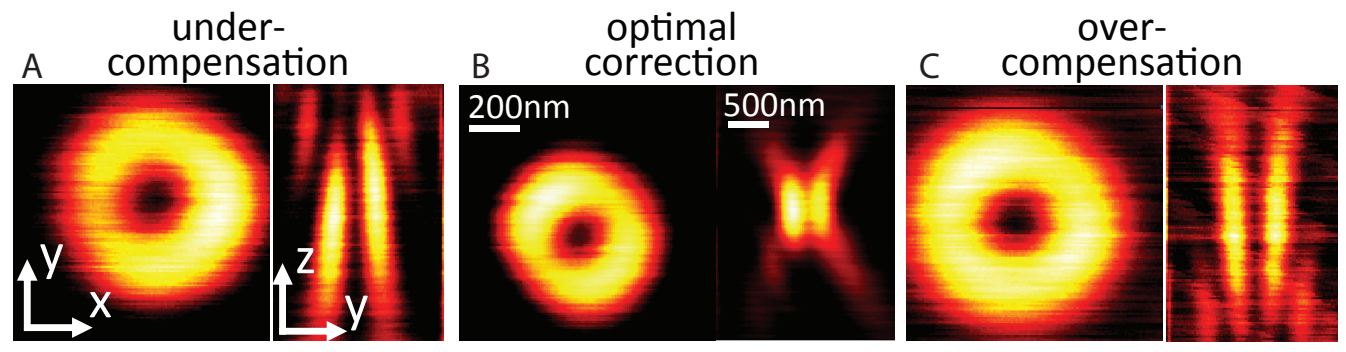

Figure 3.1. | Distorted point spread functions due to spherical aberrations. The effect of (A) negative and (C) positive spherical aberrations on a donut PSF are clearly visible when compared to (B) an optimally corrected system. The color table brightness was increased in (A) and (C) to better display the shape and extent of the aberrated PSFs.

Non-aberrated STED-donut PSFs (as produced using oil-immersion objectives) display a compact donut intensity profile in the optical plane with a clear intensity zero spot in the center. ${ }^{I V}$ Along the optical axis the donut lobes are parallel and evenly spaced, with a distinct zero intensity line running in between them; the lobes are compact in $z$, and, diagonally to the outside, the dimmer, symmetrical side lobes are visible. In the optimally corrected system with our glycerol objective, the PSF looked similar, but slightly larger in dimension, due to the lower numerical aperture of the glycerol objective lens as compared to typical oil immersion objectives (Figure $3.1 \mathrm{~b}$ ). ${ }^{\vee}$ If the induced spherical aberrations were not correctly compensated, then the PSFs are distorted visibly (Figure $3.1 \mathrm{a}, \mathrm{c}$ ). In the optical plane the STED donut increased in size and decreased in brightness. Much more striking were the distortions along the optical axis. Here, the donut lobes were stretched out along

\footnotetext{
${ }^{\text {IV }}$ Small donut-PSF sizes are desirable, as then the laser power is concentrated in a very small area. High laser powers in STED enable better resolution improvement.

${ }^{\vee}$ The $x y$ section of the donut here was not entirely symmetrical, which was due to a slight astigmatism inherent to the system. This had little to no visible effect on the STED resolution, however.
} 
Chapter 3. Imaging deep inside neuronal tissue by compensating spherical aberrations

the axis to multiple times their former size and were furthermore tilted with an angle towards each other. The opening angle depended on the magnitude and the sign of the spherical aberrations; negative aberrations caused an opening towards the bottom, positive aberrations towards the top. The laser power was now spread out along the entire PSF length, resulting in an overall decrease of peak intensity, especially in the focal plane. A considerable amount of light was transferred to the side lobes (either the top or the bottom side lobes, again depending on the sign of the aberrations), revealing the second or even higher orders of side maxima. Despite all these distortions of the PSF, however, the zero intensity minimum in the center of the donut remained largely intact. This was especially noteworthy, as the central donut zero is absolutely crucial for the resolution of any STED microscope. Importantly, this means that even with severe aberrations, the STED effect should still be visible using such a distorted PSF. Even though the STED resolution would be impaired due to the diminished power in the focal plane and the slight widening of the donut center, it would not be entirely eliminated. This phenomenon is a special property of the helical phase plate typically used to generate the donut PSF, which does not have any radial dependency of the phase pattern. Therefore, any radial distortions due to radially symmetric, spherical aberrations will not alter the relative phase along the entire optical axis and, in particular, not in the focal spot. In the excitation channel we observed much the same effects: the widening and dimming in xy, as well as the dimming and stretching in z. Due to the Gaussian intensity profile of the excitation spot, the tilting effect was not clearly visible, however. The observed aberrations of the PSFs were in good agreement with previous theoretical predictions. ${ }^{88,89}$ Furthermore, the apparently identical PSF distortions and optimal correction values for both blue and yellow wavelengths (excitation and de-excitation) agree well with previous calculations and experiments, showing that aberration effects are independent of wavelength, at least for minor penetration depths up to $\approx 40$ wavelengths. ${ }^{90,91}$ With the knowledge of the extent of these PSF distortions we were later able to recognize uncompensated aberrations in the system and then compensate for them. 


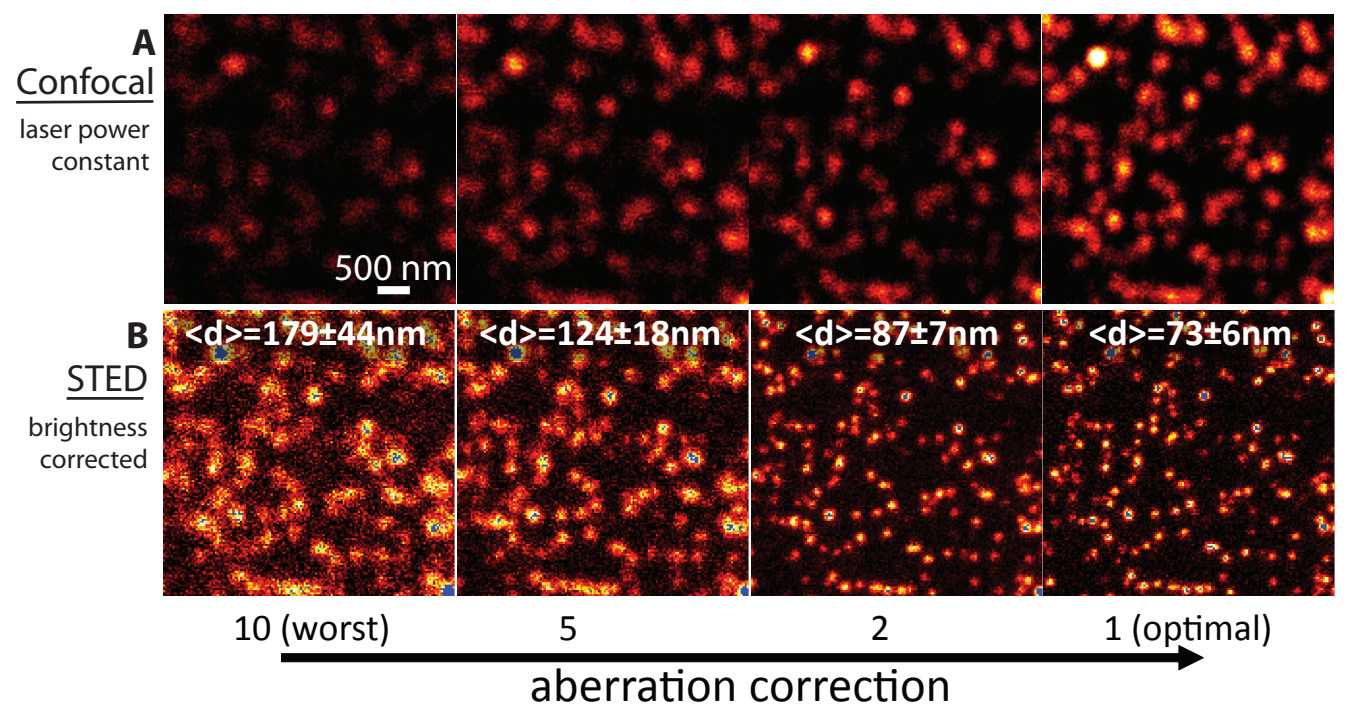

Figure 3.2. | Fluorescent beads imaged with varying degrees of aberration compensation. Uncompensated spherical aberrations are detrimental for signal brightness and spatial resolution for both confocal and STED imaging. (A) Uncorrected aberrations severely diminish the fluorescence signal, which can be seen by keeping the excitation intensity constant. (B) Even if the dimming is compensated with a stronger excitation, the spatial resolution decreases markedly as the spherical aberrations increase, especially for STED nanoscopy.

\subsubsection{Aberration compensation for confocal and STED imaging}

In the next step we studied the impact of spherical aberrations on actual confocal and STED images. For this we again prepared bead samples, but instead of gold colloid we applied a thin layer of fluorescent beads. These beads are small polymeric spheres filled with a fluorescent dye; for these experiments we used $40 \mathrm{~nm}$ large spheres filled with a yellow-green emitting dye. Because these beads tend to aggregate in the colloidal solution, they needed to be sonicated thoroughly before applying them to the coverslip. We imaged small $18 \mu \mathrm{m} \times 18 \mu \mathrm{m}$ areas covered in fluorescent beads, again using the entire range of the correction collar to compensate aberrations to a varying degree and to determine the optimal compensation. If optimally corrected we observed confocal resolutions of $190 \mathrm{~nm}$ to $200 \mathrm{~nm}$ and STED resolutions of $60 \mathrm{~nm}$ to $70 \mathrm{~nm}$ at de-excitation powers of $\approx 40 \mathrm{~mW}$ 
Chapter 3. Imaging deep inside neuronal tissue by compensating spherical aberrations

in the objective aperture. The STED resolution was slightly worse than can be expected from this laser power, because the wavelength of the de-excitation laser was optimized for use with EYFP and not for the yellow-green dye in the fluorescent beads. The yellow-green dye is blueshifted by roughly $10 \mathrm{~nm}$, resulting in noticeably less STED efficiency of the $595 \mathrm{~nm}$ de-excitation beam.

The aberrations clearly distorted the images in the expected ways (Section 3.2.2). When imaged confocally at constant laser power the recorded signal dropped significantly if the correction value was further than two steps away from the optimal position (Figure 3.2 a). This went hand in hand with a diminished signal-tonoise ratio. The full-width at half-maximum (FWHM) of the imaged beads increased slightly in the xy-plane, but was stretched significantly along the optical axis. With activated STED beam the effects were even more dramatic, due to the interplay of not one but two aberrated beams. Besides the observed dimming and lengthening of the FWHM along the axis, the subdiffraction resolution in the optical plane became severely compromised with increasing aberrations. Even when the reduced signal was compensated with increased excitation powers, the STED resolution decreased from optimal $60 \mathrm{~nm}$ to $70 \mathrm{~nm}$ to around $170 \mathrm{~nm}$ to $180 \mathrm{~nm}$, almost to diffraction limited levels (Figure 3.2 b). Of importance is that the correction values were identical when determined by either confocal or STED imaging. The correction values were also identical when determined independently from either the dimming and z-lengthening effects and matched the correction values determined from the PSF measurements (Figure 3.3). This meant that the system could be calibrated using purely confocal images, thereby reducing the illumination intensity and concomitantly alleviating bleaching and photodamage problems inside the more delicate live samples. Furthermore, the calibration could be done in live, fluorescent samples either by maximizing the image brightness (whilst considering possible bleaching artifacts) or by minimizing the observed length along the $\mathrm{z}$-axis, whichever proved to be more convenient.

The aberration compensation was more difficult when determined using structures in three-dimensional systems. One major problem was caused by system instability during operation of the correction collar. Because the correction ring is located on the objective lens itself, by necessity the objective will be moved slightly at 


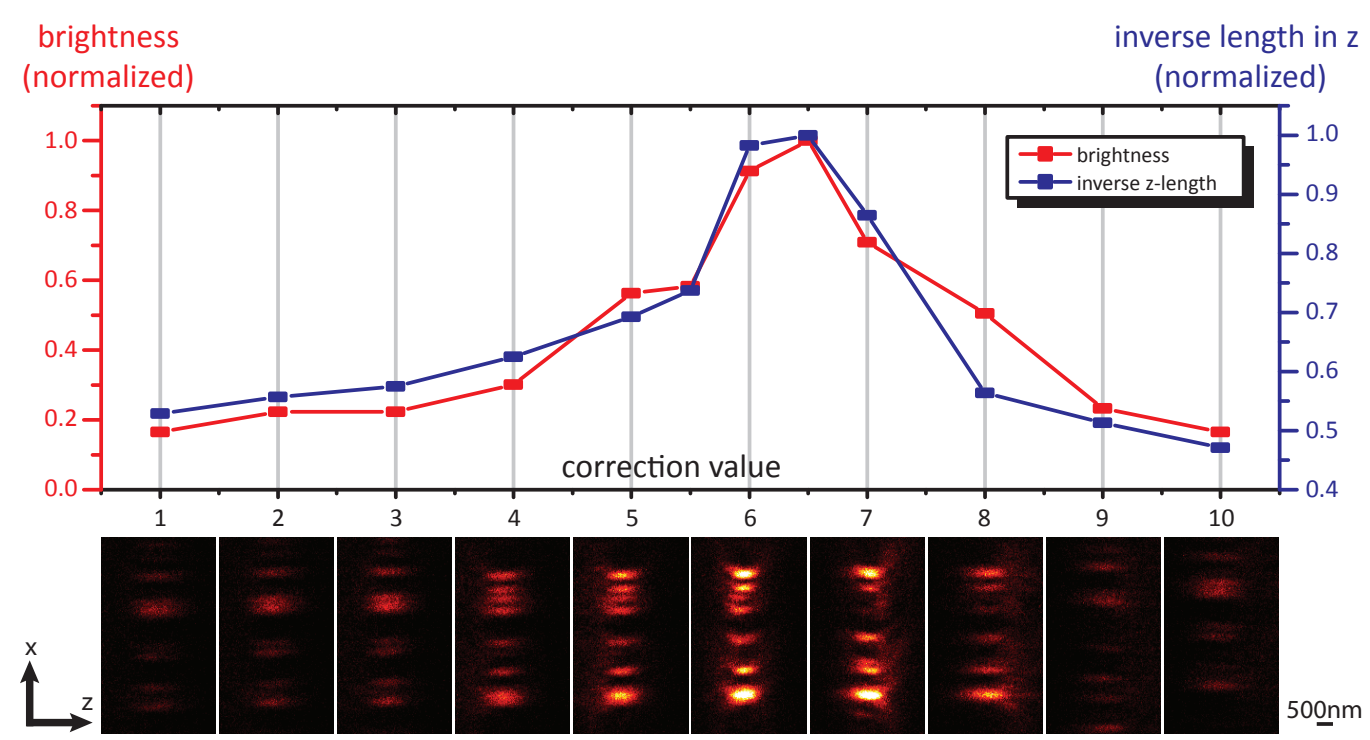

Figure 3.3. | Calibrating the correction collar by imaging fluorescent structures was possible either by maximizing the recorded signal of a structure, or by minimizing the apparent length in z. Here, fluorescent beads were imaged along the xz plane for different correction values. The peak intensity and the inverse length of the beads along the $z$-axis were normalized and plotted against the correction value (CV) of the objective. The maxima of both curves overlap, giving identical results of the optimal correction $\mathrm{CV}=6.5$.

each step of the manual alignment process. Even in a fairly stable system, minute changes of the objective position could move the imaged structure outside the field of view, requiring the image frame to be recentered. In densely labeled systems the exact feature used for calibration might be difficult or time-consuming to relocate, especially if structures that are indistinguishable in the widefield were used, such as fluorescent or even reflecting beads. Further complications arose from the aberration-induced length distortions along the optical axis, which caused structures to shift in and out of the focal plane as the aberrations changed. ${ }^{88}$ To compensate this effect, we recorded stacks of frames spanning several $\mu \mathrm{m}$ when adjusting the correction by maximizing the fluorescent signal of a structure best visible in the xy-plane. This way the imaged structure remained in the imaged volume, despite occurring drifts in $z$. Adjusting the correction by visualizing distortions in $z$ was also not trivial, due to the instability of the system during correction. If the imaged structure, such as a dendritic shaft, varied more strongly in width and brightness along its length than the slight changes induced by spherical 
Chapter 3. Imaging deep inside neuronal tissue by compensating spherical aberrations

Figure 3.4 | Dendritic spines imaged with and without aberration correction. (A) Aberration corrected confocal images already reveal considerable detail of dendritic spines. (B) STED images without pre-correction using the correction collar show slightly improved subdiffraction resolution, but suffer from low image contrast. (C) When corrected, STED images of dendritic spines reveal prior hidden details, such as the curvature of the spine head cup and inhomogeneities in neck width.

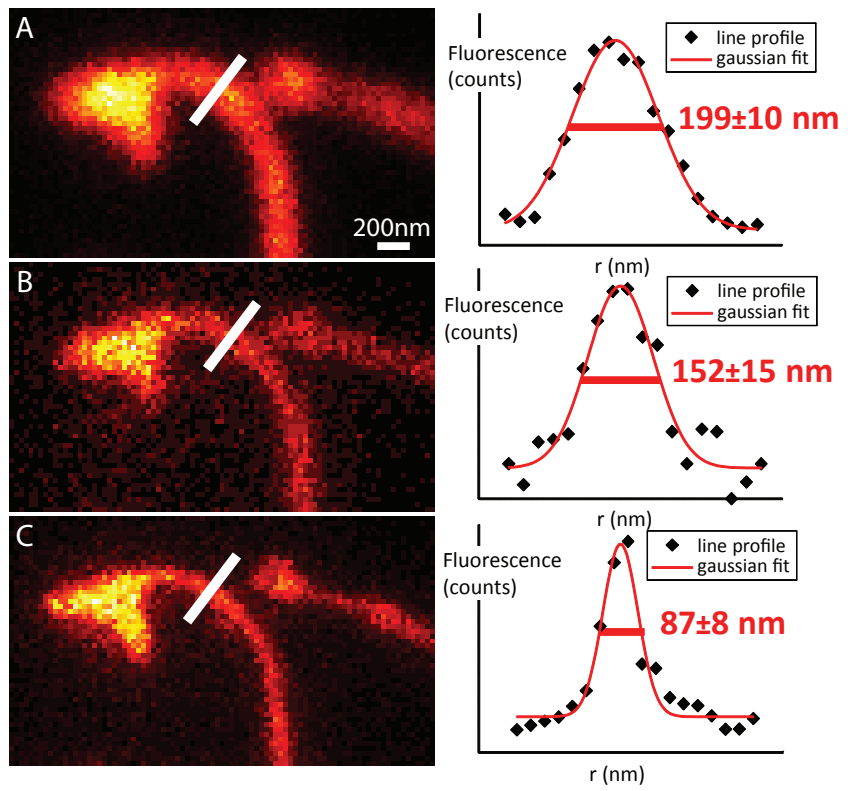

aberrations, then manual shifts could ruin a measurement. Nevertheless, the correction procedures tested on two-dimensional samples could be applied in live brain samples. The benefits of proper aberration correction were clearly evident in the recorded images, as can be seen in Figure 3.4.

It was not possible to establish any universal calibration curve of imaging depth over correction value. Even for artificial systems, such as three-dimensional samples consisting of beads embedded in Moviol (refractive index of $n=1.45$ ), the measured calibration curve varied notably from sample to sample. A rough correction correlation of 1 unit / $10 \mu \mathrm{m}$ could be determined, but this was never more than a rule of thumb. This became increasingly clear when imaging deep inside live brain slices, which were by nature far more heterogeneous than the artificial systems. Optimal correction values for individual locations at the same depth but at different lateral positions within a brain slice could vary considerably. The deviations were more prominent when comparing image locations from different anatomical areas inside the hippocampus, reflecting the high variability of the hippocampal structure. Regions with a higher density of soma demanded different correction values than regions with dense axon pathways or a higher density of dendritic arbors. Not all locations in a slice were suitable for imaging: local anomalies in the brain slices, such as pockets of water between the coverslip and brain slice, possibly coinciding 
with a strongly curved tissue interface could induce uncorrectable aberrations. Also, groups of dead or dying cells also hindered or even prevented imaging, as they rapidly became opaque and swelled, leading to severely increased scattering along with strong drifts of any structure in the immediate area. All in all, the strong inhomogeneity characteristic of the hippocampal brain slices necessitated separate aberration correction procedures for each individual image location. According to the nature of spherical aberrations, individual corrections could almost be ignored close to the coverslip but became increasingly obligatory at large imaging depths.

\subsubsection{Optimizing experimental parameters}

Understanding the optical consequences of the aberrations, we could explore the effects of easily variable experimental parameters, such as coverslip thickness, immersion media, heating and coverslip tilt, on the correction and then select the ideal settings for the appropriate situation. To do this we again used gold nanobeads, as they are by nature impervious to bleaching and can reveal more nuances of the occurring aberrations (especially non-spherical).

\subsubsection{Coverslip thickness and immersion medium}

Varying the coverslip thickness or the refractive index of the immersion medium had a severe impact on the spherical aberrations of the entire system. The glycerol objective lens was pre-corrected to compensate fixed aberrations incurred by specific values of both coverslip thickness and refractive index. So altering either parameter could be regarded as introducing (or removing) an additional aberrating layer, thereby shifting the dynamic correction range up or down by a fixed amount. ${ }^{92}$ Thinner coverslips would additionally increase the effective working distance of the objective lens, which constituted a hard limit for the maximal penetration depth inside a sample.

We measured these potential shifts to the dynamic correction range by observing the beam PSFs using gold nanobeads for different coverslip thicknesses $(70 \mu \mathrm{m}$, $140 \mu \mathrm{m}$ and $170 \mu \mathrm{m})$ as well as for different immersion media. We tested glycerol with varying water content $(1.44 \leq \mathrm{n} \leq 1.46)$, because added water reduces the refractive index, as well as 'refractive index liquids' with fixed refractive indices 
Chapter 3. Imaging deep inside neuronal tissue by compensating spherical aberrations

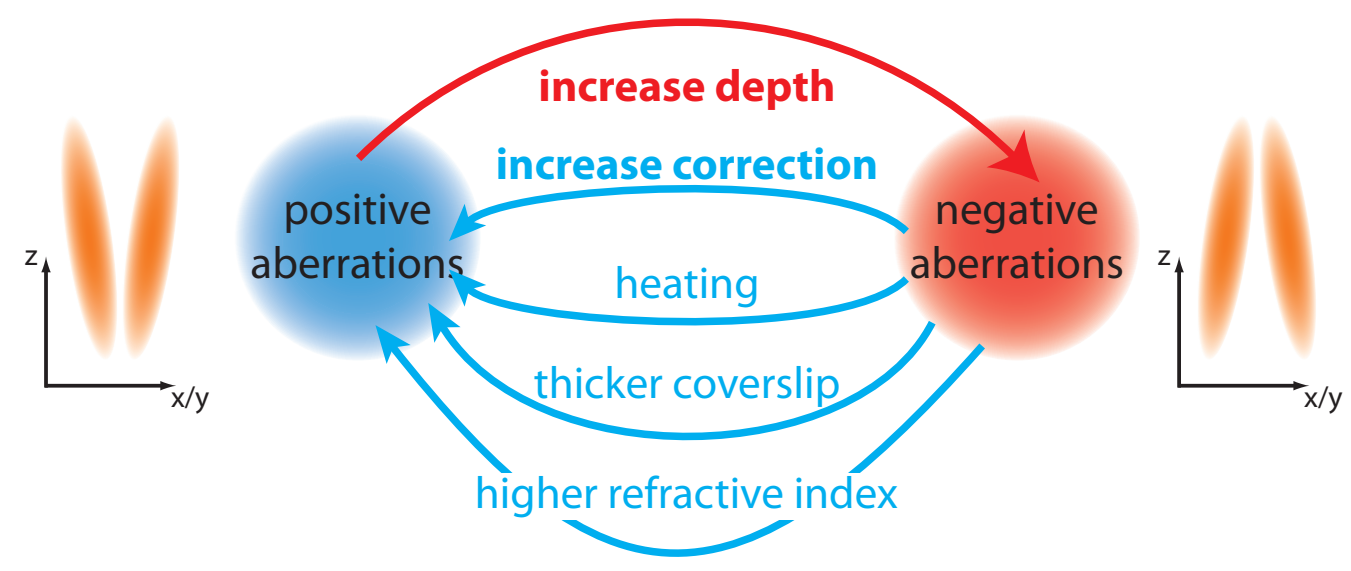

Figure 3.5. | Shifting the correction range by altering experimental parameters. Additional spherical aberrations can be introduced by altering some of the experimental parameters, such as the coverslip thickness and the refractive index of the immersion medium. This shifts the range in which the correction collar can compensate aberrations, potentially offsetting the increasing aberrations incurred by imaging deeper and deeper inside dense neuronal tissue.

$(1.43 \leq \mathrm{n} \leq 1.47)$. Obviously, if the additionally induced aberrations were greater than the correction capabilities of the objective lens, then we were not able to precisely quantify the ideal correction value. By varying two parameters with opposite effects simultaneously, however, it was possible to keep the optimum within the quantifiable correction range of the objective lens. Together, this allowed a quantification of the individual effects.

We observed increasing shifts toward lower optimal correction values for thicker coverslips as well as for immersion media with a higher refractive index than $n=1.45$. Changing the refractive index by $\Delta n=0.01$ shifted the correction value by more than a full rotation of the correction ring $(\Delta c v<-10)$. Changes in coverslip thickness induced lesser aberrations, with $\Delta d=15 \mu \mathrm{m}$ shifting the correction range by $\Delta c v \approx-1$. Heating the setup from room temperature $\left(21^{\circ} \mathrm{C}\right)$ to more physiological temperatures $\left(35^{\circ} \mathrm{C}\right)$ also caused the correction range to shift towards lower values by $\Delta c v \approx-2$ (Figure 3.5). Shifts to lower correction values imply the introduction of positive aberrations, necessitating additional negative aberrations to be introduced by the correction collar in order to compensate. 
Finally, we tested the viability of using two 'sandwiched' $70 \mu \mathrm{m}$ coverslips, with a thin layer of air, water or TDE (2,2'-thiodiethanol $\left.{ }^{93}\right)$ in between. This kind of configuration might be of interest for experimental situations in which the brain slices need to be replaced rapidly and/or frequently, as is commonly the case in patch-clamping experiments. Such experiments would therefore be slowed down considerably by the necessity of glueing each individual slice into the chamber (and to remove the previous slice and clean the chamber afterwards). Overall, the results were quite encouraging. The additional aberrating layer in between both coverslips introduced only small shifts in correction $(\Delta c v \approx+1$ for ACSF and $\Delta c v \approx-1$ for TDE). Furthermore, the length of the PSFs along the optical axis matched the results from single $(140 \mu \mathrm{m})$ coverslip samples, indicating a comparable quality of the correction. What was evident, however, was a reduced intensity and a worse signal to noise ratio. An explanation for this might be the Fresnel reflection occurring at the two additional refractive index discontinuities at the coverslip-medium interfaces.

\subsubsection{Coverslip tilt and unstable immersion media}

An additional cause of PSF distortions when using a non-oil immersion objective lens arises when the coverslip is tilted in relation to the optical plane. In the case of a glycerol immersion objective the tilt needs to be kept within $0.5^{\circ}$ in order to limit the intensity losses of the STED beam to less than $10 \% .{ }^{94}$ We could easily observe these distortions using gold beads when viewing $x z-$ and $y z-$ sections of the otherwise rotationally symmetrical PSF of the STED donut. Depending on the direction of the tilt, one of the donut lobes would be distorted and drawn out in a crescent-shape along the direction of the side lobes. We could avoid this to a surprising degree by meticulously eliminating any unevenness and tilt of the microscope stage and the sample chamber. Even though the coverslips with the brain slices were all glued in by hand, almost no tilt towards the optical plane was visible. This was more difficult when observing two 'sandwiched' coverslips, as discussed earlier. Here we observed slightly more pronounced tilts more often, yet still not severe enough to cause a noticeable loss of image quality.

We identified an additional source of aberrations when working with self-mixed glycerol as immersion medium. Over the course of several hours during an experiment we observed a gradual increase of negative spherical aberrations, as 
Chapter 3. Imaging deep inside neuronal tissue by compensating spherical aberrations

the optimal correction value shifted upwards. We traced this effect back to a gradual decrease of the refractive index, as the hygroscopic glycerol drew water from the air, thus self-diluting. This caused severe problems over the course of long experiments, requiring regular re-alignment procedures to be undertaken. We could easily prevent this problem by either using a refractive index liquid or by using commercial immersion glycerol, which had an added agent, preventing the glycerol from drawing water from the air.

\subsection{Measuring the spatial resolution deep within brain slices}

By using the correction methods described above we optimally compensated for spherical aberrations that were induced by imaging deep inside dense brain tissue. As demonstrated with the fluorescent beads (Section 3.2.3), the spatial resolution is a good measure for the effectiveness of the aberration correction. We estimated the spatial resolution of the microscope by measuring the diameters of thin dendritic spine necks. Spine neck widths vary from $40 \mathrm{~nm}$ to $500 \mathrm{~nm}$, as was measured with electron microscopy $;{ }^{95,96}$ therefore, only the thinnest spine necks were suitable for measuring the resolution of the aberration-corrected system. The spine necks of CA1 pyramidal neurons are among the thinnest in the hippocampus, and were therefore our primary imaged structures. Spine necks could be discerned easily because of high signal-to-noise ratios. This was caused both by low background levels due to very sparse labeling, as well as by the actin label, which caused the spine necks to be fairly bright compared to the much more voluminous parent dendrite. Dendritic spine necks are too thin to be observed properly using diffraction-limited optical techniques; therefore, electron microscopy has been the technique of choice, although it can only be performed in fixated samples. Scientifically, the plasticity of the spine necks and the (morphological) reaction to stimulation of the associated spine are hot topics of current research; these phenomena can only be observed in live cells. 

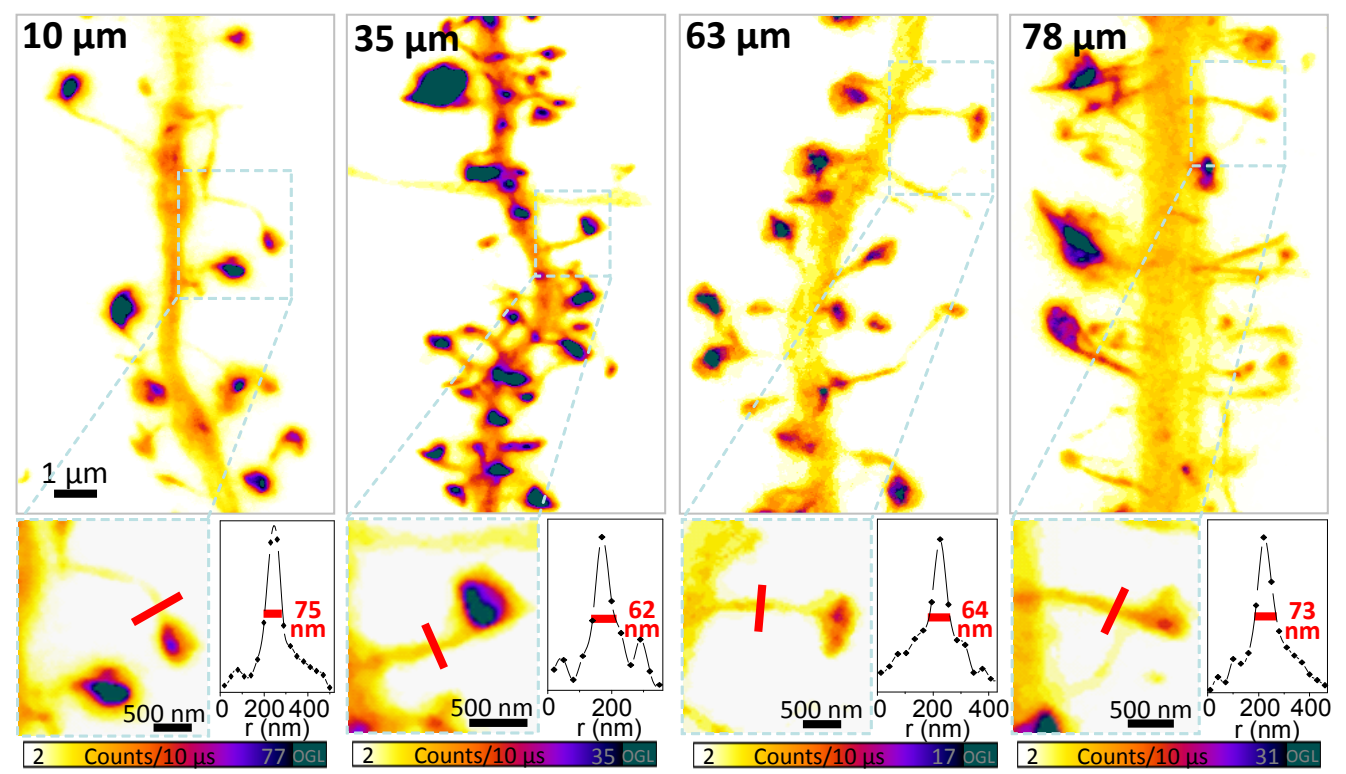

Figure 3.6. | Dendritic spines recorded at increasing depths beneath the tissue surface. By compensating the spherical aberrations that were induced by refractive index mismatch, dendritic spines could be imaged in high detail, even in great depths. Displayed are $20 \mu \mathrm{m}$ large stretches of spiny dendrites from various depths beneath the slice surface; the boxed areas show individual dendritic spines in higher magnification, whose neck width were measured using line profiles.

\subsubsection{Measuring the spine neck diameter}

To estimate the resolution achieved in our samples we imaged stretches of spiny dendrites at depths throughout the brain slices and then measured the widths of recorded spine necks (Figure 3.6). We determined the spine neck diameters by measuring the full-width at half-maximum (FWHM) of linear intensity profiles drawn across the spine necks. Several precautions were taken to prevent artifacts distorting the results. We used three-pixel-wide line profiles to guard against noise by negating the impact of single, unusually bright pixels. Furthermore, we measured the widths at up to four positions along each spine neck and discarded singular low results. To avoid misleading measurements of out-of-focus objects, we recorded stacks of optical sections that encompassed the entire dendritic section within the imaged volume. Optical sections were typically $500 \mathrm{~nm}$ apart and spanned $1.5 \mu \mathrm{m}$ to $4.5 \mu \mathrm{m}$, depending on how flat the imaged dendrite was. The optical sections were 
Chapter 3. Imaging deep inside neuronal tissue by compensating spherical aberrations

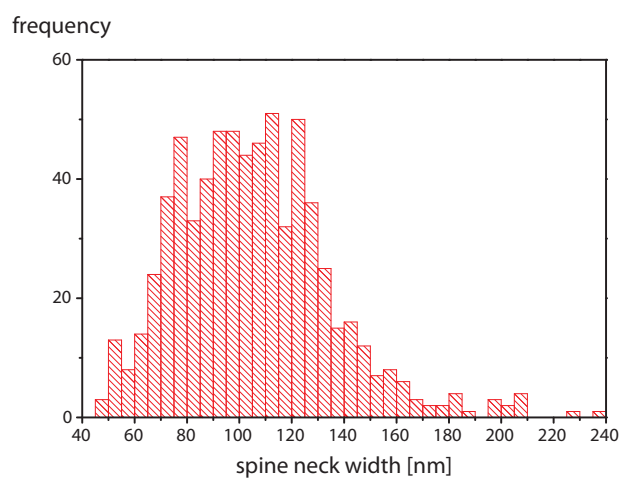

(a) complete measured range

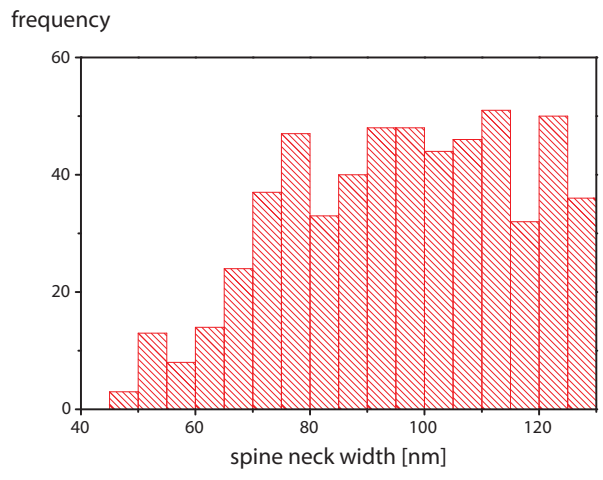

(b) thin spines only

Figure 3.7. | Measured distribution of spine neck widths The diameters of thin dendritic spines from CA1 pyramidal neurons were measured with STED microscopy. (a) Because we visually excluded thick spines, the distribution is artifactually skewed towards thinner neck values. (b) In the lower range we counted an even distribution of neck widths above $70 \mathrm{~nm}$, below which the frequency falls off quickly.

then reduced to two dimensional images by creating maximum intensity projections along the optical axis. All width measurements were performed on maximum intensity projections, and only if the dendritic spines were fully contained in the focal volume. Each of these steps was performed using the ImageJ software package.

In total we imaged spiny dendrites from over 40 neurons and measured the diameters of more than 500 dendritic spines. We concentrated on measuring thin spines, by visually rejecting any thick spines that were clearly close to or above the diffraction limit of $190 \mathrm{~nm}$, and measuring all the other (smaller) ones. Spine neck diameters generally varied locally along the whole stretch of the spine neck; the extent of these inhomogeneities ranged from small fluctuations to unmistakable bulges or constrictions (this will be discussed in Section 4.4.2). Because we needed structures for determining the spatial resolution, we did not measure at arbitrary locations when determining the width of a spine neck, but at the visibly thinner segments. Consequently, the observed spine neck distribution was skewed towards thinner diameters (Figure 3.7); the spine neck frequency was roughly constant between $70 \mathrm{~nm}$ to $130 \mathrm{~nm}$, after which it tailed off drastically due to the selection procedure and almost vanished beyond the confocal resolution of $190 \mathrm{~nm}$. On the 
opposite side, the frequency fell off steeply for very thin neck widths between $40 \mathrm{~nm}$ to $50 \mathrm{~nm}$. Less than $10 \%$ of the measured spine necks were thinner than $50 \mathrm{~nm}$ to $60 \mathrm{~nm}$ and therefore small enough to qualify as a measure for the spatial resolution. On average, 10-15 thin spines were observed on a given dendrite, meaning that in each image up to three spines were thin enough to be used.

Even though the hippocampal sections were originally cut $400 \mu \mathrm{m}$ thick during preparation, they thin out greatly during the first days and weeks in culture. ${ }^{52}$ In the experiments we discovered labeled neurons in depths up to $120 \mu \mathrm{m}$. The dendrites originating from these deep neurons did not extend flat in the optical plane, but were curved strongly and stretched out along the z-axis; this possibly indicates that they were located at the upper slice surface. There were far fewer transfected neurons deep inside the slice than close to the coverslip; the deeper one looked, the less there were. Therefore the frequency of very deep spines was significantly lower than of those further up.

\subsubsection{Resolution as a function of imaging depth beneath the surface}

In each depth range, we averaged the widths of the thinnest spine necks to estimate the spatial resolution of our microscope. For this we divided the data into $20 \mu \mathrm{m}$ wide bins; exceptions were the thinner superficial layer $(10 \mu \mathrm{m})$ and the deepest layer $(30 \mu \mathrm{m})$. In each layer the five lowest values were averaged for STED and for confocal data alike (Figure 3.8). Close to the coverslip we estimated confocal resolutions of $\approx 195 \mathrm{~nm}$ and STED resolutions of $\approx 60 \mathrm{~nm} .^{\mathrm{VI}}$ The resolution did not decline but remained constant even when penetrating deeper into the sample, up to depths of $95 \mu \mathrm{m}$. At this depth the aberrations became too strong to be compensated fully, and from here on both the confocal and the STED resolution began to decline gradually. Also, the image contrast worsened progressively, both in form of higher background fluorescence and a diminishing signal intensity, which could not fully be compensated by higher excitation powers. The deepest images, taken at depths of $120 \mu \mathrm{m}$, displayed a confocal resolution of $220 \mathrm{~nm}$ and STED resolution of $80 \mathrm{~nm}$.

\footnotetext{
${ }^{\mathrm{VI}}$ The errors for the confocal values were larger than for the STED data, because far fewer confocal images were taken and subsequently analyzed.
} 
Chapter 3. Imaging deep inside neuronal tissue by compensating spherical aberrations

A plot including additional spine neck measurements can be found in the appendix (Figure A.1).

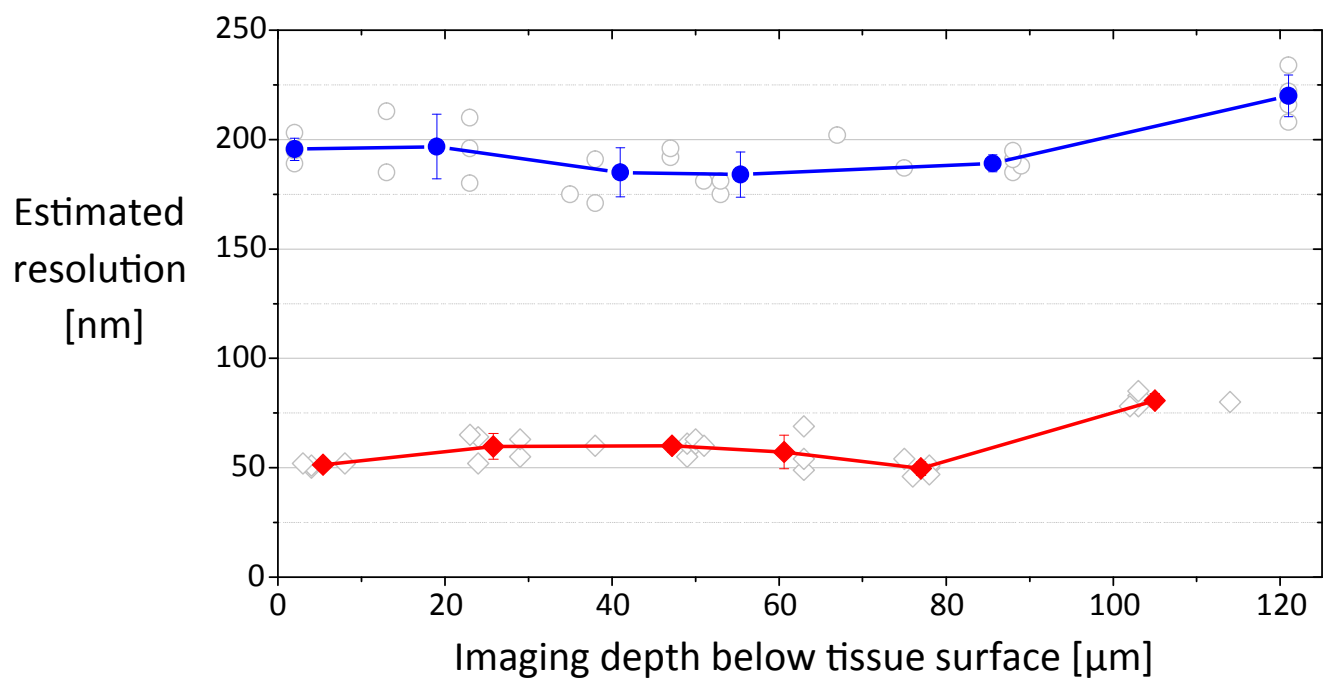

Figure 3.8. | The estimated spatial resolution deep inside dense brain tissue is shown for both confocal (blue) and STED imaging (red). Displayed are the five thinnest measured spine neck values (FWHMs) for both confocal (open circles) and STED (open diamonds). An average of these values is displayed as a guide to the eye for confocal (closed blue circles) and STED (closed red diamonds). Resolutions of at least $190 \mathrm{~nm}$ for confocal and $60 \mathrm{~nm}$ for STED could be sustained up until depths of $95 \mu \mathrm{m}$ after which the aberration correction capabilities reached their limits. The resolution declined gradually from here on; the maximum recorded depth was $120 \mu \mathrm{m}$.

When imaging within brain slices, the correction collar needed to be adjusted for each imaging location (Section 3.2.3). For this we selected small, bright structures, which we imaged using different correction values. Depending on the structure, we either imaged along the optical axis and measured the apparent length in z, or we recorded stacks in the optical plane and selected for maximum pixel brightness. As we proceeded to greater imaging depths, the spherical aberrations had to be compensated for by using ever higher correction values. Since higher correction values induce positive spherical aberrations, the aberrations in the sample must have been negative, in order for them to cancel each other out. At depths of $40 \mu \mathrm{m}$ to $50 \mu \mathrm{m}$ the aberrations were too strong to be corrected for solely with the correction collar; this was the maximum correction range when using $n=1.45$ 
glycerol as immersion medium. The correction range could be extended, however, by using an immersion medium with $\mathrm{n}=1.46$ (or higher) to induce additional positive aberrations (Section 3.2.4). Using a refractive index liquid with $n=1.46$ shifted the correction range deeper by about $50 \mu \mathrm{m}$. This enabled us to correct aberrations in the range of $10 \mu \mathrm{m}$ to $95 \mu \mathrm{m}$, after which we again reached the limit of the correction collar. Though it was possible to use immersion media with $\mathrm{n} \geq 1.47$ to correct at even greater depths, this severely complicated the pre-experiment alignment procedure of the microscope. The use of immersion media with $\mathrm{n} \geq 1.46$ shifted the correction range so deep, that aberrations close to the coverslip could no longer be fully compensated. This prevented nanobeads close to the coverslip surface being imaged without aberrations, as is necessary for proper alignment. Aligning using alternative methods was not possible in sufficient quality to offset the bonuses of using immersion media of $n=1.47$ or higher. The standard alignment was still possible for $n=1.46$ immersion media, because the residual aberrations close to the coverslip were small enough to be ignored. We therefore continued to use $n=1.46$ refractive index liquids at depths beyond $90 \mu \mathrm{m}$ to $95 \mu \mathrm{m}$, despite the inability to actively compensate the increasing aberrations. The deepest labeled neuron that we imaged was found $120 \mu \mathrm{m}$ beneath the sample surface.

\subsection{Discussion}

\subsubsection{Analysis and conclusions}

Can we be sure that the measured spine neck widths (Figure 3.7) accurately reflect the true distribution? If so, this can be true only for the lower end of the spine neck spectrum, because we selectively measured thin spines and excluded ones that looked too large. The apparent skew of the distribution towards thinner neck diameters and the tailing off towards thicker neck values reflected this. What about the lower boundary of the measured distribution, which we used to judge the spatial resolution? Whereas the neck widths were evenly distributed between $130 \mathrm{~nm}$ and $70 \mathrm{~nm}$, the frequency dropped off quickly from $70 \mathrm{~nm}$ to $50 \mathrm{~nm}$. This corresponds well to electron microscopy measurements on fixated samples that found no spine necks smaller than $40 \mathrm{~nm} .{ }^{95,96}$ If the spatial resolution of our microscope in tissue, however, had been insufficient to resolve these small structures, we would have 
Chapter 3. Imaging deep inside neuronal tissue by compensating spherical aberrations

expected to observe peaking values at the actual resolution, followed by a sharp drop-off at thinner values; all structures smaller than the resolution would have appeared to be larger - of similar size than the true resolution. This peak would be far less obvious, however, if coinciding with a (sharp) decline instead of a flat distribution. The spatial resolution of our microscope was measured in Section 3.2.3 to be better than $60 \mathrm{~nm}$ to $70 \mathrm{~nm}$ for fluorescent beads on a coverslip. A detailed comparison of the spine neck widths measured using STED or electron microscopy would be interesting, as a method to evaluate the impact of fixation artifacts on the neuronal morphology.

The measured neck widths cannot be exact, however, considering that the spine neck widths were not constant but could vary over the entire length of the spine. Because we measured the thinnest section of each spine, the distribution can be considered to reflect a lower boundary of spine neck widths. These local fluctuations may have benefited the resolution measurement by increasing the chance to find a structure thin enough to be used as a measure. Furthermore, because our fluorescent label attached to actin, it was not possible to distinguish between the true morphology of the spine neck and the actin distribution. But because dendritic spines are highly enriched in actin, ${ }^{97}$ in thin spine necks this label should be similar in appearance to a volume label. VII To measure the lower boundary of the spine neck diameters more precisely, a microscope with a resolution better than $40 \mathrm{~nm}$ would be needed, as well as an unambiguous labeling, such as a membrane-bound fluorophore.

Given that the correction collar optimally corrects the image by inducing aberrations that are equal but opposite to the aberrations in the sample, we can deduce some information about these aberrations and their nature. In Section 3.2.2 we determined that higher correction values induce increasing positive aberrations, and in Section 3.3.2 that we need ever higher correction values to offset the aberrations in increasing depths beneath the sample surface. Consequently, the spherical aberrations induced by the refractive index mismatch sample were negative. This implies that the average refractive index of the brain tissue was lower than that of the glycerol immersion $(n=1.451){ }^{88}$ These observations are in good agreement both with our direct measurements of the refractive index of hippocampal tissue, as well

\footnotetext{
VII Regardless, this had no ramifications for the estimation of the spatial resolution of our STED nanoscope.
} 
as comparative tests of correction values with other refractive index liquids, thus further supporting our approximation of the average refractive index of hippocampal tissue.

We observed a clear loss of image contrast as the imaging depth increased beyond $80 \mu \mathrm{m}$; this was both due to increased background fluorescence and declining signal intensities. The reduced signal could not be fully offset by increasing excitation intensities. Whereas uncompensated aberrations could account for the reduced signal, they cannot explain the increasing, diffuse background that occurred even in very sparsely labeled areas. The most probable explanation is increased scattering, which should intensify deeper inside the dense brain tissue. The average scattering length inside grey brain matter is $95 \mu \mathrm{m},{ }^{17,98}$ thus close to this observed depth. The loss in image contrast was still tolerable at our maximum depths of $120 \mu \mathrm{m}$, but would surely constitute a serious problem at even greater depths.

The maximum depth to which neurons could be imaged in this setup was not inherently limited by insufficient aberration compensation. More basic restrictions were the cause, such as the maximum thickness of the brain slices and the limited working distance of the objective lens. The working distance of the glycerol immersion objective lens was $280 \mu \mathrm{m}$, but this could have been extended to a certain degree by using thinner coverslips and compensating the additional aberrations with a higher refractive index immersion medium. This, however, would have incurred further complications, such as severe alignment difficulty (as discussed in Section 3.3.2). The maximal thickness of the organotypic brain slices was limited by the technique: if brain slices were cut thicker than $400 \mu \mathrm{m}$, then the bottommost cells near the coverslip would not receive adequate nourishment from the medium and consequently die off. This could have been alleviated by using alternative protocols, such as cultivating the brain slices on porous membranes instead of glass coverslips, ${ }^{52,99}$ thus allowing nutrients to be taken in from both sides of the brain slice. In general, such cultured slices are thicker than slices used in our method and could possibly be grown even thicker. But these methods would have caused additional imaging problems, such as imaging through the membrane or necessitating an upright microscope. Other options include using acute brain slices or conducting in vivo experiments in live animal brains. Increased scattering can be 
Chapter 3. Imaging deep inside neuronal tissue by compensating spherical aberrations

expected in these cases. In acute slices there is a thick layer of dead and damaged cells at the interface, forming a dense, turbid layer with increased scattering and autofluorescence. In the living brain, scattering, motion artifacts and different tissue layers would need to be considered. But even with greatly enhanced depth penetration, this approach would still not be able to examine the hippocampus in vivo, which is locked deep inside the mammalian brain.

\subsubsection{Outlook and improvements}

This thesis describes a simple method for adapting nanoscopy methods for use in physiological neuroscience experiments. The demonstrated depth penetration of $120 \mu \mathrm{m}$ is sufficient for conducting experiments in near-physiological environments, such as organotypic and acute slices, as well as in in vivo experiments in the outer fringes of the brain, such as the cerebral cortex. Yet these depths are shallow when compared to the limits set by two-photon microscopy of $1 \mathrm{~mm}$ and more. ${ }^{17,100}$ For this gap to be lessened, further modifications are necessary that allow even deeper tissue penetration by tackling the problems of aberrations, scattering and background fluorescence.

Additional steps can be taken to further minimize sample-induced aberrations. The use of immersion media with refractive indices of $n \geq 1.47$ and thinner coverslips would be a quick, albeit highly problematic, solution. Among other problems it would require more sophisticated alignment techniques, and would limit imaging to the very deep areas. If we assume that the average refractive index of hippocampal tissue lies in the range of $1.39 \leq n \leq 1.41$, as both our measurements and the literature suggest (Section 3.1), then other objective lenses could provide a lesser refractive index mismatch. A recently developed objective from Olympus uses silicone oil as immersion medium ( $\mathrm{n}=1.41, \mathrm{NA}=1.3,60 \mathrm{x}$, UPLSAPO 60XS, Olympus), which should prove a closer match to brain tissue. This objective also features a correction collar, so it could be handled similarly to the glycerol objective. The closer refractive index match to brain should induce fewer spherical aberrations when penetrating deep into tissue, but would increase the detrimental effects of coverslip tilt, due to the higher mismatch to optical glass. 
An even higher level of aberration correction might be achieved by a completely new approach using adaptive optics technology. Adaptive optics approaches, originally practiced in astronomy, involve measuring the deformation of the wavefront and then correcting these defects using a deformable mirror or a spatial light modulator (SLM). ${ }^{89}$ These wavefront corrections not only compensate spherical aberrations, but also all other modes of aberrations. These adaptive optics techniques are readily compatible with nanoscopy techniques ${ }^{101,102}$ and, given the right algorithms, they could be conducted automatically and quickly. As a further bonus, the correction collar would not need to be operated during delicate measurements, thus greatly improving the system stability.

The two other problems that need to be considered when attempting to go ever deeper into tissue, are optical scattering and background fluorescence. A typical approach to reduce scattering is to use wavelengths in the deep red and infrared spectral range. Optical scattering decreases with increasing wavelength, but in practice far less than the commonly quoted $\lambda^{-4}$ dependency of Rayleigh scattering would predict. ${ }^{17}$ Increasing the wavelength of our setup from $488 \mathrm{~nm}$ to over $1000 \mathrm{~nm}$ has been found to reduce the scattering by half, ${ }^{98}$ not by a factor of 16 . The absorption, in any case fairly low, would not be decreased. A very powerful technique to reduce scattering in multiple ways is the multiphoton approach. In a multiphoton microscope fluorescence excitation occurs only in the focal plane, which means that any detected fluorescence photons must have originated from this location. Therefore, multiphoton microscopes can gather and use every photon incident into the objective lens, even if the photon has been scattered multiple times. In contrast, confocal microscopy rejects all scattered and out-of-focus photons, and only uses unscattered photons straight from the focal plane. Other benefits of multiphoton excitation include both less out-of-focus excitation, as well as less autofluorescent molecules that can be excited in this far-red spectral range. The required excitation powers are much higher, though, in the range of tens of $\mathrm{mW} ;{ }^{14,75}$ this leads to unwanted excitation at the sample surface, again limiting the penetration depth. ${ }^{77}$ Recently, two-photon microscopes have successfully been enhanced with a single-photon STED beam. Even though this greatly improved the resolution over previous two-photon methods, especially in flat samples, ${ }^{75}$ the resolution displayed in tissue depths of $40 \mu \mathrm{m}$ was still only $\approx 240 \mathrm{~nm},{ }^{74}$ 
Chapter 3. Imaging deep inside neuronal tissue by compensating spherical aberrations

and therefore still in the range of diffraction-limited confocal microscopy. Here, scattering was only reduced for the excitation beam, but not for the more delicate de-excitation beam. Yet the resolution of a STED microscope rises and falls with an

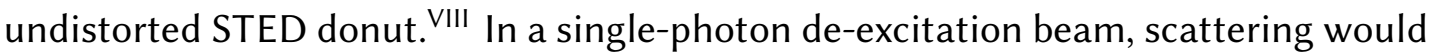
both decrease the overall intensity, as well as scatter photons into the donut center; both effects would seriously compromise the STED effectiveness. The obvious answer would be a multiphoton de-excitation beam. This is by no means trivial, and entails a number of problems that would need to be addressed. RESOLFT microscopy might be better suited for multiphoton de-excitation in this case, in part because of the otherwise immense de-excitation intensities required.

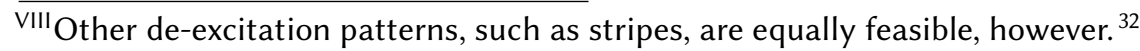




\section{Physiological nanoscopy of dendritic spines and actin substructures}

At birth, the principal morphology of the brain is fully established and the proliferation of neurons ceases. Yet, the development of the brain is far from completed, as the complex network of billions of interconnected neurons becomes ever more sophisticated. ${ }^{5}$ The neural circuitry can be rewired by altering the interconnecting neuronal junctions, the synapses. These alterations involve the generation of new synapses, elimination of old and the modification of existing ones. It is commonly believed that strengthening and weakening of synapses forms the basis of memory and learning in the brain. ${ }^{103,104}$ Most excitatory synapses in the brain are formed on small dendritic protrusions called spines, ${ }^{105}$ which are not static structures but undergo a tremendous amount of morphological change. ${ }^{106,107}$ Many psychiatric and neurological disorders, such as addiction, mental retardation, schizophrenia or Alzheimer's disease, are accompanied by changes in spine number and morphology. ${ }^{108,109}$ But also in healthy brains are dendritic spines highly plastic, even in adulthood. ${ }^{110,111}$ They can transiently change their volume by over $30 \%$ in a matter of seconds, ${ }^{112-114}$ and can durably alter their shape slowly over minutes to hours. ${ }^{115}$ The major driving force of rapid morphological change in spines is the structural protein actin. ${ }^{116-118}$ Actin is highly enriched and motile in dendritic spines, ${ }^{119}$ where it plays a number of important roles in synaptic activity, morphology and long-term changes. ${ }^{97}$ The modulation of actin dynamics is the driving force behind the morphological changes that are associated with changes in synapse strength and functionality. ${ }^{120-123}$ The observation of these morphological changes is very difficult, however, due both to the nanoscopic size of synaptic actin structures and because synapses are embedded deep within dense neuronal tissue. Synapses that are still integrated in a functional neuronal network cannot be removed without disturbing the delicate physiological environment. To image these sensitive structures, one therefore needs nanoscopic resolution coupled with good depth penetration - such as was demonstrated in the previous chapter. With our enhanced STED and RESOLFT nanoscopes we were able to observe nanoscopic substructures and rapid morphological fluctuations of dendritic spines and the actin cytoskeleton. Furthermore, we examined the effects of temperature and laser irradiation on the spine dynamics, and recorded the nanoscopic morphological responses of dendritic spines to drugs and chemical stimuli. 


\subsection{Lifeact label}

The labeling method we used for these experiments resulted in sparse labeling with low background fluorescence. The method involved injecting a modified Semliki Forest virus carrying an EYFP-Lifeact gene sequence into the intracellular space in hippocampal brain slices. Neurons in the injection site took up the virus and began expressing the EYFP-Lifeact label after 6-8 hours. Typically, less than a dozen neurons were transfected at each injection site. In the CA1 and CA3 regions of the hippocampus, the virus transfected mainly pyramidal neurons. Injection sites in the subiculum, a region close to the CA1, resulted in a mixture of transfected pyramidal and other neurons. If injected into the dentate gyrus, the virus transfected mainly granule cells. We distinguished between different neuron types using visual criteria, such as examining the morphology of the cell body and the protruding dendrites, the location of cell body within the hippocampal structure, and the orientation and extent of the dendritic arbor and, if visible, the axon. CA1 pyramidal neurons were identified by their small, mostly triangular-shaped soma and the typical dendritic arborization. CA3 pyramidal neurons could be discerned by their large cell bodies and the bright spiny excrescences on the apical dendrite close to the soma. Granule cells displayed smaller, round cell bodies, dendritic arbors that projected in one direction only (into the molecular layer of the dentate gyrus) and axons that projected into the $\mathrm{CA} 3$ region. By labeling multiple regions of the hippocampus, it was easier to unambiguously identify the desired brain region (typically the CA1).

The labeled neurons displayed very different brightness levels. Some types of neurons expressed the label more strongly than others, but there were also differences within one neuronal species. CA3 pyramidal neurons were much brighter than CA1 pyramidal neurons and dentate gyrus granule cells. We avoided imaging very dark CA1 pyramidal neurons, because they tended to be unhealthy and started blebbing after the first one or two confocal images." For imaging, we selected

\footnotetext{
'In most brain slices the hippocampal anatomy was clearly visible. In hippocampal sections from the peripheral regions, however, the anatomy was more complicated.

"Blebbing in neuronal processes occurs, when the cell membrane loses cohesion and starts to deform. In dendrites or axons, adjacent stretches either constrict or swell, resulting in the appearance of a chain of pearls.
} 
neurons with dendritic spines that were of comparable brightness to the dendritic shaft.

Transfected brain slices exhibited very low autofluorescence, all of which was visible in the yellow spectral region. In confocal images the autofluorescence was almost not detectable and disappeared completely in STED images. The autofluorescence was bleached entirely after exposure to the STED beam, as it could be excited by the wavelength of the STED light.

\subsubsection{Lifeact and cytosolic labeling compared}

We evaluated this labeling technique by comparing the Lifeact label to the cytosolic neuron label of Thy1-EYFP transgenic mice. This transgenic mouse strain expresses EYFP in the cytoplasm of projection neurons that express the Thy 1 gene, ${ }^{124}$ such as CA1 pyramidal neurons. The Thy1-EYFP brain slices displayed a very high density of labeled CA1 pyramidal neurons, typically with hundreds of labeled neurons in the CA1 region."I Neurons in both preparations were of similar appearance and displayed comparable amounts and shapes of dendritic spines. The architecture and organization of the cells in the CA1 region was also indistinguishable. These similarities suggest that neither the transfection method nor the Lifeact-EYFP-label had a significant impact on the morphology or viability of transfected neurons within the experimental imaging period of $\approx 36$ hours. This corresponds with observations of stable transgenic mouse strains expressing a fluorescent Lifeact label. ${ }^{125}$

Neurons that expressed the cytosolic label displayed clear traits of volume-labeled structures (Figure 4.1). Thick, voluminous structures were bright, whereas smaller features appeared much darker. The dendritic shafts were very bright, potentially eclipsing dimmer structures in the immediate proximity and a source of elevated fluorescence background. Dendritic spines were much dimmer. Thin spine necks were exceedingly dim, whereas spine heads, especially large ones, were brighter. The difference in brightness between the dendritic shaft and the spines could be reduced by imaging secondary or tertiary dendrites located far away from the soma

\footnotetext{
IIIOccasionally, granule cells in the dentate gyrus were labeled as well.
} 


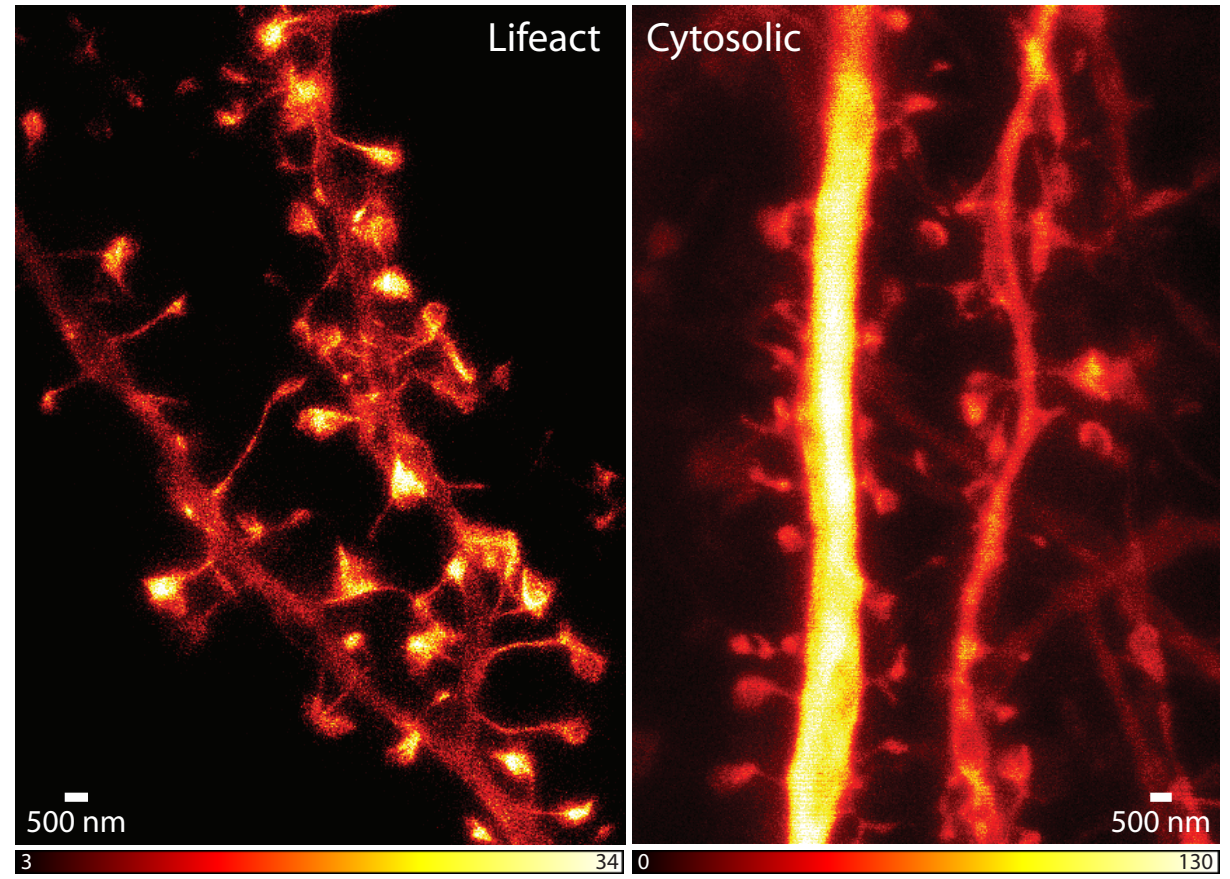

Figure 4.1. | Neurons labeled with EYFP-Lifeact compared to cytosolic EYFP are quite different in appearance. Neurons expressing the Lifeact-label exhibit very bright dendritic spines, which are highly enriched in actin, and comparatively dim dendritic shafts. Filaments and hotspots not directly related to the dendritic morphology can be observed. Neurons expressing a cytosolic label, however, display brighter signals in the most voluminous structures, such as dendritic shafts and large spines. Smaller structures, such as slender spines or thinner dendritic shafts, are much dimmer in comparison. Also, the density of labeled cells in the EYFP-Lifeact transfected cells is much lower, thereby offering better contrast. Both images were recorded with STED (raw data).

and therefore comparatively thin. Such slender dendrites offered good contrast for imaging the morphologies of dendritic spines.

The Lifeact-labeled neurons were starkly different in appearance. Most strikingly, dendritic spines were typically brighter than the associated dendritic shaft. Spine heads were considerably brighter, but even thin necks could be discerned clearly. The background fluorescence levels were low close to the dendritic shaft, offering good contrast for imaging dendritic spines. The morphology of the entire dendrite and the spines was visible, as for cytosolic-labeled neurons. The morphological outline was neither as bright nor as homogeneous, however. Contained in the volume of the dendrite and the spines were distinct, irregular inhomogeneities, such 
as small local hotspots, dark 'empty areas', thin filaments or even large bundles (see also Section 4.4). Spine heads and necks exhibited strongly disparate brightness levels, mostly irrespective of size. In dendritic shafts, long, thin cables were often visible running along the periphery. Moreover, large empty areas of different sizes appeared as dark ovals or long strips, ranging from several $\mu \mathrm{m}$ to more than $20 \mu \mathrm{m}$.

One great advantage of the Lifeact-EYFP label became apparent during long-term imaging experiments. Repeated imaging of individual locations could be performed without a significant loss in brightness; instead of dimming the fluorescence intensity remained constant. In part, this was due to the robustness of the EYFP label towards bleaching. The main reason, however, was the ability of the Lifeact-label to replenish. If we imaged one location for extended periods without interruption, bleaching of the structures was apparent. But given some time to recuperate and replenish, the brightness recovered to previous levels. This enabled the continuous observation of actin structures without signal loss and unaffected by bleaching artifacts.

\subsection{Imaging paradigms}

The living neuronal structures we observed in the brain slices were never entirely static, but shifted, moved and changed to varying degrees. IV When observing longterm structural changes, therefore, we took care to avoid drift-induced artifacts. We kept the observed structures inside the imaged volume by recording stacks of optical sections around the structure in question and refocusing between stacks, if necessary. This way, real movement and shape changes could be discerned from misleading effects caused by a drift in and out of the focal plane. In healthy slices, these drifts tended to be fairly slow and isotropic, noticeable only over the course of hours. Rapid movement of entire dendritic stretches usually occurred only after a stimulus, or in the case of extensive damage to the imaged neuron or unhealthy cells in the immediate proximity. Each frame was recorded in less than $20 \mathrm{~s}$, so entire stacks were imaged in 1-2 minutes. Hence, the imaging times were fast enough

\footnotetext{
${ }^{\mathrm{IV}}$ In this thesis we shall discriminate between the two concepts of structures exhibiting pure movement without simultaneously altering their morphology, and structures undergoing morphological changes while otherwise remaining in one position. Motility shall be used as a collective term for movement, changes or a combination of both.
} 
to ignore slow drifts. In case of fast movements, the individual stacks or frames were realigned using the stackreg algorithm of the ImageJ software package (see also Section 3.3).

Intense laser illumination, such as two-photon excitation, can cause damage to nervous tissue. ${ }^{126}$ Therefore, we took several precautions to limit potential cell damage when using STED to observe neurons over long time periods. We limited the de-excitation power to $33.6 \mathrm{~mW}$, which still allowed STED resolutions of $\leq 60 \mathrm{~nm}$. Moreover, we allowed the neurons some time to recover after each imaging stack. This gave the Lifeact label time to replenish and any possible disturbance of the neurons (e.g. heating, phototoxicity) to dissipate. These imaging breaks were typically in the range of 5-10 minutes when observing long-term dynamics. In the case of fast dynamics these imaging breaks were either very short (seconds to minutes) or omitted entirely. Antioxidants in the ACSF were introduced to intercept any free radicals created during imaging, which otherwise might induce phototoxicity. During the long-term experiments we did not observe any direct correlation between experiment duration and lethal cell damage.

\subsection{Latrunculin B effects on Lifeact-labeled neurons}

To verify whether actin was truly labeled in our slices, we exposed transfected neurons to the drug Latrunculin B (LatB), a toxin derived from the Red Sea sponge latrunculia magnifica. ${ }^{127}$ Latrunculin $^{\vee}$ passively causes depolymerization of actin filaments by binding to actin monomers in a way that greatly hinders them from polymerizing. ${ }^{68,128,129}$ Because cellular actin constantly assembles and disassembles, ${ }^{119}$ blocking the polymerization will passively lead to a depolymerization of filamentous actin. If our label is truly bound to actin, then dissolution of actin filaments should disperse the fluorescence signal evenly in the cytosol, eliminating any fluorescence hotspots. Furthermore, the removal of actin filaments, which are a substantial part of the cytoskeleton, will with great likelihood affect the neuronal morphology to some degree, especially in actin-enriched dendritic spines. ${ }^{130}$

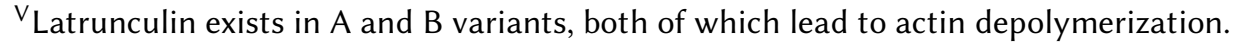




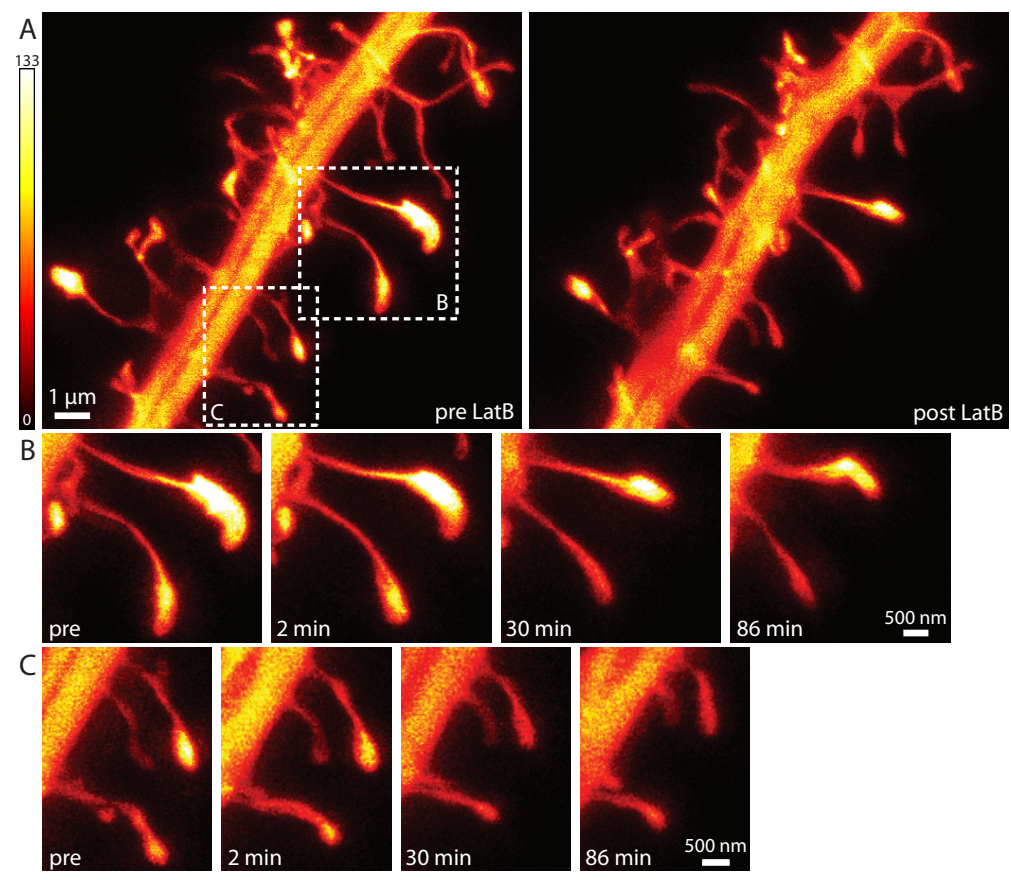

Figure 4.2. | Effects of Latrunculin B on dendritic spines from EYFP-Lifeact transfected neurons. Bath application of Latrunculin B (LatB) influences the morphology of dendritic spines and the concentration of filamentous actin. (A) STED images of a spiny dendrite belonging to a CA1 pyramidal neuron before (left panel) and after (right panel) washing in LatB. (B,C) Zoom-in of the boxed areas in (A) depicting dendritic spines undergoing change in a time-lapse series. The first image was recorded before neurons were subjected to LatB, the following images at subsequent times afterwards

Brain slices were exposed to Latrunculin by suffusing them with a modified ACSF buffer containing $10 \mu \mathrm{M}$ LatB for at least 10-30 minutes. The Latrunculin was then either washed out or maintained throughout the experiment. Individual dendrites were imaged repeatedly both before and after exposure to the toxin. Roughly ten minutes after the influx of Latrunculin, first changes began to occur (Figure 4.2). Fluorescence hotspots gradually grew dimmer and dissipated, whereas the brightness of the homogeneous labeling of the dendritic shafts remained constant or even increased. The brightening of the shafts occurred fairly soon after Lat $B$ influx, whereas the dissipation of hotspots was a gradual process taking place over tens of minutes. At the same time, spine morphology also changed. Spines grew shorter and smaller, and spine heads became dimmer. The time course of the morphological changes paralleled that of the slow hotspot dissipation. We 
Spine lengths

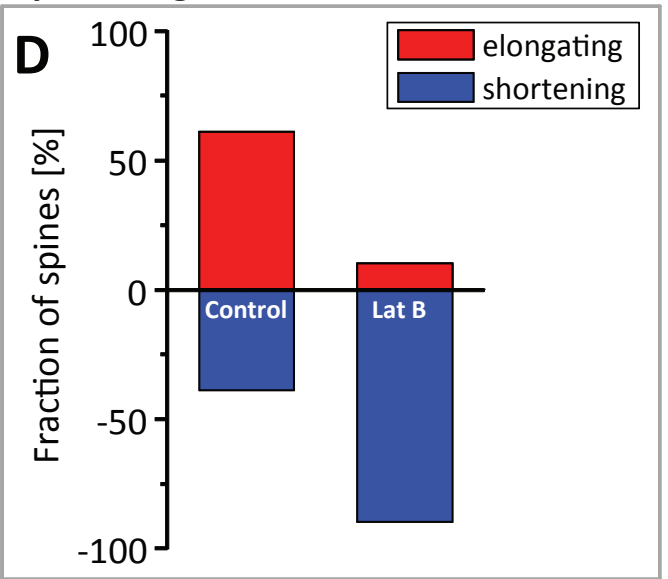

Spine brightness

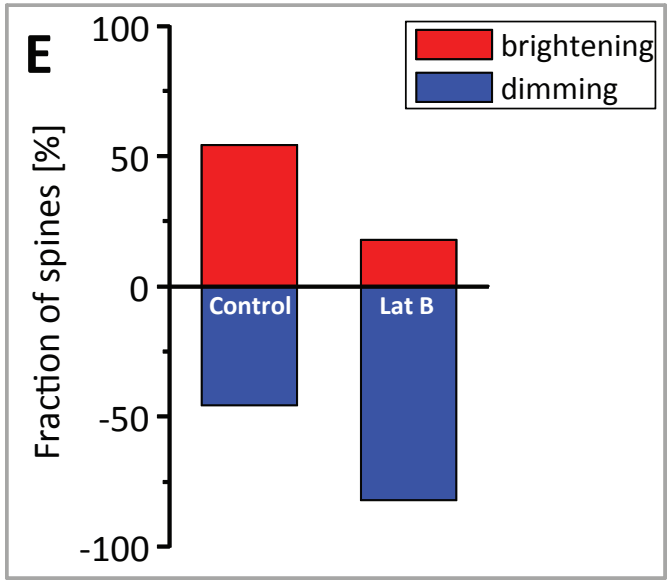

Figure 4.3. | Ratio of dendritic spines responding to Latrunculin B treatment compared to unstimulated conditions. The changes in length and brightness of dendritic spines were measured in time-lapse image series for both unstimulated (control) conditions and LatB treatment. (D) The ratio of dendritic spines that either lengthened or shortened after comparable amounts of time being exposed to regular ACSF (control, $\mathrm{N}=108$ ) or LatB treatment $(\mathrm{N}=39)$. Spine length was defined as the curvilinear distance from the spine base to the tip of the spine head. (E) Ratio of dendritic spines that displayed a brightening or dimming of the spine head for both control $(\mathrm{N}=107)$ and LatB treatment $(\mathrm{N}=39)$ conditions. Spine head brightness was averaged over a small area surrounding the point of maximum intensity within the spine head.

quantified the changes by measuring the total spine length, as defined by the curvilinear length from the spine base to the tip of the head, and the brightness in the spine heads, as defined by the maximum pixel brightness, under both Latrunculin-exposed and untreated control conditions (Figure 4.3). In the control case, morphological fluctuations were bidirectional and fairly even, i.e. dendritic spines elongated and shortened in roughly even manner (a slight skew towards higher values was present), and the spine heads brightened and dimmed in a similar fashion. In contrast, after exposure to Latrunculin B more than $80 \%$ of the hotspots in the spine heads dimmed and more than $90 \%$ of the spines shortened (Figure 4.3). These changes could be quite drastic, with spines shortening and dimming by more than $50 \%$. The rate and magnitude of changes varied from slice to slice, however, and individual spines reacted differently to the treatment. After removal of the Latrunculin and after its immediate effects had run their course, we observed little 
or no more spontaneous movement or morphological changes of the dendrite: all spine motility remained apparently entirely suppressed, as long as the Latrunculin was not washed out and removed. This was tested for a period of over six hours. In one case we allowed the slice to recover for more than 1.5 hours after washing out the Latrunculin. Even though the morphology of the spines did not recover, the spine heads brightened slightly.

\subsection{Actin structures in dendritic spines}

The high resolution of our adapted nanoscopes revealed actin distributions and morphological spine features that were too small to be observed with diffraction limited optical microscopy, i.e. smaller than $\approx 190 \mathrm{~nm}$. Structures this small have previously been observed with electron microscopy, but only in fixed samples. In our living samples, the actin concentration inside dendritic spines was often too dense to distinguish any isolated actin structures within the general morphology of the spines. Nevertheless, local actin distributions could infrequently be observed in the spine heads, necks and, to a lesser extent, in the dendritic shafts.

\subsubsection{Spine heads}

Spine heads were generally very bright with a fairly homogenous Lifeact signal. Spine head sizes and shapes varied strongly all along the dendrite, with no apparent correlation between neighboring spines. Such spine head diversity has often been observed, ${ }^{115,131}$ but never with this level of detail in living brain slices. In fact, very few spine heads were truly round or oval-shaped. Most spine heads displayed at least some small irregularities in their morphology, such as flat edges, indentations, sharp points or even small protrusions or thin spinules (Figure 4.4).

Some general spine head shapes were more abundant, such as hollow cups or crescent shapes. ${ }^{132}$ Such cup shapes can occur when a synapse is strengthened and the associated dendritic spine expands the contact area with its presynaptic partner, thereby encompassing it. These cups could be very dainty, with cup rims that were thinner than $85 \mathrm{~nm}$. Yet, despite the small size, these cup rims were still comparatively bright and densely labeled, suggesting high concentrations of 

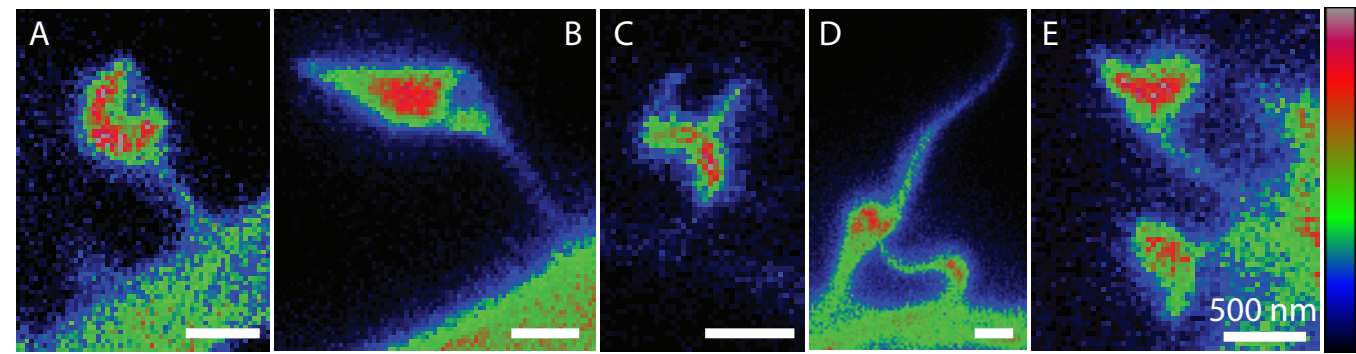

Figure 4.4. | Dendritic spine heads display very diverse morphologies, and exhibit small irregularities such as (A) indentations, (B) flat or pointy edges, (C) thin protrusions, (D) ephemeral spinules or (E) hollow cup-shapes.

filamentous actin supporting them (Figure 4.5). These cups could only be discerned if oriented suitably for optical sectioning, i.e. either parallel or perpendicular to the optical axis; in these cases they appeared as rings (viewed from above) or crescents (viewed from the side).
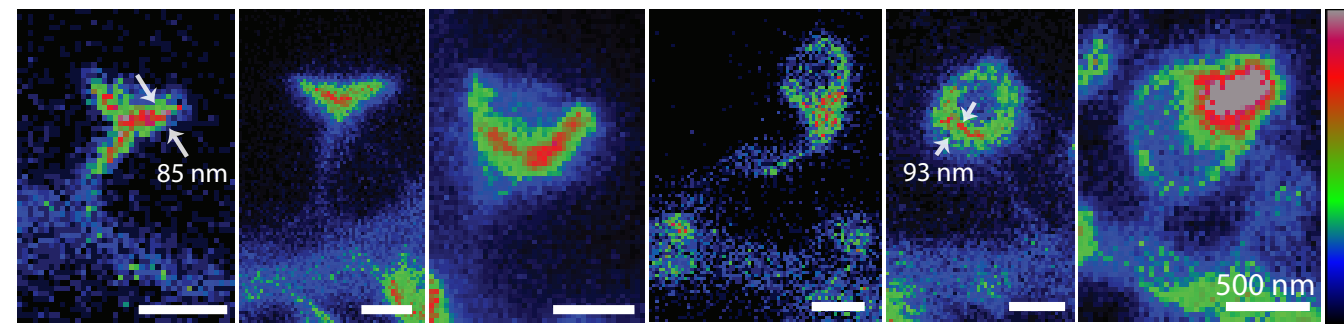

Figure 4.5. | Dendritic spine heads frequently exhibit cup-shaped geometries, which display bright actin labeling even when very small $(\leq 85 \mathrm{~nm})$. Depending on the orientation of the cup in regards to the optical axis, they can appear as crescents or rings.

Most dendritic spine heads exhibited fairly bright, homogeneous Lifeact concentrations. Yet a small subset of spines displayed distinct, irregular actin distributions in the spine heads. Of these, we most frequently observed asymmetric concentrations, or 'hotspots', along one side or corner of the head (Figure 4.6). These hotspots might denote especially high concentrations of short, highly branched actin filaments located at synaptic active zones, where they perform multiple roles, ${ }^{97}$ such as helping to stabilize and maintain numerous postsynaptic membrane proteins, ${ }^{133}$ as observed with electron microscopy. ${ }^{134}$ Distinct hotspots occurred especially in 
voluminous or lengthy spine heads, but not exclusively. Rarely did we distinguish multiple large or small hotspots in a single spine head (Figure 4.6).
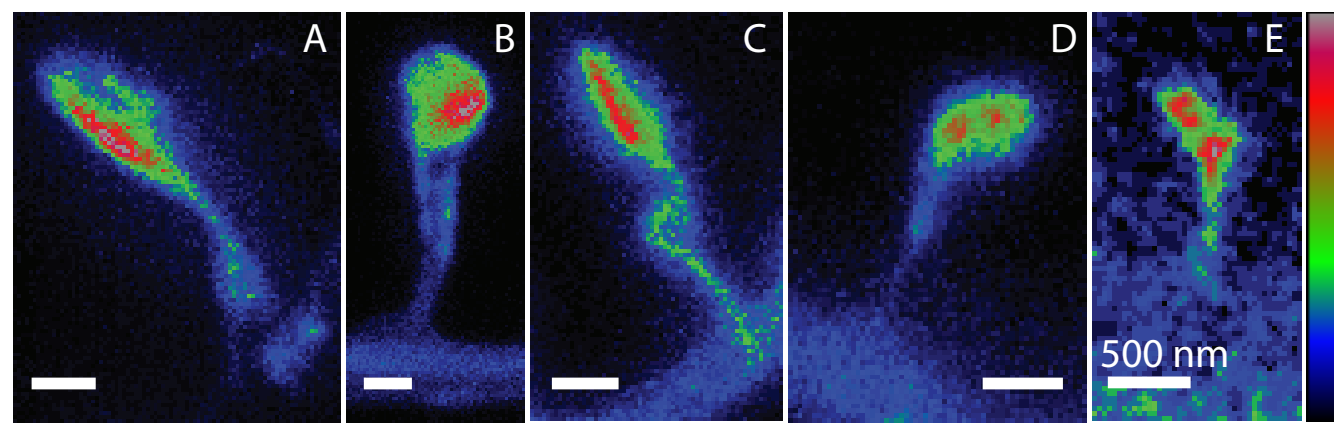

Figure 4.6. | Actin hotspots inside spine heads were visible with subdiffraction resolution. These hotspots appeared either as $(A, B, C)$ irregular concentrations on one side of a spine head, or as several small hotspots within the spine head's volume (D,E).

The actin sensitivity of the Lifeact label allowed us to discern between spines that appeared morphologically similar, but whose actin content hinted at wildly different functionality: on the one hand true filopodia or retracting spines devoid of actinenriched hotspots (and therefore an active synapse), on the other hand thin spines that lacked morphologically pronounced heads, but contained dense actin structures in the tip, suggesting an established postsynaptic density. Infrequently, we observed spine heads that were very large, yet nearly completely dark with no traces of actinenrichment (see also Figure 4.13 A,B). ${ }^{\mathrm{Vl}}$ Other recurring features of spine heads were dark areas, or 'holes', which were nearly devoid of fluorescence signal (Figure 4.7). These holes were diverse in shape and size, could be located anywhere within the spine head and could be observed to move around. Such holes might be signs of unlabeled organelles, such as the spine apparatus (a specialized form of smooth endoplasmatic reticulum), ${ }^{10}$ and have been observed in dissociated cultures. ${ }^{49}$

Individual spine heads contained well-defined corral-like actin structures (Figure 4.8). The actin filaments bordering these structures could be thinner than $100 \mathrm{~nm}$. These corrals were extremely rare in pyramidal neurons, but more frequent in certain types of non-pyramidal neurons that exhibited much fewer spines and a multitude of long actin cables running along the dendrites.

\footnotetext{
${ }^{\mathrm{VI}}$ Such dark, actin-devoid spines could be stable over tens of minutes or longer. Defocus was not the cause for the low brightness, as could be discerned by optical sectioning.
} 

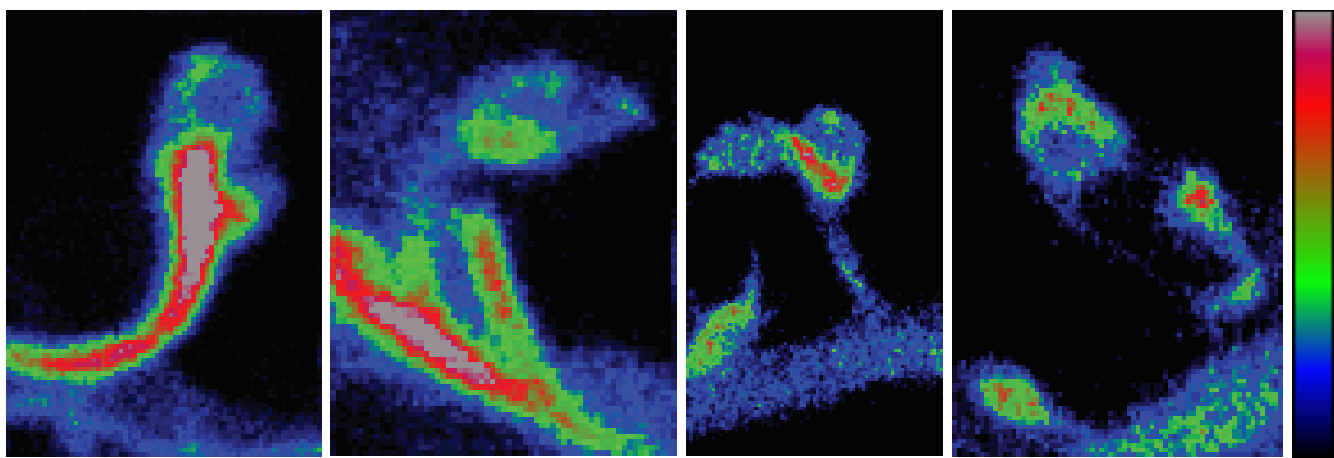

Figure 4.7. | Dark 'holes' in actin-enriched spine heads appeared in various shapes and sizes and could be located anywhere inside the spine heads.
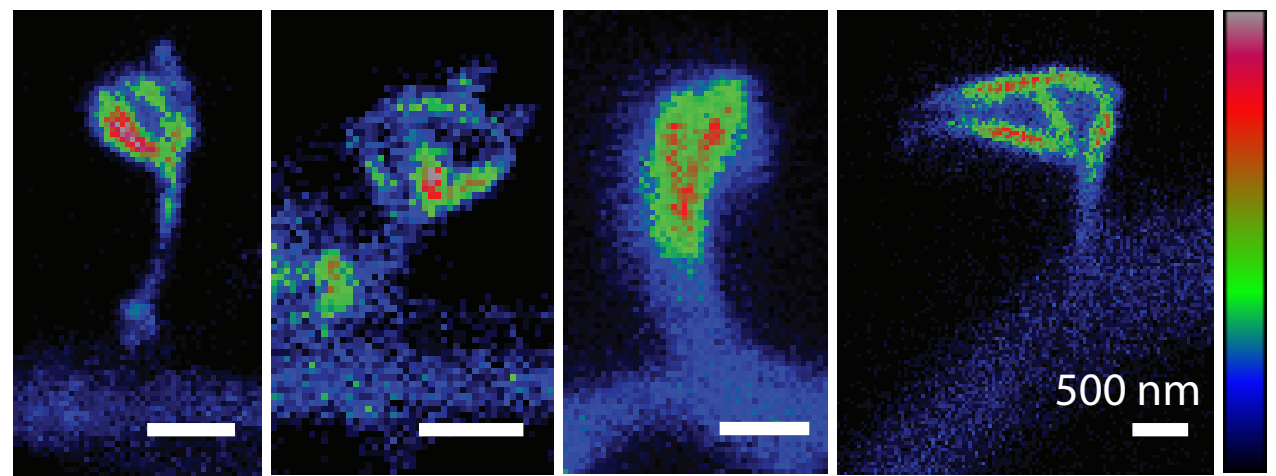

Figure 4.8. | Slender, corral-like actin formations in dendritic spine heads were observed infrequently, especially in CA1 pyramidal neurons. These structures were bordered by thin actin bundles, which could be less than $100 \mathrm{~nm}$ in diameter.

Most of the actin corrals in Figure 4.8 could unambiguously be assigned to dendritic spines belonging to CA1 pyramidal neurons. But in the case of dense, overlapping dendritic arbors, it was not always possible to tell the origin of every dendrite.

\subsubsection{Spine necks}

Dendritic spine necks themselves can be very thin and were frequently smaller than the diffraction limit of $190 \mathrm{~nm}$, especially in the CA1 hippocampal region, as discussed and measured in Section 3.3. Therefore, most of the distinct spine neck features we could observe were not resolvable by conventional optical microscopy. Many spine necks were labeled fairly homogenously and were typically brighter 
than the associated dendritic shafts but dimmer than the spine heads. This allowed very detailed measurements of the neck thickness, which varied considerably. The width of a spine neck fluctuated along its entire length - either smoothly undulating or tapering, or in abrupt bumps and constrictions (Figure 4.9).
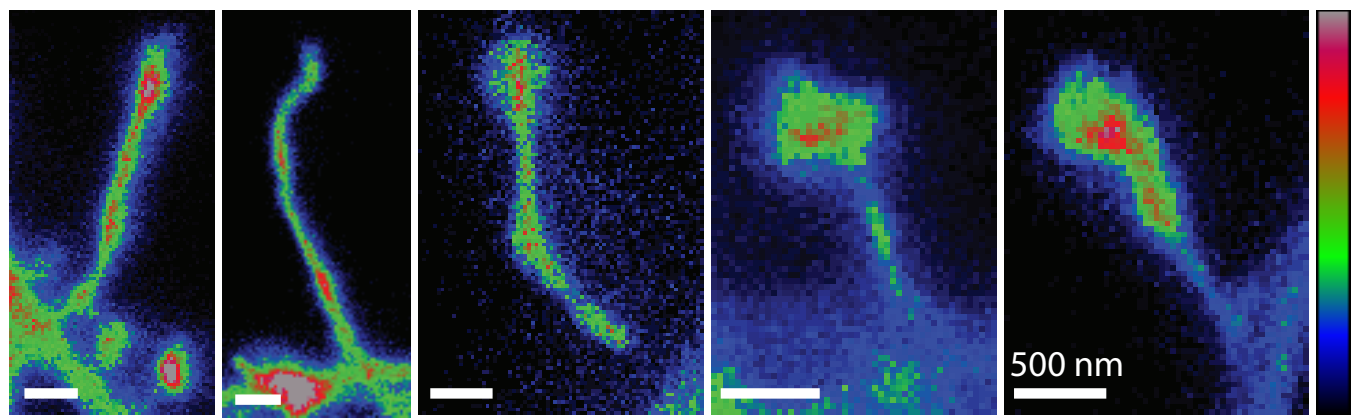

Figure 4.9. | The width of dendritic spine necks fluctuated along its length. Dendritic spine necks exhibiting a homogeneous labeling can vary considerably in width.

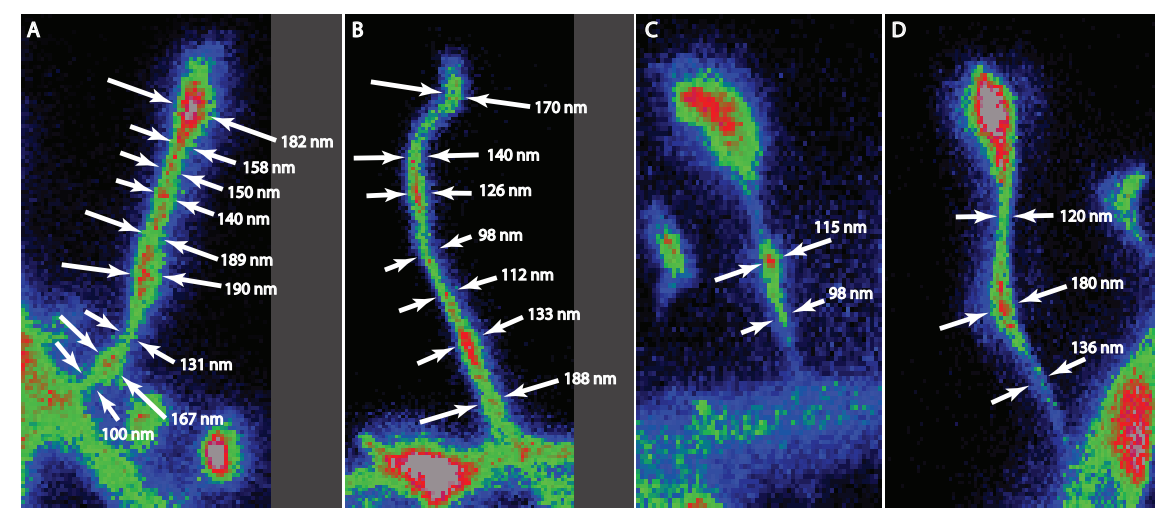

Figure 4.10. | Subtle fluctuation of spine neck widths can be measured with nanoscopic methods. Even when always thinner than the diffraction limit, dendritic spines can vary in width by more than a factor of two.

Even spine necks that were consistently thinner than the diffraction limit could fluctuate in width by more than a factor of two ${ }^{\mathrm{VII}}$ (Figure 4.10). Sometimes these varicosities coincided with bright actin hotspots, but they also occurred in homogenously labeled necks. Actin hotspots of different sizes and shapes were common in spine necks; they occurred at the tip, the middle or the base of the

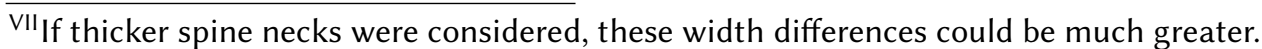


necks (Figure 4.11). The hotspots could be small enough (e.g. $<90 \mathrm{~nm}$ ) not to cause the surrounding spine neck to bulge visibly. In time-lapse experiments these actin hotspots could sometimes be observed appearing, dissipating or traversing the length of the spine neck. Most moving hotspots seemed to originate near the spine head and then travel away towards the dendritic shaft.
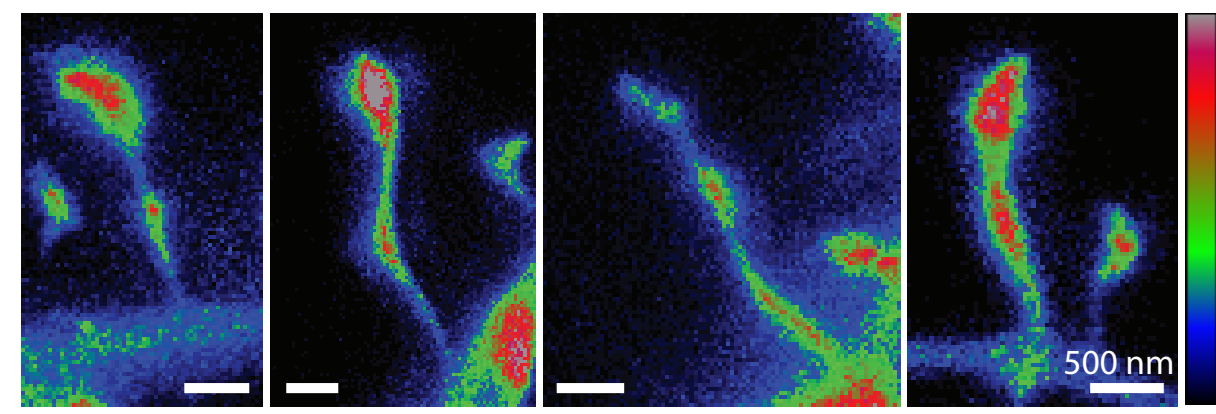

Figure 4.11. | Actin hotspots in spine necks can occur at every location within a spine neck. They can be seen to appear, disappear or traverse the length of the neck.

Infrequently, we observed spine necks with two actin bundles running in parallel along its length (Figure 4.12). The individual cables were between $120 \mathrm{~nm}$ to $155 \mathrm{~nm}$ in diameter, and were each located on the very outside of the spine neck, that is between $160 \mathrm{~nm}$ to $350 \mathrm{~nm}$ apart. These dual cables could be stable over tens of minutes (see also Figure 4.20 A).

The width of a spine neck was not directly correlated to the size of the spine head; even very large, bright spine heads could be connected to the dendritic shaft via thin, nearly actin-devoid spine necks (Figure 4.13). Conversely, very bright, thick spine necks could correspond to dim, wispy-thin spine ends. In general, spine necks were considerably dimmer than the associated spine heads, but could be equally

Figure 4.12 | Parallel actin bundles along the periphery of spine necks. Infrequently, twin actin bundles between $120 \mathrm{~nm}$ to $155 \mathrm{~nm}$ thick run alongside the inner periphery of a dendritic spine neck. These bundles have been observed to be stable over at least tens of minutes.
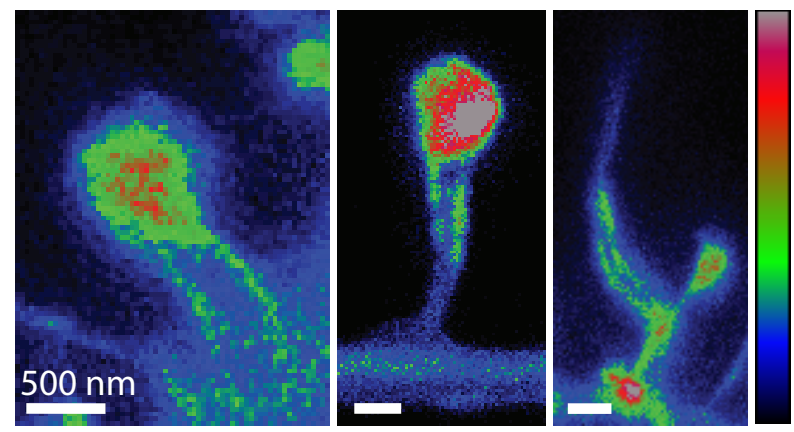
bright. But especially these spines undergoing large, abrupt morphological changes featured spine necks of equal labeling intensity as that of the spine heads.
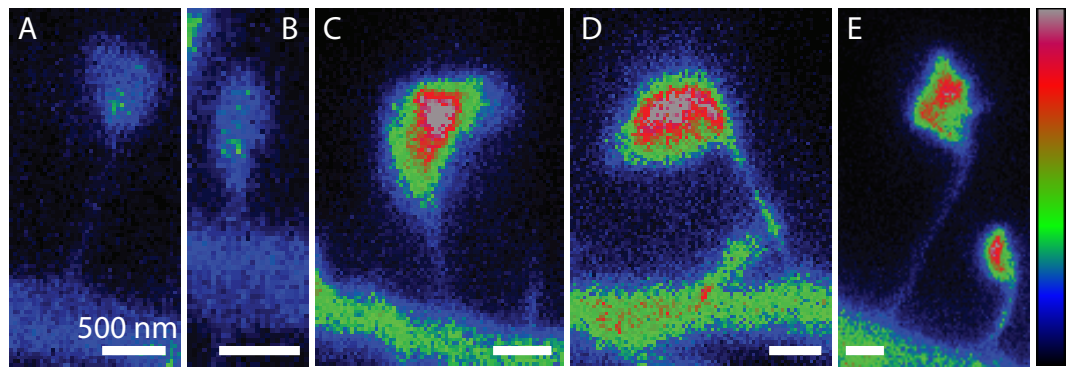

Figure 4.13. | Unusually dim spine heads and/or necks. (A,B) Some spine heads were not enriched in actin, but nearly completely dark. (C-E) Despite very large, bright spine heads, the associated spine necks are dim and extremely thin.

Anchor-like actin structures were frequently visible at the bases of spine necks, where the spines merged with the associated dendritic shaft. Some of these anchors were simply extensions of actin filaments emanating from the spine necks, either ending abruptly near the neck base (Figure 4.14 B,C) or continuing further in a straight or curved manner inside the dendritic shaft (Figure 4.14 A).

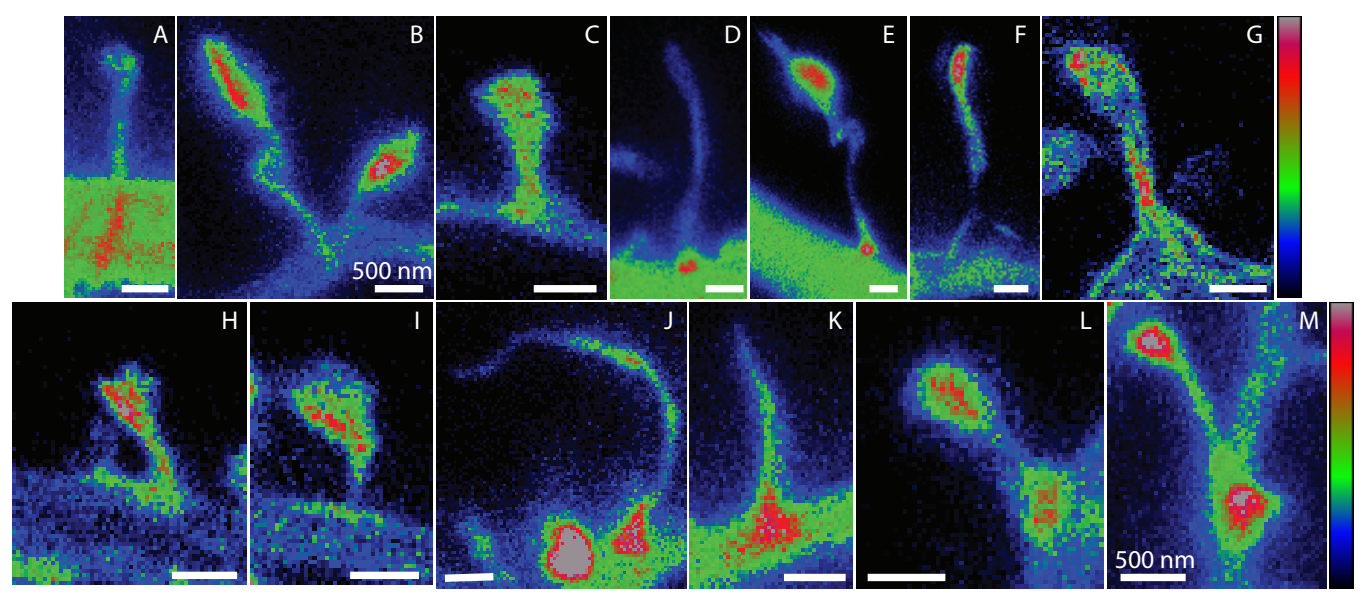

Figure 4.14. | Dense actin structures anchoring the spine neck at its base Actin hotspots can occur at the base of spine necks, where they adjoin to the parent dendrite. They can take the form of $(A, B, C)$ straight or branched filaments continuing from the spine neck, $(\mathrm{D}, \mathrm{E})$ dense hotspots, $(\mathrm{F}, \mathrm{G})$ thin, root-like filaments, $(\mathrm{H}, \mathrm{I})$ actin bundles perpendicular to the spine neck or $(J, K, L, M)$ delta-shaped actin concentrations. These actin structures can be as bright as the actin-rich spine heads themselves. 
Other possible configurations were small, dense hotspots directly at the neck base (Figure 4.14 D,E), several root-like filaments extending in various directions in the shaft (Figure $4.14 \mathrm{~F}, \mathrm{G}$ ), or thin, extended bundles running along the periphery of the shafts, perpendicular to the adjoined spine neck (Figure $4.14 \mathrm{H}, \mathrm{I}$ ). In particularly pronounced cases, these anchors took the form of delta-shaped cones that could rival the associated spine head in brightness (Figure 4.14 J,K,L,M). Similar deltashaped actin distributions have been observed with electron microscopy, linking the dendritic spine to the microtubule bundles running along the dendritic shaft. ${ }^{134}$

\subsubsection{Dendritic shafts}

Inside the dendritic shafts we could also observe distinct actin structures and bundles. Frequently, long actin bundles traversed the length of the dendritic shaft, especially along the periphery (Figure 4.15). These bundles could either be continuous, or consist of intermittent, shorter bundles centered around the bases of individual dendritic spines. Typically, such bundles were either very abundant in a dendrite, or completely absent.
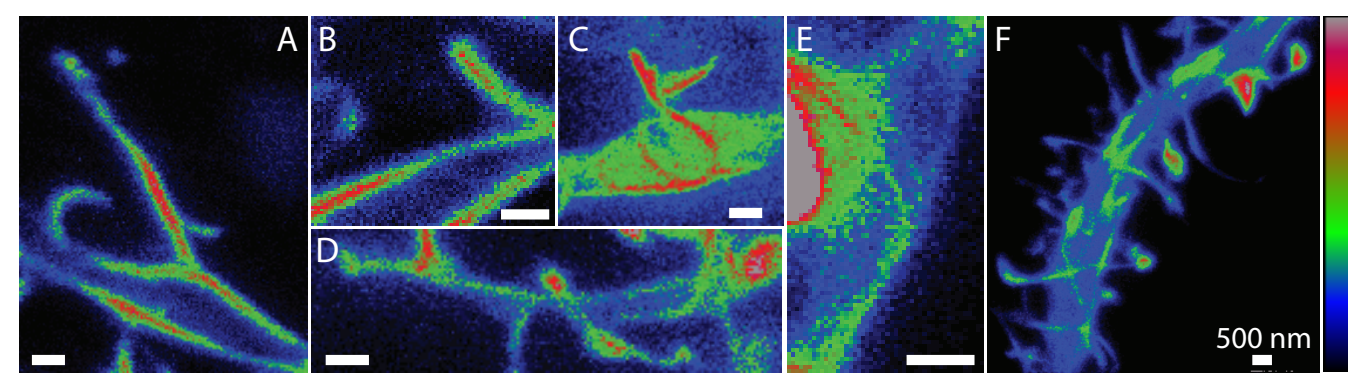

Figure 4.15. | Actin bundles inside dendritic shafts. Actin bundles of various lengths and geometries are present in dendritic shafts. Frequently they run along the periphery of the dendritic shaft $(A, B, D, E)$. The actin bundles can be connected to dendritic spines at the base $(A, B)$ and can even extend into or originate from them $(C, D, E, F)$.

Filaments extending from spine necks could be quite extensive and displayed various geometries: short or long, straight or tightly curved (Figure 4.15). It was difficult, however, to discern whether an observed actin structure was truly contained within a dendritic shaft, or, merely located within a dendritic spine that was immediately above or below the shaft. For our STED setup with diffraction-limited resolution along the optical axis $\left(\Delta x_{z} \geq 450 \mathrm{~nm}\right)$ this was possible only in the case of 
thicker dendrites, which were well contained in the imaging volume. Using the RESOLFT setup with improved three dimensional resolution, actin structures in the dendritic shaft could be identified much more easily and examined in greater detail (Figure 4.16). ${ }^{\text {VIII }}$ These bundles were not present at the base of every spine, even when they were in very close proximity to each other (Figure 4.16 A). Furthermore, these peripheral actin bundles could only be observed in Lifeact-stained samples, and not in neurons with a cytosolic labeling (Figure 4.16 C). ${ }^{\mathrm{XX}}$

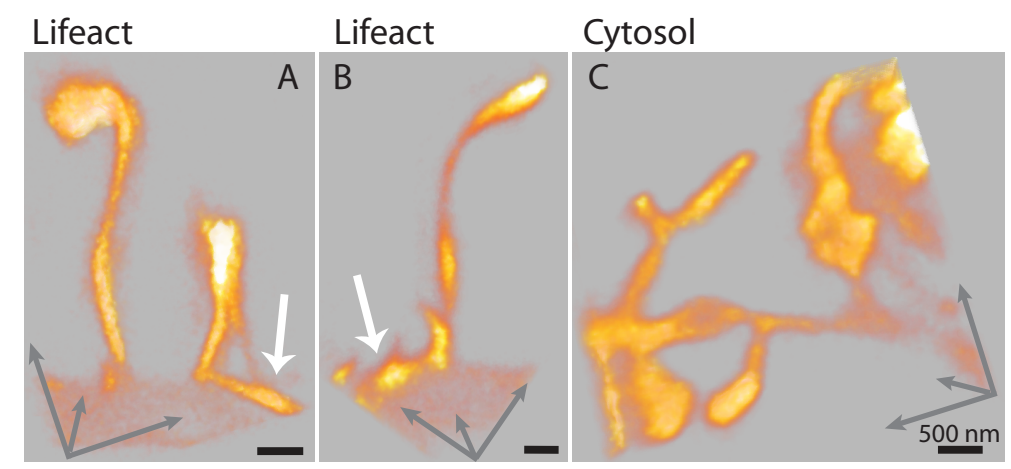

Figure 4.16. | Dendritic spines imaged with improved resolution in all three dimensions. By combining two de-excitation beams with different phase patterns, the microscope's spatial resolution can be improved in all three dimensions. With improved resolution along the optical axis, dendritic spines can be observed in greater detail. This is of special importance where the spine neck meets the shaft, when trying to localize thin structures within the thick dendritic shaft. Here actin cables can be observed adjoined to the spine neck, but inside the volume of the dendritic shaft $(A, B)$. These bundles are only present at some spines and cannot be seen when using a cytosolic label (C).

\subsection{Spine motility and spontaneous actin dynamics}

As could be expected from living specimens, the observed neurons were not static, but displayed various types of movement as well as morphological changes. These dynamic events took place on various different time frames. Outright morphological changes usually occurred over the course of tens of seconds and minutes. Such

\footnotetext{
VIII Such a two-donut configuration enabling improved three dimensional resolution is not limited to RESOLFT, but can be implemented with STED as well.

${ }^{\mathrm{IX}}$ This may sound trivial, but it excludes the possibility that these long, peripheral bundles were caused by large, unstained organelles within the dendritic volume, which merely left thin strips of labeled cytosol on the edges of the dendrite. Such dark organelles were observed frequently in dendritic shafts, but their appearance was distinctly different.
} 
morphological changes encompassed spine heads deforming and increasing or decreasing in size (Figure $4.17 \mathrm{~A}, \mathrm{~B}, \mathrm{C}, \mathrm{D}$ ), spine necks lengthening, shrinking or simply shifting (Figure 4.17 E), as well as entirely new structures, such as fine spinules or filopodia, erupting from existing structures (Figure 4.17 F).

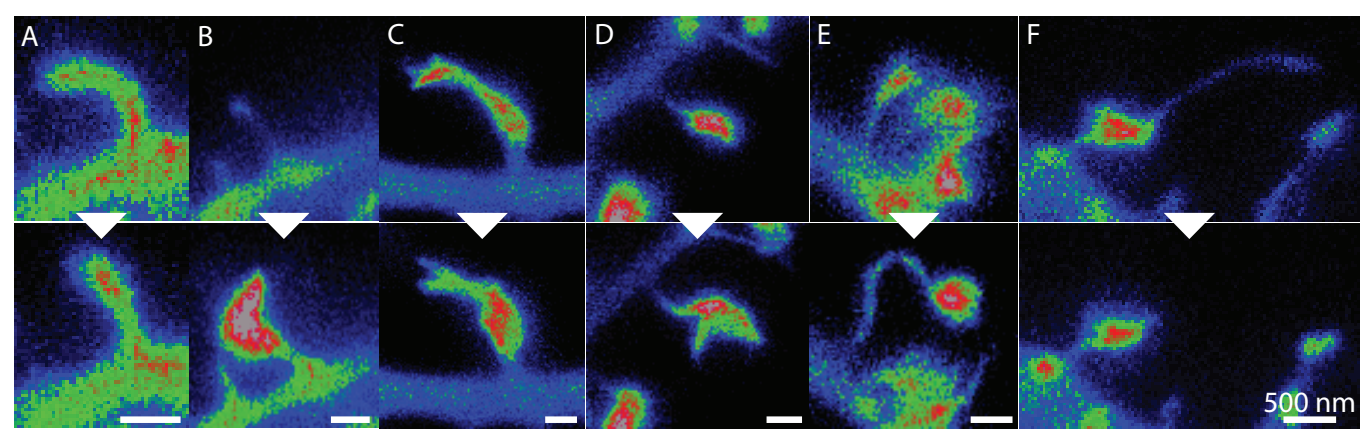

Figure 4.17. | Dendritic spines undergo strong morphological changes in short times, such as (A) shrinking, (B) growth, $(C, D)$ shape changes, (E) lengthening or (F) transient generation of spinules. These changes can take place in only a few minutes.

The actin structures described in Section 4.4 could also be observed to be in flux, such as actin hotspots appearing, disassembling or moving within spine heads, necks or dendritic shafts (Figure 4.18). Some of these changes were transient, and disappeared as fast as the occurred.
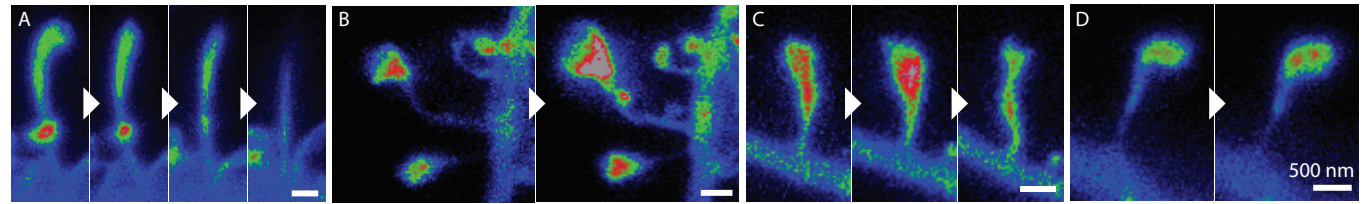

Figure 4.18. | Dendritic actin structures rapidly assemble and disappear. Actin hotspots in spine necks can (A) disperse or (B) appear abruptly, just as (D) in spine heads. (C) Actin agglomerations that are expelled from the spine head can appear in the neck as hotspots.

Movement was commonplace, such as individual spines drifting in and out of focus, shifting in regards to each other, or entire stretches of a dendrite twisting and shifting gradually. More permanent changes and movement on a larger scale could be observed as well, typically on the time scale of minutes to hours. These lasting changes could occur spontaneously or be a reaction to a deliberate, extrinsic 


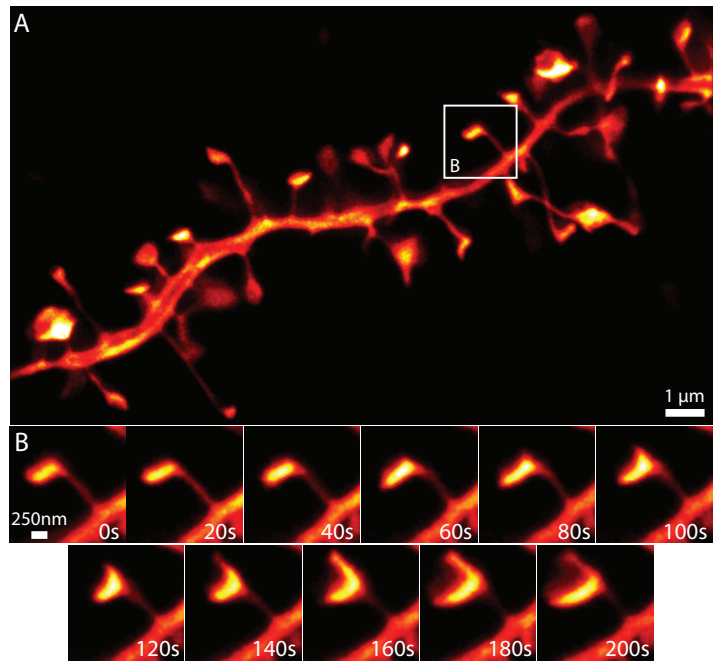

Figure 4.19| Spontaneous morphological changes of dendritic spines. (A) STED overview image of a dendrite from a CA1 pyramidal neuron labeled with cytosolic EYFP. This dendrite was imaged repeatedly every $20 \mathrm{~s}$ for over four minutes. An individual spine undergoes spontaneous morphological changes, completely changing its spine head shape from small and squareshaped to a much larger cup-shape in three minutes.

stimulus. Faster, more transient movement became apparent when imaging much smaller areas, usually containing only a few spines every couple of seconds. Some dendritic spines displayed very fast motility, similar to jiggling or delicate structures swaying gently in water. But not all dendrites exhibited motion or change. Entire segments of spiny dendrites could be near motionless over hours, not showing any lasting changes or movement that was visible when imaged every couple of minutes.

\subsubsection{Rapid morphological changes}

Most dendritic spines were fairly stable over the course of several hours, during which they retained their overall shape and size. But individual spines underwent very drastic shape changes in very short times (Figure 4.19).

Even after being stable for hours, spines could change completely in the span of tens of seconds to a couple of minutes. Spine head growth could be almost explosive, whereas head shrinkage tended to be much more gradual. Subtle changes of actin concentration could be observed in dendritic spines, such as multiple small hotspots $(\approx 240 \mathrm{~nm}$ ) in a spine head appearing and disappearing (Figure 4.18), or spine necks and heads gently undulating over time (Figure 4.20 B). Most dendritic spines that underwent rapid growth displayed very bright Lifeact signals wherever the change took place, suggesting that actin plays a major part in these rapid morphological rearrangements. ${ }^{117,118}$ 


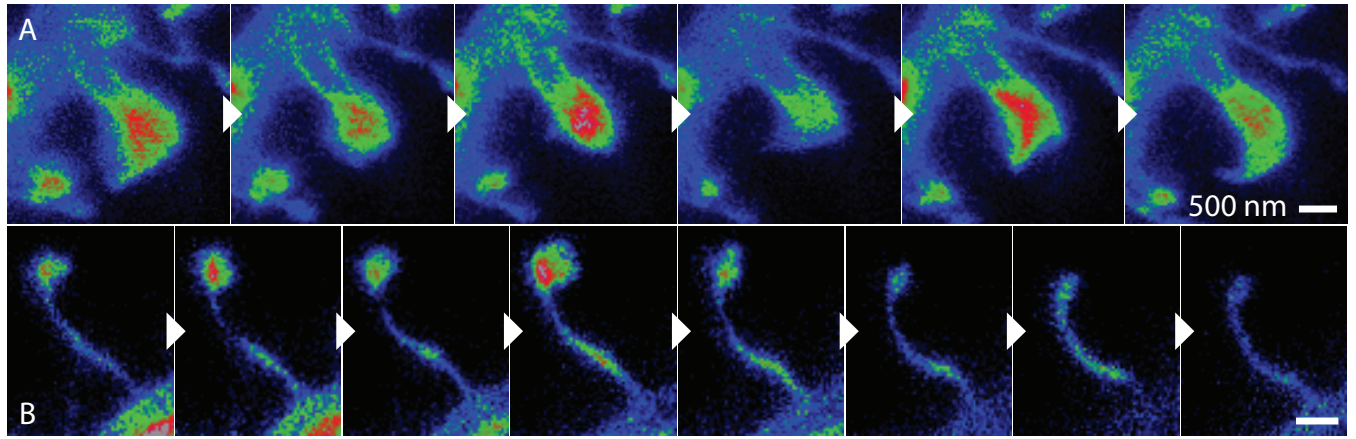

Figure 4.20. | Spine necks fluctuate less than spine heads. In general, spine heads displayed higher motility than spine necks and the actin structures therein. (A) Parallel actin cables in one spine neck are stable over tens of minutes, while the spine head morphology changes gradually. (B) The width and actin concentration in a spine neck changes slightly, but the overall shape remains stable. All the while the associated spine head grows and then shrinks by several hundred percent.

Microglial cells were also observed to undergo rapid changes. ${ }^{\mathrm{X}}$ In the brain microglia can take on very different forms, depending on the active role they are playing at the time. In our slices, we observed stationary microglia extend small thin protrusions into the surrounding area, probing for certain activation signs emanating from the neighboring tissue. These extensions could be very thin $(<100 \mathrm{~nm})$, and move quickly over tens of seconds to minutes (Figure 4.21). The width of these processes changed thickness rapidly, and extensions constantly merged with neighboring processes or split into two. This merging and splitting created clearly visible 'wandering intersections' that could be followed over several minutes.

\subsubsection{Fast motility and movement}

Even though most dendritic spines were fairly stable and maintained their general morphologies over long time periods, they could not be described as static. When observed on a very fast time scale (seconds), dendritic spines could be highly dynamic and prone to very fast movements and fluctuations of size and shape. Mostly, these dynamics were ephemeral, i.e. spines that deformed or moved rapidly returned to their previous form and position. To observe this, we recorded fast series

\footnotetext{
${ }^{\mathrm{X}}$ To observe microglia, we prepared hippocampal brain slices from a transgenic mouse strain expressing cytosolic EGFP in microglia.
} 


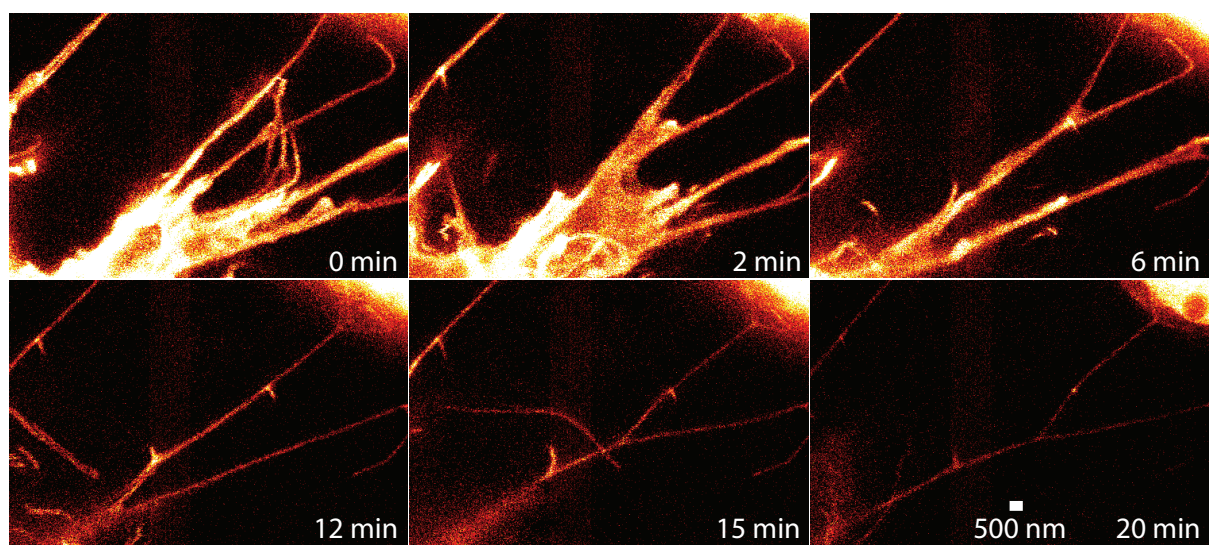

Figure 4.21. | Movement of branching processes on the periphery of activated microglia. EGFP labeled microglia from transgenic mouse strains were observed in hippocampal brain slices. Even in their amoeboid, activated state they extend thin processes into the surrounding area, probing for (chemical) signals. These processes could be very thin $(\leq 105 \mathrm{~nm})$, and were in a constant state of flux. They broadened, thinned, split into two or merged with neighboring processes in a matter of minutes or less.

of images, both with STED and RESOLFT using Lifeact-EYFP and Lifeact-Dronpa-

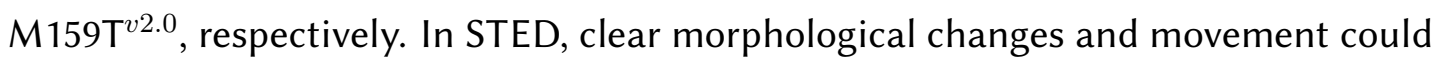
be observed on very fast time scales (Figure 4.19). This rapid imaging could not be maintained for too long, however, before bleaching became problematic and phototoxicity concerns arose. Also, the high amount of energy involved in the imaging process might be considered to influence or even provoke these movements. Therefore, we repeated these image series with RESOLFT microscopy, thereby alleviating the bleaching and high energy concerns. We observed stretches of spiny dendrites for several hours, to ensure that the dendrite remained healthy and to observe any long-term changes and movements. Intermittently, we recorded fast, continuous image series of individual groups of spines to track any fast dynamics; for these image series, one frame was taken every seven seconds for 40 frames (Figure 4.22). Over the course of several hours we observed only subtle morphological changes and movement. In the rapid images, however, individual spines display quick deformations, shift in regards to the surroundings and then return to their previous positions (Figure 4.22 B,C arrows). Most areas of the imaged dendrites remained stationary at any given time; but a stationary object in one image series could be highly motile in a later image series. Changes were 
most obvious at the spine heads, where they were more pronounced, but could be observed for spine necks as well. Actin cables inside dendritic shafts, however, seemed to be remarkably stable and remained stationary despite neighboring spines displaying rapid motility.

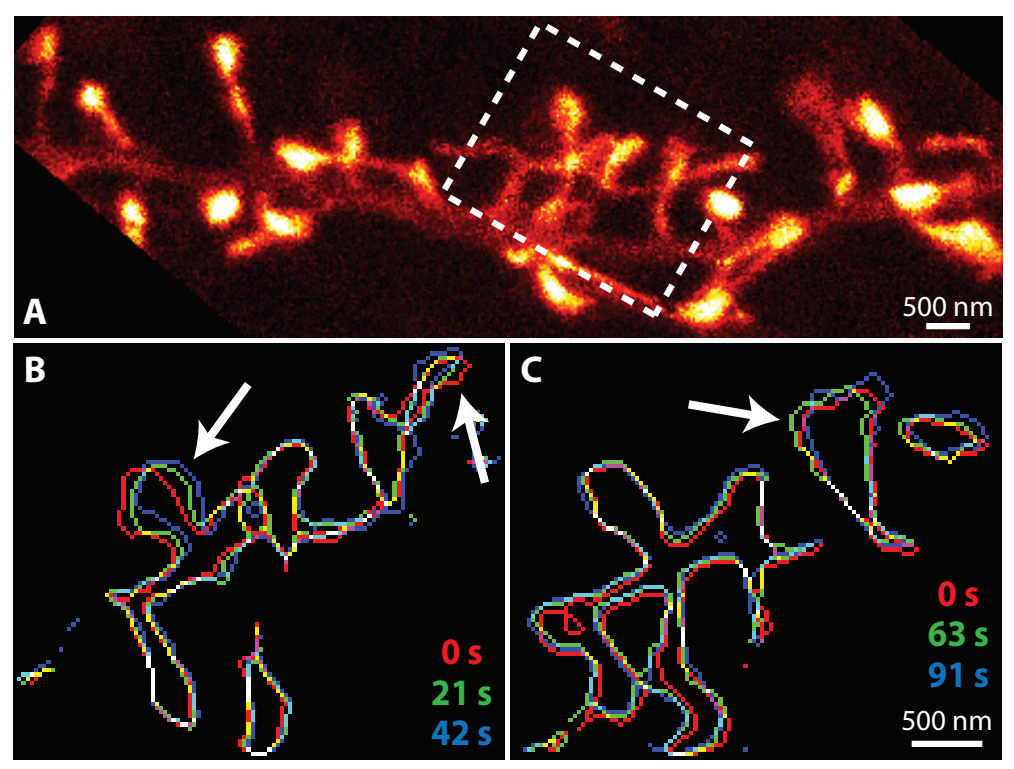

Figure 4.22. | Dendritic structures display rapid motility over tens of seconds. (A) Using RESOLFT nanoscopy a stretch of spiny dendrite was observed over several hours without exhibiting much large-scale movement. (B,C) But when observed on a much faster time scale ( $7 \mathrm{~s} /$ frame for 40 frames), individual spines can be seen to undergo rapid movement and slight morphological changes. Other parts of the dendrite are nearly perfectly still. The outlines of several spines from the boxed area in (A) were determined for several points in time and then superimposed, with each color (red, green, blue) depicting a different point in time. Other colors depict overlapping areas between the various points in time. Images were recorded using RESOLFT nanoscopy.

This rapid, but transient motility was not true for every dendrite, however. Exactly the opposite could be observed for other structures: little to no rapid motility, but distinct long-term changes of individual spines and even global twisting and shifting of the entire dendrite. Rapid motility and permanent changes could be observed on other dendrites. In conclusion, we could not observe any correlation between fast motility and propensity to long-term changes. Nevertheless, we observed the same type of rapid motility and dynamic changes using only low RESOLFT laser power as observed with the more powerful STED lasers. 


\subsubsection{Effects of heating}

We tested the effect of temperature on spine motility, net movement and overall rate of change. For this we performed RESOLFT imaging at low powers to reduce possible effects caused by strong laser illumination. At first, we observed brain slices at room temperature and looked for both fast and slow dynamics. Almost no spontaneous dynamic processes could be detected. Spines were almost completely static with no spontaneous long-term changes or fast motility detectable. It was possible, however, to elicit stimulated morphological changes using a chemical LTP protocol (as discussed in Section 4.6). Therefore, the dendritic spines were not intrinsically immobile at room temperature but retained the ability to undergo change. When brain slices were heated to $35^{\circ} \mathrm{C}$, the overall motility increased dramatically. Pronounced spontaneous changes or spine fluctuations were still rare, but far more frequent than at room temperature. Also, stronger movements, such as entire shifts and twists of the dendritic shaft could be observed.

\subsubsection{Continuous time-lapse imaging and cell viability}

In order to observe long-term changes occurring in brain slices under more physiological conditions, we need to be able to image these structures without disturbing or interfering with these delicate processes. We therefore scrutinized them for typical signs of (photo-induced) cell damage, such as dendrite blebbing, rapid and severe bleaching or dimming of dendritic segments, strong drifts within the sample, a sudden (local) increase in opacity of the brain slice, and unusual or collectively occurring morphological changes of dendritic spines. Furthermore, we watched out for signs that the imaging procedure was inducing or altering the observed dynamics in the brain slices, such as sudden deviations in the frequency or magnitude of the overall motility, movements and morphological changes, or differing motility in the imaged area and neighboring areas. Also, we looked for gradually increasing or decreasing motility over the course of the experiments, possibly correlated with the amount of laser exposure during imaging. The factors to consider here were laser power, average exposure time (depending on the pixel size and dwell time, frame size and number of optical sections) and deliberate imaging breaks. For STED imaging, if we maintained continuous, uninterrupted imaging of dendritic segments 
at high STED powers, we observed progressive bleaching of the dye, sometimes followed by eventual dendrite blebbing and dissolution of the observed structures. If imaging breaks were incorporated in between each stack or frame, the bleaching and consecutive photodamage could be greatly reduced or even eliminated. Imaging breaks of $30 \mathrm{~s}$ to $60 \mathrm{~s}$ were sufficient after single overview frames or several repetitions of smaller, rapidly acquired frames. For longer experiments, in which we were not interested in rapid motility or transient changes, we incorporated longer imaging breaks of several minutes $(2 \mathrm{~min}$ to $10 \mathrm{~min})$ to reduce the possibility of photo-induced effects and allow the dendrites time to recover from phototoxic stress and the label to replenish. With these imaging routines, we imaged dendrites over 6-8 hours without any visible detrimental effects; at the end of these time periods, spine motility and small changes could still be observed.

We could not detect any correlation between duration of an experiment and the likelihood of cell death. If cell death occurred, it usually heralded the collective demise of the neurons in the whole brain slice. Infrequently, individual neurons reacted to the imaging procedure and died within several minutes of original imaging. This occurred mainly for neurons exhibiting very dim labeling. For these neurons, however, there was no correlation between power used for imaging and cell death, as this even occurred for confocal imaging at very low powers. These neurons seemed to be declining in health, anyway, and were pushed 'over the edge' by the additional exposure during imaging. For healthy looking cells with good label expression such triggered cell deaths were not observed at all.

We tested the impact of laser absorption by irradiating unlabeled areas within the brain slices continuously using high STED powers. No signs of damage could be observed in the unlabeled areas, and no apparent effects were visible in adjacent areas with labeled neurons. Therefore, heating due to beam absorption seemed not to be of importance.

We further assessed the impact of laser illumination on cellular viability by imaging neurons for extended times with RESOLFT, thereby decreasing the involved laser intensities by five to six orders of magnitude. Here, we did not incorporate any deliberate imaging breaks. Instead, we observed larger areas continuously over several hours. An imaging area of $10 \mu \mathrm{m} \times 20 \mu \mathrm{m}$ with $25 \mathrm{~nm}$ wide pixels required 
160 s exposure. Typically, we acquired 5 optical sections (frames) per stack, which (at this acquisition speed) amounted to a total of 45 frames every two hours. Smaller areas could be recorded much faster (e.g. $4.2 \mu \mathrm{m} \times 3 \mu \mathrm{m}$ in seven seconds, see also Figure 4.22), but we limited the number of recorded frames without interruption to 100-120 frames. For such fast imaging series, we noticed slight bleaching (which would eventually replenish), but no signs of photodamage. Typically, we observed a bleaching of $10 \%$ after 40 frames. Overall, we observed with RESOLFT the same dendritic motility as observed with STED, which did not change with experiment duration.

\subsection{Long-term potentiation}

Two hotly debated topics in synaptic neuroscience are the questions how dendritic spines react to stimuli, and what the precise function of the spine neck is. Whereas the length of the spine neck and the approximate size of spine head can be measured with diffraction-limited microscopy, the width of spine necks is too small to be resolved properly. Therefore, we addressed these questions by observing the response of dendritic spine necks to stimuli. For this, we used a chemical longterm potentiation protocol (chemLTP, or just LTP), with which synapses on a very broad scale are stimulated. ${ }^{51}$ The chemical LTP was administered by interrupting the suffusion of regular ACSF and instead washing a potentiation medium into the sample chamber, which contained elevated amounts of $\mathrm{Ca}^{2+}$, reduced amounts of $\mathrm{Mg}^{2+}$, and the potassium-channel blocker tetraethylammonium chloride (TEA-Cl). After 7-9 minutes the potentiation medium was washed out again by the regular ACSF. STED images were recorded before, during and at subsequent times after bath application of the potentiation medium. In the case of successful stimulation, the associated synapses undergo long-term potentiation, a process in which the strength of the synaptic connection is enhanced. In our measurements we focused on spine necks that were smaller than $250 \mathrm{~nm}$, and therefore mostly smaller than the diffraction limit. We imaged dendritic spines every couple of minutes for half an hour or longer, either in stimulated brain slices that were suffused with the chemical LTP buffer or in untreated brain slices for control purposes (Figure 4.23). 

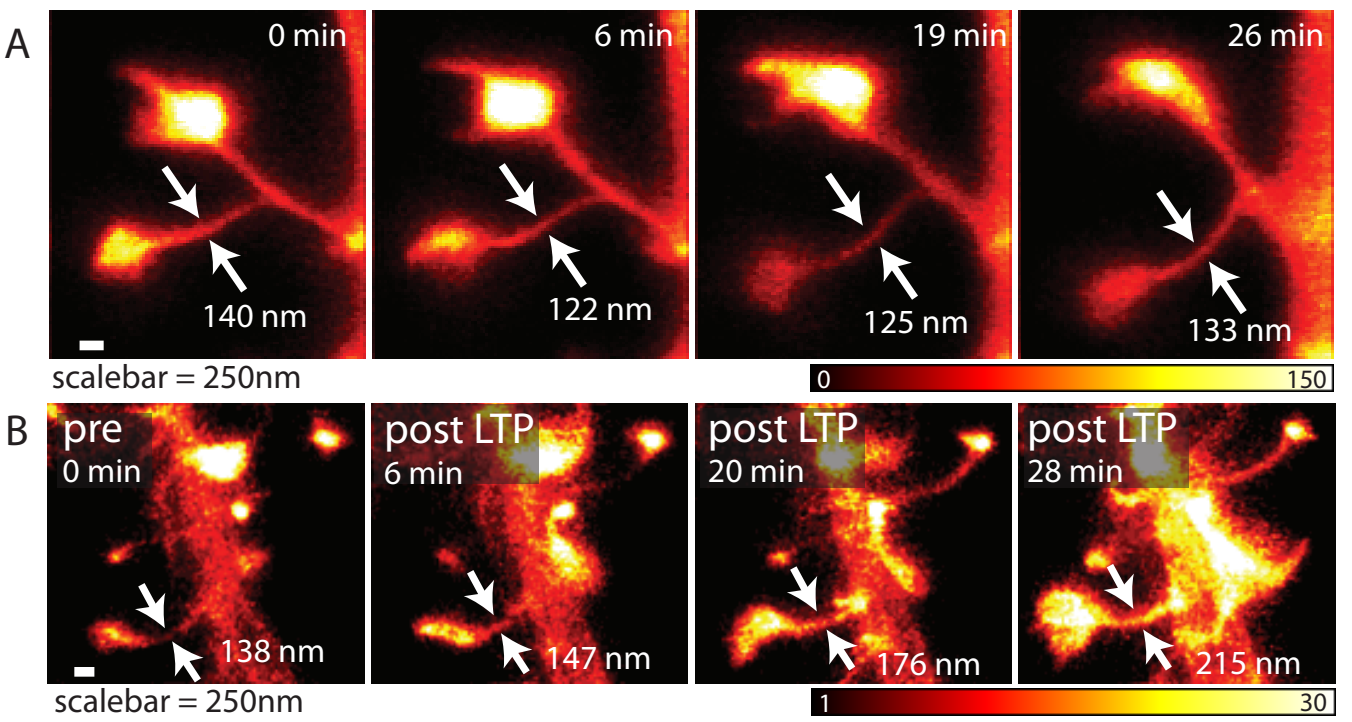

Figure 4.23. | STED imaging of postsynaptic morphological plasticity induced by chemical LTP Dendritic spines were observed repeatedly for 30 minutes (A) under unstimulated control conditions and (B) before and at subsequent times after a chemical LTP stimulation. Spine neck diameters were measured in each frame (indicated by arrows).

Under unstimulated conditions we observed strong bidirectional changes in spine neck diameter. But because these changes were bidirectional, they did not significantly alter the mean value across the spine neck population $(p=0.17$, paired $t$-test, $\mathrm{n}=34$ ); this can be expected for a steady-state system of course. On average, a dendritic spine changed its diameter by $24 \pm 5 \% \mathrm{XI}$ in either direction without having received an external stimulation. Next, we monitored the neck diameters after the neurons were stimulated to induce synapse strengthening. Again the spine necks displayed bidirectional fluctuations, but this time they were heavily skewed towards thicker neck values. On average the spine necks became wider by $28 \%$ (from $107 \pm 4 \mathrm{~nm}$ to $131 \pm 6 \mathrm{~nm}$; mean $\pm S E M$; $p<0.001$, paired $t$-test, $\mathrm{n}=41$ ). This encompassed both an increased number of spine necks that thickened, as well as a larger magnitude of changes (Figure 4.24). These strong changes were induced $\approx 5-30$ minutes after influx of the potentiation medium, after which they slowly stopped. Afterwards the induced changes were maintained for several hours (see also Figure 4.25). Slight fluctuations and movements were observed after this 30

\footnotetext{
${ }^{\mathrm{XI}}$ mean \pm standard error $(\mathrm{SEM})$
} 
minute period, corresponding to the previously discussed spine motility of live neurons (Section 4.5). The LTP-induced morphological changes took place over several minutes, i.e. far slower than the acquisition time of one image frame (11s). We did not observe any motion artifacts, such as the displacement of consecutive lines, which would have hinted at movement faster than the acquisition rate.
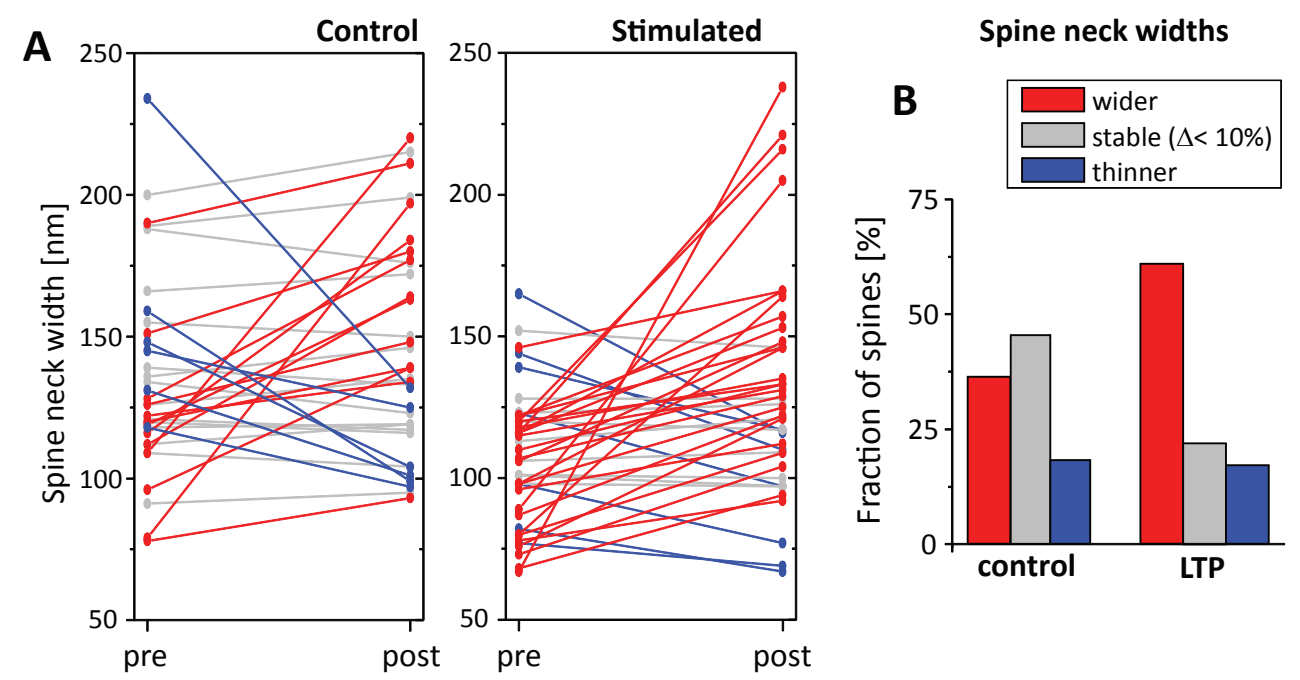

Figure 4.24. | Changes in spine neck diameter under control conditions and after LTP stimulation (A) Spine necks were measured before addition and after subsequent removal of the potentiation component (LTP) to the ACSF (see Section 2.4.4), or at comparable time points for control ACSF suffusion. Spine neck growth is shown in red, shrinking in blue and changes $<10 \%$ in gray. The change in neck diameters over the entire population was not significant for the control experiments, but increased strongly after LTP stimulation (paired two-sided $t$-test, $\alpha$-error $=0.05$; control: $\mathrm{p}=0.17, \mathrm{n}=34$; LTP: $\mathrm{p}=0.00096, \mathrm{n}=41$ ). We checked for normal distribution by examining the residuals and performing a Lilliefors (modified Kolmogorov-Smirnov) test. The homogeneity of variances was assessed with boxplots and performing a Levene test of homogeneity. (B) Ratio of dendritic spines exhibiting a measureable change in spine neck diameter after 10-30 minutes following pure ACSF suffusion (control, $n=74$ ) or chemical LTP treatment ( $\mathrm{n}=64$, right).

In some of the observed dendrites, the induced changes to dendritic spines were quite severe; spines grew massively in size, and the brightness of the entire segment increased strongly, as if the actin polymerization had increased. We therefore repeated these stimulation experiments with the low-power RESOLFT microscope, in order to rule out any influence of the imaging beam on the dynamics. The measurements and stimulation were performed analogously to the STED experiments, both for heated and room temperature samples. At both physiological 
and room temperature the LTP stimulus elicited obvious morphological responses, even though for room temperatures we had previously observed little to no spontaneous spine motility. With RESOLFT we observed the same responses of spine necks, which widened on average by $39 \%$ (from $143 \pm 5 \mathrm{~nm}$ to $194 \pm 6 \mathrm{~nm}$; mean $\pm S E M ; p<0.001$, paired $t$-test, $\mathrm{n}=24$ ). Again, the growth followed the same time line, with changes arrested after $\approx$ half an hour. Imaging for several more hours did not induce any further significant changes (Figure 4.25), nor was spine motility enhanced after stimulation than compared to before.

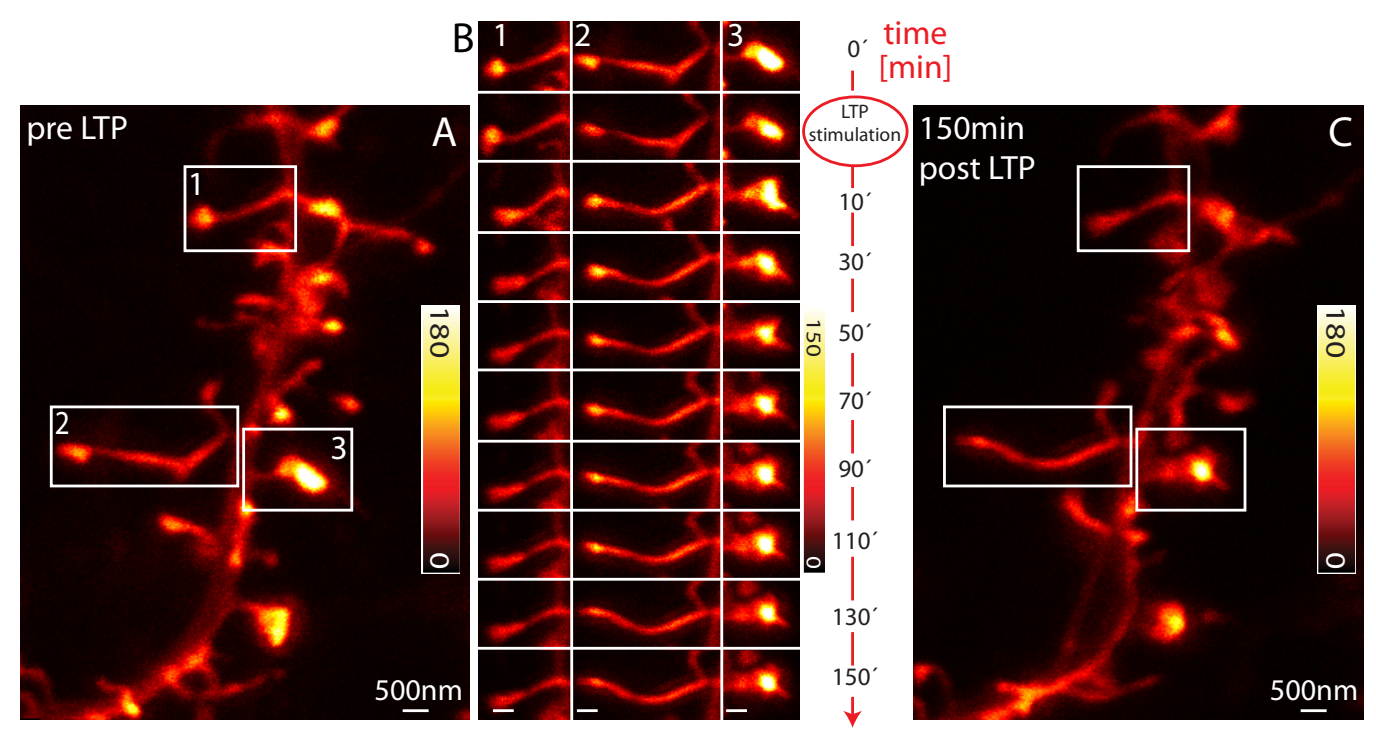

Figure 4.25. | RESOLFT imaging of LTP induced postsynaptic morphological changes. Spiny dendrites were imaged (A) before and repeatedly (C) after subjection to a chemical potentiation medium. (B) Clear morphological changes commenced following the chemical LTP stimulus, and continued for 30 minutes. After this time, the morphological changes were maintained and the spines returned to small-scale spontaneous movement and change.

We furthermore measured the length of spine necks and the average cross section of spine heads following the stimulation. We observed a slight shortening of spine necks after stimulation, and bidirectional fluctuations of the spine head cross section, which did not change for the overall population during RESOLFT measurements (see Figure A.3 in the appendix). With STED, however, we observed an overall increase in spine head cross section. So possibly the responses to the LTP stimulation were more pronounced when observed with STED than compared 
to RESOLFT, but higher statistics and more extensive measuring of a variety of dendritic properties (neck width and length, head size, shape and brightness) would be required to confirm this.

\subsection{Analysis and discussion}

The Lifeact-EYFP label proved to be a very simple and versatile labeling technique, albeit with some drawbacks. As stated in the original publication, Lifeact binds both to globular and filamentous actin. ${ }^{58}$ This became apparent when imaging transfected cells that expressed the Lifeact label. Dendritic spines, which are highly enriched in actin, were very bright compared to the much more voluminous dendritic shafts. The labeling was not homogenous: distinct hotspots, filaments and other structures were brightly visible. But in addition, there was a constant, homogenous background labeling of the cytosolic volume - either caused by Lifeact bound to G-Actin or unbound in the cytosol. This allowed us to trace the morphology and boundaries of the imaged dendritic structures, along with the detection of strong actin concentrations, using only one label.

\subsubsection{Morphological background label}

The obvious drawback, however, was the reduced contrast of actin structures due to the pervasive (morphological) background label. Dim actin structures might disappear entirely in the prevalent background. The label ambiguity towards F-and G-actin also meant that every apparent actin signal needed to be second-guessed, whether or not it was a true F-actin structure or something else. Unfortunately, there is no truly better alternative available. Phalloidin, the standard actin-labeling tool, permanently stabilizes F-actin structures, but is neither membrane permeable nor live cell compatible, as it irreversibly leads to cell death. By using fusion proteins of actin and a fluorescent protein, one would eliminate any signal coming from unbound Lifeact in the cytosol, but this protein would still be present in both F-and G-actin forms. Furthermore, without the rapid binding and unbinding of the Lifeact, bleached fluorescent proteins fused to actin could not be so easily replaced - only by completely disassembling and repolymerizing the actin structure. Possible solutions could involve utilizing a secondary label, which either helps to verify true actin 
structures, or otherwise maps the volume or outer surface of the dendritic structures. Actin-associated proteins that colocalize with filamentous actin structures would confirm actin structures. An unbound label in the cytosol or possibly bound to the outer membrane could show the true morphology of the dendrite, thereby allowing determination of structures invisible in the volume signal. Nevertheless, the duality of the Lifeact-label makes it a very versatile tool that is simple to use by fulfilling multiple roles at once.

\subsubsection{Bleaching}

The ability to replenish any bleached fluorescent proteins quickly was a huge advantage that aided both long-term and very rapid imaging. Because of this trait, we could image one location over and over again, completely unhindered by bleaching restraints. For STED, this was true if short imaging breaks were allotted, which needed only to be between $10 \mathrm{~s}$ to $60 \mathrm{~s}$ in most cases. Since bleaching was a much lesser problem for RESOLFT, no imaging breaks were required for long-term imaging. Even continuous, rapid scans of more than 100 frames in a few minutes were not only possible with RESOLFT, but produced high-contrast images.

Overall, our RESOLFT imaging was mostly unhindered by bleaching. With the replenishing Lifeact-label we could image small areas repeatedly every seven seconds for 40 frames with less than $10 \%$ bleaching. By incorporating longer breaks or simply by imaging larger areas, the bleaching could effectively be reduced to zero, as the label was completely replenished after each frame. To determine the actual bleaching, we imaged structures with a non-replenishing label. For this we expressed a fusion protein of Dronpa-M159T ${ }^{v 2.0}$ with HOMER1c in hippocampal neurons, again by viral transduction using a modified Semliki Forest virus. Homer1c is a scaffolding protein of the postsynaptic density, also known as Ves1-1L or PSD-Zip45, ${ }^{135,136}$ and can be found in dendritic spines. As a fusion protein, this label cannot bind and unbind (like Lifeact) and so lacks the ability to replenish. Furthermore, Homer1c does not undergo such strong turnover as actin. Any bleached fluorescent protein should therefore not be replaced quickly, allowing us to measure the bleaching. We imaged small areas containing Homer1c-labeled spines continuously, as above. After ten frames the bleaching was still only marginal 
(Figure A.4); after 40 frames the bleaching had increased to $50 \%$. Yet after 100 frames we still had enough signal to record a decent image. In conclusion, RESOLFT allows repeated imaging and observation for standard, non-replenishing labels. The replenishing Lifeact, however, negates the effects of bleaching almost entirely, thereby enabling near unlimited, continuous imaging.

\subsubsection{Depth penetration for RESOLFT imaging}

The same aberration correction techniques as demonstrated in Chapter 3 were applied to the RESOLFT nanoscope, i.e. a heated glycerol objective lens with correction collar. For the experiments, we imaged neurons that were in the second or third cell layer inside the slice, i.e. between $10 \mu \mathrm{m}$ to $50 \mu \mathrm{m}$ deep. These depths should suffice for the imaged neurons to be sufficiently embedded within intact tissue, and to have been undamaged by the cutting procedure and with most of their synaptic connections intact. The aberration compensation worked as before, allowing us to maintain the same spatial resolution throughout the slice (Figure A.2). It was apparent, however, that the z-donut was much more sensitive to aberrations than the $x y$-donut. This is because the helical xy-phase pattern created by the vortex phase plate was radially symmetrical, whereas the $\pi$-step phase pattern was not. Therefore, the sample aberrations needed to be compensated much more precisely to maintain the spatial resolution and avoid imposing severe aberrations on the beam PSF. If we had intended to image deeper inside the slice with RESOLFT, the much shorter scattering and absorption lengths of ultraviolet light in live tissue would have had to be taken into account. These would potentially reduce the feasible imaging depths within tissue as compared to STED microscopy. For very good z-resolution in high depths, therefore, a more sophisticated aberration reduction approach, as discussed in Section 3.4.2, would be highly beneficial.

\subsubsection{Low light exposure with RESOLFT}

Targeted superresolution methods, such as STED and RESOLFT, have certain advantages regarding exposure of the sample to light, as opposed to stochastic superresolution methods such as PALM and STORM. Because for targeted methods the light beam is rastered over the sample pixel by pixel, each location is only briefly 
exposed to laser illumination during each frame and is dark for the entire rest of imaging process whilst the beam is elsewhere. This provides natural imaging breaks, during which each location is granted some time to recover from any phototoxic stress and, when using the Lifeact label, the dye is given time to replenish. Combined with the inherent low light levels of RESOLFT, neurons could be imaged very gently with only little exposure to light. The main remaining consideration is the use of ultraviolet light for the on-switching of the RSFPs, which is surely not ideal for the viability of living cells. In our experiments the imaged neurons tolerated the UV light very well, suggesting that the light dose was small enough not to cause serious problems. Nevertheless, shifting the spectral range used in RESOLFT towards redder wavelengths can only be beneficial toward cell survivability. This could be accomplished either by using novel RSFPs which function solely using redshifted light, or by activating the currently used RSFPs using two-photon processes. A further prerequisite for novel RSFPs is the ability to switch fast enough, in order to allow the imaging of fast neuronal processes.

\subsubsection{Imaging speed}

The RSFP Dronpa-M159T ${ }^{v 2.0}$ used in these experiments for RESOLFT imaging proved to allow much faster imaging than demonstrated in previous RESOLFT implementations. ${ }^{55,137}$ The fast switching times enabled pixel dwell times of $400 \mu \mathrm{s}$, which allowed us to image (single optical sections of) entire dendritic sections $(20 \mu \mathrm{m} \times 10 \mu \mathrm{m})$ in under 3 minutes, small groups of spines $(4 \mu \mathrm{m} \times 3 \mu \mathrm{m})$ within seconds and individual spines in less than a second $(1.75 \mu \mathrm{m} \times 1.0 \mu \mathrm{m})$. These speeds are fully sufficient to observe even the rapid spine motility observed in STED experiments, which was on the scale of (tens of) seconds. The speed of RESOLFT recordings could be increased slightly by increasing the power of the off-switching beam, reducing the pixel dwell time and/or increasing the pixel size (typically $25 \mathrm{~nm}$ ); but such modifications in turn influenced the fluorophore stability ${ }^{\mathrm{XII}}$, signal strength and achievable resolution, respectively. For comparison, the typical imaging speed of our STED microscope was over ten times faster, requiring $11 \mathrm{~s}$ for a $20 \mu \mathrm{m}$

\footnotetext{
XIIThe slow off-switching step could be sped up by increasing the power of the off-switching beam, but this had two limitations. Higher powers could lead to strong non-linear effects that dramatically increased bleaching. Also, the switching speed saturated at certain powers, above which no further increase was possible.
} 
$\times 20 \mu \mathrm{m}$ frame with $30 \mathrm{~nm}$ pixels; typically, five optical sections per stack were recorded, resulting in just under one minute per $20 \mu \mathrm{m} \times 20 \mu \mathrm{m} \times 2.5 \mu \mathrm{m}$ stack. The achievable imaging speeds using this RESOLFT configuration with these fluorescent probes are physically limited by the time required for the RSFPs to switch from one state to the other. In our case, this is of the order of $100 \mu \mathrm{s}$, meaning that we can potentially speed up the process by a factor of four.

\subsubsection{Viral transfection}

The Semliki Forest Virus we used for transfection elicited fast responses of the affected neurons, which expressed the fluorescent label typically within 6-8 hours. The virus was neuron-specific, making it easy to transfect the desired CA1 pyramidal neurons. The viral CMV promoter induced very bright fluorescent signals, resulting in images with high signal-to-noise ratios. The speed and intensity of the transfection was so severe, that transfected neurons irrevocably died within 2-3 days of transfection. Therefore, in order to image neurons over several days, one would need to improve the cell health and survivability. For this, a slower, gentler virus, such as a Lenti or Adeno-associated virus, could be used. Unfortunately, Lenti viruses and, to a certain extent, Adeno-associated viruses are classified as S2 biological safety hazards, which would require significantly higher safety standards in both the optical and the wet laboratoires.

\subsubsection{Influence of optical recordings on neuronal health or dynamics?}

How sure can we be sure to what extent our imaging procedures influenced and disturbed the observed neural system? And whether or not the imaging itself caused serious photodamage? Electrophysiological measurements such as patch clamping or the use of voltage-sensitive dyes could ascertain whether neurons are still capable to respond to electrical or chemical stimuli. Such tests, however, are very invasive and cause physical damage to neurons by excising membrane patches, inserting needle electrodes or introducing toxic voltage-sensitive dyes. Furthermore, these methods would significantly increase the complexity of the experiment by requiring delicate measurements to be taken after (or even during) an imaging experiment. 
We can deduce some information on the health of our brain cells by looking for (the absence of) certain signals. When a neuron died in the experiment, its demise was usually heralded by a blebbing of the imaged dendrite. The blebbing dendrite underwent drastic local swelling and constricting, which lead it to appear as a lose chain of pearls. The blebbing was concomitant with severe rapid bleaching of the affected dendrite. So the absence of typical signs of cell death, such as rapid morphological changes, swelling, increasing tissue opacity, strong drifts, bleaching could be taken as indications that the surrounding tissue was still healthy. The continuing motility of imaged dendritic spines was also taken as a healthy sign, because such motility ceases upon suffering photodamage. ${ }^{113}$ As for signs of photoinduced effects of spine dynamics, we scrutinized for any unusual or changing behavior, such as collective responses from neighboring spines. We especially looked for any correlation to the light exposure and imaging duration. But in most (unstimulated) experiments we observed independent reactions and responses of neighboring spines and no changing dynamics or motility depending on the duration of the imaging. This would suggest that the imaging procedures did not influence the neuronal dynamics and that photo-induced effects were negligible. This was true for every RESOLFT measurement and for all STED measurements conducted with imaging breaks, which includes all the conducted and evaluated long-term experiments. It was possible to damage neurons by fast, continuous STED imaging, which would lead first to strong bleaching in the dendrite followed by an eventual cell-demise. The observed cell death was always accompanied by drastic morphological changes and effects. So the absence of typical signs of cell damage and death can be taken as strong indications (yet no full validation) that the imaged cell remained healthy. This, of course, could not give any clue as to the overall status of the brain-wide synaptic network, only as to the health of individual neurons.

\subsubsection{Application of potentiation medium or Latrunculin solution}

Experiments in which brain slices were exposed to drugs or different media elicited visible effects in about half of the measured slices. Consequently, either the neurons we observed in the brain slices were dysfunctional and/or unaffected by the drugs, or the drugs were prevented from reaching the neurons inside the brain slice in 
sufficient high concentrations. Of course, in each slice we could only observe a few dendritic segments; and only one segment could be imaged consecutively before, during and after drug application. After each experiment, we compared the imaged dendrites before and after drug influx. If any change or effect whatsoever was visible coinciding with the drug application, then the data was evaluated. If no neurons showed any response, the results were discarded.

\subsubsection{Plasma clot acting as diffusion barrier?}

We considered the possibility that the plasma clot, which covers the brain slice and is used in fixing the brain slice to the cover slip, might have hindered the diffusion of larger molecules, effectively shielding the underlying slice from the drugs. We tried modifying the composition of the plasma clot to make it less dense and more porous, but to no noticeable effect. We attempted to bypass the plasma clot entirely by cutting slits or puncturing holes into it, or by injecting the drug solution directly into the intracellular space using a micropipette. Unfortunately, this typically either compromised the viability of the slice due to extensive cell damage, or caused such strong movements, that it was impossible to objectively discriminate between druginduced changes and movements of the neurons and shifts caused by the injection process itself. The plasma clot might possibly be circumvented entirely by using different brain slice culture methods. ${ }^{99}$ This should make drug application easier and faster, and would allow the examination of thicker slices (see also Section 3.4.1). This would require a complete redesign of the setup, however.

\subsubsection{Long-term potentiation}

In the case of long-term potentiation (LTP), several conditions needed to be met for any response to be visible. The imaged dendrite had to belong to a healthy neuron, which would react to the TEA-Cl induced stimulus; it had to be embedded within an intact synaptic network with functional and active synaptic contacts; and the potentiation medium needed to accumulate in adequate amounts to elicit the potassium channel blocking. If only one of these conditions was not met, then no postsynaptic response could have been expected. 
How can we be sure that we truly induce LTP using our method? True proof would involve using a secondary method (such as local field potentials ${ }^{51}$ ) to verify the induction of the long-term potentiation. What we did observe, however, was that when we saw a response, it followed shortly after the influx of the potentiation medium and was completed in roughly half an hour. Before and afterwards we observed only fleeting changes and motility, even when imaging for hours. In conclusion, we observed a reaction to some sort of stimulus, following the application of a well-accepted stimulation protocol.

Our results show a clear thickening of dendritic spine necks in the time following the LTP stimulus. These changes are maintained at least for several hours afterwards and did not disappear again. Furthermore, the fraction of spines that undergo shrinking after the stimulus matches the fraction in the unstimulated control cases. It seems that this spine fraction remains unresponsive to the stimulation, possibly because the synaptic connection was already in decline and therefore lacked the ability to react. The increased amount of changing spines seems to have come from the fraction that possessed the capability to be strengthened, but which under unstimulated control conditions only displayed minor spine neck fluctuations. This interpretation is backed by the increased magnitude of the observed changes after LTP stimulation. Overall, we can for the first time report live experiments, in which the diameter changes of dendritic spine necks are measured on the same spine as they happen, and not by comparing average neck diameters measured in different dead, fixed samples that either were or were not treated. To truly confirm the postsynaptic response of spine necks after induction of long-term potentiation, we should acquire higher statistics and possibly verify these results using alternative brain preparations.

A more physiological approach to perform the LTP stimulation would involve an electrophysiological stimulation of individual neurons using precisely determined pulse sequences. In this way, we could stimulate individual neurons or at least restrict the stimulus to small areas, instead of stimulating every susceptible synapse in the entire brain slice. An even cleaner, yet much more difficult approach, would involve stimulating a neuron, whose axon forms a synapse with the observed dendritic spine. This way, we could stimulate the postsynapse directly by triggering 
presynaptic signals, which more closely emulates a true physiological stimulus. This approach would require dual labeling, however, (both the pre- and postsynaptic cells would need to be visible in different color channels) and at least one patch-clamp setup, if not two (again, one for the pre- and postsynaptic cells, respectively).

\subsubsection{Inhibition of motility and morphological changes by Latrunculin}

Would we have expected to observe a full recovery of the actin structures in the neurons that had been exposed to the actin-disassembling toxin Latrunculin? It can be argued that we observed the tentative beginnings of a recovery (in an individual case) several hours after washing out the toxin, but this was by no means conclusive. Other groups have reported full recovery of actin structures of cells that had been submitted to Latrunculin 24 hours after washing out the toxin. ${ }^{130}$ For us to observe a true recovery, we would have needed to extend our observation time from 3 hours to something much closer to 24 hours.

Should we expect an exposure to Latrunculin to consistently disturb the morphological structures of dendritic spines? There have been conflicting reports in the literature, some reporting clear morphological changes, whereas some report no visible changes whatsoever. ${ }^{138}$ Dendritic spines are highly enriched in actin, so dissolution of actin structures should influence the spines on some level. Especially young and immature spines, which are almost completely stabilized by actin filaments, should be affected in their morphology. As spines mature, however, the cytoskeletal network within becomes more complex as further components and stabilizing protein structures are added. For mature spines stabilized thus, dissolution of filamentous actin might not lead to visible morphological changes at all. It is true, that the neurons which elicited the strongest responses tended to be younger (several weeks old) and displayed more immature dendritic spines (such as long, thin spines, filopodia etc.). What should happen in all cases, however, is a freezing of all rapid spine motility, which has been shown to be driven by fast actin turnover and polymerization. Indeed, we did observe this and could maintain the diminished motility for hours, as long as sufficient levels of the Latrunculin toxin were maintained. 


\subsubsection{Fast spine motility}

The very fast motility that we observed in our experiments has been documented using other optical microscopy methods and a variety of different preparations, from dissociated cells ${ }^{49,112}$ over brain slices ${ }^{113}$ to brains in vivo; ${ }^{114}$ it has recently even been observed in vivo using a STED microscopy approach built using the same aberration compensation approach. ${ }^{53}$ We can therefore be fairly confident that this is in fact natural behavior that occurs in all healthy neuronal preparations. The similarity of the occurring dynamics observed using STED and RESOLFT nanoscopy, i.e. observed for irradiation intensities more than six orders of magnitude apart, suggests that the influence of our imaging procedures on the neurons was not very pronounced and possibly negligible. Our long-term STED experiments were all conducted in a 'gentle' manner by limiting de-excitation power and by introducing generous imaging breaks; other techniques or implementations, however, might exhibit stronger photo-induced effects if they do not take so much care to avoid tissue damage or even utilize more dangerous illumination schemes (such as very short pulses, as in two-photon microscopy). What is impossible to say, however, is whether even the very mild light dose of confocal or RESOLFT microscopy already disturbs the neural system. After all, there is at present no other method of directly imaging living samples in sufficient detail than optical microscopy.

\subsubsection{Influence of heating on spine motility}

In our RESOLFT imaging experiments we observed a clear increase of dendritic spine motility and unstimulated morphological changes at physiological temperatures of $35^{\circ} \mathrm{C}$ compared with room temperature $\left(21^{\circ} \mathrm{C}\right)$. This is not undisputed in the literature. Some reports confirm the increased motility ${ }^{113}$, whereas some experiments report to the contrary that spine motility is unchanged by temperature. ${ }^{139}$ In the latter publication, Korkotian et al. only examined the motility in five separate cases, with just a few time points each. Such low statistics might easily overlook strongly moving structures altogether if, as our experiments suggest, the fraction of highly motile dendritic spines is small to begin with. Then, even for a manifold increase of motility at physiological temperatures, the chance of finding a highly motile spine could be low. This is true especially for older, mature 
neurons, because the observed spine motility decreases exponentially with the age of the neurons. ${ }^{139}$ Nevertheless, we consistently observed more frequent and more pronounced motility and morphological changes at $35^{\circ} \mathrm{C}$. This increase in motility is not caused by increasing Brownian motion at higher temperatures, as has been verified by measuring the motility at temperatures above physiological levels $\left(45^{\circ} \mathrm{C}\right) .{ }^{113}$ There was no further increase of motility at these higher temperatures. Moreover, is seems that the physiological temperature range of $35^{\circ} \mathrm{C}$ to $37^{\circ} \mathrm{C}$ provides optimal conditions for neuronal spine motility, strongly increasing the propensity of dendritic spines to move and change shape. Even though we observed stimulated morphological changes both at room temperature and at physiological temperatures, the resulting effects of the stimulation might differ in magnitude and frequency of the changes, if they depend on the temperature. It seems prudent, therefore, to conduct all experiments that examine morphological change and motility at physiological temperatures.

\subsubsection{Differential motility of spine heads and spine necks}

All parts of dendritic spines were observed to be highly motile and prone to change, as could be observed in great detail using our STED and RESOLFT nanoscopes. Spine heads, however, underwent more pronounced morphological changes than spine necks, both in magnitude and in frequency (see Figure 4.20). The greater magnitude of changes is not entirely surprising, as spine heads were typically much larger than spine necks and displayed much higher actin concentrations, which has been shown to be the driving force behind rapid motility. Therefore, spine heads might be expected to be capable of more pronounced changes than spine necks. A further consideration is that the filamentous actin in spine heads is highly branched and can be turned over at very fast rates. Rapid expanding forces in all directions can therefore be exerted by increased actin polymerization in the spine head. The F-actin in spine necks has been shown to be highly linear and only slightly branched, which would result in far lesser opportunities for actin to generate expansionary forces directed towards the outside of spine neck. These differing motilities have been observed previously (also in cultured neurons using a stochastic superresolution technique). ${ }^{49}$ Yet without subdiffraction spatial resolution, the fluctuations of most spine necks would have been too subtle to have been noticed or measured accurately 
(see Figure 4.20). The low motility of actin bundles that we observed in dendritic shafts might be explained by the much slower turnover rate of actin in the shafts as compared to the necks or even the spine heads. ${ }^{118}$

Even with our high resolutions of $50 \mathrm{~nm}$ to $60 \mathrm{~nm}$ we could still only seldom resolve distinct actin structures in spine heads (such as in Figure 4.8 and Figure 4.6) although they are supposed to be full of filamentous actin. Assuming the actin filaments in the spine head are short and highly branched, as electron microscopy has shown, ${ }^{134}$ we would require isotropic resolution in the single-digit nanometer range to resolve these tightly-arranged three-dimensional bundles of actin. ${ }^{\text {XIII }}$ Therefore, most convoluted actin structures in the spines heads should appear as hotspots.

\subsubsection{Functional significance of morphological changes of spine necks}

Are the fluctuations of spine neck diameter that we observed truly relevant, if they are so small that we need superresolution to observe them? Minor fluctuations of spine necks widths in the range of several nanometers can safely be assumed to be transient and not to reflect any lasting changes in synaptic function. In the case of very thin spine necks, however, such as occur on dendrites of CA1 pyramidal neurons, even fluctuations that are below the diffraction limit may be of great importance. Fluctuations of $40 \mathrm{~nm}$ to $60 \mathrm{~nm}$ can potentially double or half the diameter of the very slender spine necks, which might well have strong implications for its function, such as changing significantly its electrical properties and/or altering its properties as a chemical diffusion barrier. Another reason why the motility and width fluctuations of spine necks might be of import, is the study of transport phenomena. It has recently been shown, for example, that microtubules can transiently invade a dendritic spine all the way to the tip to spine head; ${ }^{142}$ following such microtubule invasions, an increase of the PSD95 protein can be observed, ${ }^{\text {XIV }}$ suggesting import functions for molecular transport. ${ }^{143}$ Such invasions might be heralded by a widening of the spine neck. Moreover, in order to be able

\footnotetext{
$\overline{\mathrm{XIII} W h e r e a s ~ s u c h ~ h i g h ~ r e s o l u t i o n s ~(a t ~ l e a s t ~ i n ~ t w o ~ d i m e n s i o n s) ~ h a v e ~ b e e n ~ d e m o n s t r a t e d ~ u s i n g ~ S T E D ~}$ and ground-state depletion microscopy (GSD) of defects in nanodiamonds, ${ }^{140,141}$ the laser power involved was very intense and quite unsuitable for imaging live neurons.

${ }^{\mathrm{XIV}}$ For neurons exposed to brain-derived neurotrophic factor (BDNF) treatment.
} 
to observe the full range of dendritic spines, from large to small, and to accurately measure their morphological changes, very high spatial resolution is necessary, such as is provided by our nanoscopy setups.

\subsubsection{Is STED or RESOLFT better suited for neuronal imaging?}

Which of the two nanoscopy methods used in this thesis is better suited for neuronal imaging? This answer cannot be straightforward, as both STED and RESOLFT have unique benefits that cannot be provided by the other method. One main advantage of STED nanoscopy is the wide range of (well-known) fluorophores that are readily compatible with STED. The spectrum ranges from organic dyes over nitrogen-vacancy centers in nanodiamonds to fluorescent proteins and even reversibly switchable fluorescent proteins (RSFPs). Many of the commonly used fluorophores can be used, so that established labeling methods do not need to be reworked. Therefore, STED is a highly versatile approach that can be used to image most of the stable transgenic animal strains that have been engineered over the years, without requiring lengthy re-breeding processes. A second advantage of STED is the fast imaging speeds that are achievable. If the samples are bright enough, the pixel dwell times can be reduced to microseconds or even below. In this fashion, small frames $(3 \mu \mathrm{m} \times 3 \mu \mathrm{m})$ can be read out in tens of milliseconds and individual lines can be acquired in one millisecond or less. In our implementation we recorded entire stacks in under a minute; each stack typically consisted of 5 optical sections with frame sizes of $20 \mu \mathrm{m} \times 20 \mu \mathrm{m}$ and $30 \mathrm{~nm}$ pixels. The acquired images can be used and evaluated immediately, because the increase in resolution is a purely physical process and does not rely on any computational post-processing steps (as is the case for stochastic superresolution methods). This allows a high throughput of imaged structures, so that truly interesting locations can be identified quickly without wasting much time on acquiring images of suboptimal features.

RESOLFT nanoscopy surpasses STED by requiring much lower laser intensities, typically by five to six orders of magnitude. Not only does that alleviate the necessity for expensive, high-power lasers. More importantly for neuroscience, it enables neurons to be observed without exposing them to potentially damaging 
laser illumination. As demonstrated in this thesis, RESOLFT can be used to image highly sensitive structures such as synapses repeatedly without incurring any photo-induced effects. This is a huge advantage, as otherwise the validity of any results that were acquired using potentially fatal laser illumination can always be questioned. Even the widely used two-photon microscopes can easily induce fatal cell damage. The current RESOLFT implementation, however, relies on ultraviolet light, which is certainly not beneficial for cell viability or tissue penetration depth. But this limitation can be overcome by several approaches, such as using twophoton activation. For current RSFPs (such as Dronpa-M159T ${ }^{v 2.0}$ ), this two-photon light would be in the far-red spectral range, so in the so-called 'physiological window'. A further approach would be the development and use of red-shifted RSFPs.

The reliance on specific fluorescent labels, namely RSFPs, is a disadvantage, which reduces the flexibility and the amount of readily available samples, necessitating more time and effort for preparation. The main drawback of this RESOLFT implementation at the moment is the much slower imaging speed as compared to STED. Whereas the speed is fully sufficient to resolve even fast dynamic processes in the brain, it comes at the cost of a reduced field of view. This drawback can be overcome, however, either by developing faster-switching RSFPs or by parallelizing the setup, i.e. using multiple RESOLFT donuts at once to record multiple locations simultaneously. 


\section{Conclusions and outlook}

Optical far-field nanoscopy, i.e. optical microscopy with the power to resolve structures on the nanometer scale, has proven time and again that it can overcome the limitations imposed on spatial resolution by diffraction. In various laboratory settings, from fixed samples over live cells in culture ${ }^{49,144}$ to the surfaces of brain slices, ${ }^{70}$ optical nanoscopy has been used to reveal nanoscopic details that were previously not resolvable. But the full potential of these techniques for biological and neurological research can only be unlocked by using them to study the most interesting and promising biological samples, such as live multicellular organisms ${ }^{145}$ or intact neural networks. ${ }^{53}$ Despite the higher complexity of these samples, the nanoscopy techniques must retain their improved resolution and must not destroy or damage the living samples in any way. In this dissertation we demonstrated for the first time the capacity of targeted nanoscopy techniques to optically monitor morphological changes, sometimes for several hours, of postsynaptic structures belonging to a healthy neuron embedded deep inside the intact neural network of a living hippocampal brain slice.

Two separate setups were modified for transfecting, examining and also sustained nanoscopic imaging of living brain slices, composed around an inverse STED nanoscope and an inverse RESOLFT nanoscope. Both setups were equipped with a glycerol immersion objective lens, which enabled nanoscopic imaging even deep inside the dense neural tissue. The versatile STED nanoscope offered fast imaging speeds and was compatible with a wide range of fluorophores, from well-known fluorescent proteins like EGFP and EYFP to photoswitchable fluorescent proteins like Dronpa(-M159T) and Padron ${ }^{v 2.0} .{ }^{63,146}$ This setup was outfitted with heating and perfusion capabilities to sustain the brain slices over hours; enhanced transmission 
widefield contrast helped to visualize the hippocampal architecture and individual cells therein.

The speed of the RESOLFT setup needed to be enhanced, so as to enable time-lapse imaging of fast neuronal dynamics. By using the fast-switching Dronpa-M159T and optimizing the imaging parameters, we could improve the imaging speed by a factor of fifty as compared to other RSFPs. The RESOLFT setup offered two distinct advantages over the STED implementation. Because reversibly photoswitchable fluorescent proteins (RSFPs) were used as the nanoscopy-enabling on-off switch, the laser intensity required for switching was five to six orders of magnitude lower than for the STED setup. This reduced the light exposure of the imaged brain slices from potentially damaging levels to values at which no direct photodamage was visible and bleaching was negligible. The second advantage was the added capability to superresolve structures in three spatial dimensions, instead of only in the optical $(\mathrm{x}, \mathrm{y})$ plane. By combining two de-excitation beams with different phase patterns, we achieved a threefold isotropic resolution increase over confocal, diffraction limited values. ${ }^{147}$

Aberration compensation proved crucial for retaining high spatial resolutions even deep inside the brain slices. Even the simple methods implemented in this thesis proved to be sufficient to image in depths at which neurons had not been damaged in the cutting procedure and therefore retained intact synaptic connections. For the first time healthy dendritic spines were observed with $\leq 60 \mathrm{~nm}$ spatial resolution in depths up to $95 \mu \mathrm{m}$ in living brain slices and with $\leq 80 \mathrm{~nm}$ spatial resolution in depths up to $120 \mu \mathrm{m} .{ }^{148}$ This proved to be the maximum brain slice thickness that could be achieved with this preparation method. The high spatial resolution (in two or three dimensions) allowed structures and details to be discerned that were previously only resolvable by electron microscopy, such as the diameters of dendritic spine necks. Because the observed neurons were alive and healthy we could monitor the morphological responses of dendritic spine necks to postsynaptic activity directly for each spine as they occurred. The improved resolution allowed a much more accurate assessment of the volume and surface area of dendritic spines and the individual parts thereof under physiological conditions, without risking fixation artifacts such as overall shrinking, disruption of the actin cytoskeleton ${ }^{149}$ 
or other distortions that could potentially falsify the results. Conditions were established under which there was no appreciable bleaching of dye or photo-damage to the neurons. Thus it could be shown that even under steady-state conditions there were continuously fluctuations in spine position and morphology. This spine motility increased in frequency and magnitude at physiological temperatures. ${ }^{147}$

Spine neck diameters were monitored after neurons were chemically stimulated with a potentiation medium designed to elicit synaptic strengthening. On average the spine neck diameters of stimulated neurons increased by $\approx 30 \%$. These changes were completed in about 30 minutes after stimulation and remained stable thereafter for hours. Unstimulated spine necks also fluctuated, but the average neck width remained constant. Such direct observation of individual spine changes had not been possible previously, because electron micrographs are limited to comparing different populations of neurons and spine heads before and after stimulation.

In their current implementations, STED and RESOLFT are different nanoscopy techniques that are not in competition but rather complement each other. Both techniques have great potential for further improvement and development, especially the comparatively new RESOLFT methodology. If the imaging speed of RESOLFT nanoscopy can be increased through the development of new RSFPs and by parallelization and the dependence on UV light for switching can be eliminated, then RESOLFT would be the method of choice for noninvasively imaging sensitive neuronal dynamics because of its inherent low light levels. Very fast synaptic dynamics such as action potentials or ion channel dynamics will likely still be out of reach of RESOFLT nanoscopy, instead requiring the fast imaging speed s of STED to observe phenomena in the millisecond time range. Furthermore, due to the versatility and compatibility of STED nanoscopy with most of the commonly used fluorescent labels, ${ }^{150}$ STED will retain its usefulness for imaging (commonly labeled and most transgenic) neuronal samples in high resolution without the need to utilize RSFP labels.

For targeted nanoscopy approaches to truly establish their usefulness in neuroscience, further enhanced depth penetration will be required. Imaging depths between $200 \mu \mathrm{m}$ to $1000 \mu \mathrm{m}$ are common for imaging the various layers of the cerebral cortex in vivo with two-photon microscopy. To gain access to such depths, 
improvements that limit the influence of scattering, absorption and aberrations will be required. Aberrations can be tackled by adding further correction capabilities such as an objective lens that more closely matches the refractive index of brain tissue and, ideally, adaptive optics for actively correcting aberrations of even higher order. Scattering and absorption can be reduced by shifting to wavelengths in the red and near-infrared spectral range, thereby making use of the increased scattering and absorption mean pathways in that spectral range. For RESOLFT this entails the development of novel RSFPs with fast switching times, appropriate spectral properties and good photochemical properties. A shift in wavelength can also be achieved by implementing two-photon excitation and de-excitation beams. The short pulses and high peak power required for two-photon de-excitation processes, however, might well cause severe prohibitive photodamage to living samples. This would not be such a problem for RESOLFT nanoscopy, due to the overall lower illumination intensities required. Additionally, the optical sectioning capabilities inherent to two-photon microscopy would permit removing the confocal pinhole from the detection pathway, thereby increasing the effect of scattering by increasing the recorded signal. A combination of these individual approaches seems the most promising for enabling very deep imaging depths in living tissue.

STED and RESOLFT nanoscopy techniques open up whole new possibilities for research in the neurosciences. For the first time, these techniques enable the examination of hitherto unresolvable dynamic phenomena as they occur. This offers the unique possibility of studying the precise function of the dendritic spine neck, which is still much disputed. Nanoscopy has the potential to continue the series of groundbreaking discoveries that microscopy brought to the neurosciences, possibly enabling the next step towards unraveling the mysteries behind memory formation. 


\section{Bibliography}

1. E. Chudler. Milestones in neuroscience research. http://faculty.washington.edu/chudler/hist.html, 2004 (accessed September 14, 2012). [cited on p. 1]

2. E. Lazarides and K. Weber. Actin antibody: the specific visualization of actin filaments in nonmuscle cells. Proc Natl Acad Sci U S A, 71(6):2268-72, 1974. [cited on p. 3]

3. R. Y. Tsien. New calcium indicators and buffers with high selectivity against magnesium and protons: design, synthesis, and properties of prototype structures. Biochemistry, 19(11):2396404, 1980. [cited on p. 3]

4. L. M. Loew, L. Simpson, A. Hassner, and V. Alexanian. Unexpected blue shift caused by differential solvation of a chromophore oriented in a lipid bilayer. Journal of the American Chemical Society, 101(18):5439-5440, 1979. [cited on p. 3]

5. Per Brodal. The Central Nervous System. Oxford University Press, New York, second edition edition, 1998. [cited on pp. 3, 44,67]

6. E. Abbe. Beiträge zur theorie des mikroskops und der mikroskopischen wahrnehmung. Archiv für Mikroskopische Anatomie, 9:413-418, 1873. [cited on p. 4]

7. M. Knoll and E. Ruska. The electron microscope. Zeitschrift Fur Physik, 78(5-6):318-339, 1932. [cited on p. 5]

8. G. M. Shepherd. Neuron doctrine - revision of functional concepts. Yale Journal of Biology and Medicine, 45(6):584-599, 1972. [cited on p. 5]

9. E. G. Gray. Axo-somatic and axo-dendritic synapses of the cerebral cortex: an electron microscope study. J Anat, 93:420-33, 1959. [cited on p. 5]

10. E. G. Gray. Electron microscopy of synaptic contacts on dendrite spines of the cerebral cortex. Nature, 183(4675):1592-1593, 1959. [cited on pp. 5,77]

11. D. Kleinfeld, A. Bharioke, P. Blinder, D. D. Bock, K. L. Briggman, D. B. Chklovskii, W. Denk, M. Helmstaedter, J. P. Kaufhold, W. C. Lee, H. S. Meyer, K. D. Micheva, M. Oberlaender, S. Prohaska, R. C. Reid, S. J. Smith, S. Takemura, P. S. Tsai, and B. Sakmann. Large-scale automated histology in the pursuit of connectomes. The Journal of Neuroscience, 31(45):1612538, 2011. [cited on p. 6]

12. C. J. Sheppard and T. Wilson. Depth of field in the scanning microscope. Opt Lett, 3(3):115, 1978. [cited on p. 6]

13. I. J. Cox and C. J. Sheppard. Scanning optical microscope incorporating a digital framestore and microcomputer. Appl Opt, 22(10):1474, 1983. [cited on p. 6]

14. W. Denk, J. H. Strickler, and W. W. Webb. Two-photon laser scanning fluorescence microscopy. Science, 248(4951):73-6, 1990. [cited on pp. 7, 41,65]

15. M. Göppert-Mayer. Über elementarakte mit zwei quantensprüngen. Annalen der Physik, 401(3): 273-294, 1931. [cited on p. 7]

16. W. Kaiser and C. G. B. Garrett. 2-photon excitation in caf2 - eu2+. Phys Rev Lett, 7(6):229-231, 1961. [cited on p. 7] 
17. F. Helmchen and W. Denk. Deep tissue two-photon microscopy. Nature Methods, 2(12):932-40, 2005. [cited on pp. 7, 41, 63, 64,65]

18. K. Svoboda, W. Denk, D. Kleinfeld, and D. W. Tank. In vivo dendritic calcium dynamics in neocortical pyramidal neurons. Nature, 385(6612):161-5, 1997. [cited on p. 7]

19. S. Hell and E. H. K. Stelzer. Fundamental improvement of resolution with a 4 pi-confocal fluorescence microscope using 2-photon excitation. Optics Communications, 93(5-6):277-282, 1992. [cited on p. 8]

20. S. Hell and E. H. K. Stelzer. Properties of a 4pi confocal fluorescence microscope. Journal of the Optical Society of America a-Optics Image Science and Vision, 9(12):2159-2166, 1992. [cited on pp. ]

21. M. Schrader and S. W. Hell. 4pi-confocal images with axial superresolution. Journal of Microscopy-Oxford, 183:189-193, 1996. [cited on pp. ]

22. S. W. Hell, M. Schrader, and H. T. M. VanderVoort. Far-field fluorescence microscopy with three-dimensional resolution in the 100-nm range. Journal of Microscopy-Oxford, 187:1-7, 1997. [cited on p. 8]

23. M. G. L. Gustafsson, D. A. Agard, and J. W. Sedat. Sevenfold improvement of axial resolution in 3d widefield microscopy using 2 objective lenses. Three-Dimensional Microscopy: Image Acquisition and Processing Ii, 2412:147-156, 1995. [cited on p. 8]

24. M. G. L. Gustafsson, D. A. Agard, and J. W. Sedat. (im)-m-5: 3d widefield light microscopy with better than $100 \mathrm{~nm}$ axial resolution. Journal of Microscopy-Oxford, 195:10-16, 1999. [cited on p. 8]

25. G. Binnig and H. Rohrer. Scanning tunneling microscopy. Helvetica Physica Acta, 55(6):726-735, 1982. [cited on p. 8]

26. G. Binnig, C. F. Quate, and C. Gerber. Atomic force microscope. Phys Rev Lett, 56(9):930-933, 1986. [cited on p. 8]

27. D. P. Allison, N. P. Mortensen, C. J. Sullivan, and M. J. Doktycz. Atomic force microscopy of biological samples. Wiley Interdisciplinary Reviews-Nanomedicine and Nanobiotechnology, 2(6): 618-634, 2010. [cited on p. 8]

28. A. Lewis, H. Taha, A. Strinkovski, A. Manevitch, A. Khatchatouriants, R. Dekhter, and E. Ammann. Near-field optics: from subwavelength illumination to nanometric shadowing. Nature Biotechnology, 21(11):1377-1386, 2003. [cited on p. 8]

29. Q. Wu, G. D. Feke, R. D. Grober, and L. P. Ghislain. Realization of numerical aperture 2.0 using a gallium phosphide solid immersion lens. Applied Physics Letters, 75(26):4064-4066, 1999. [cited on p. 8]

30. S. W. Hell and J. Wichmann. Breaking the diffraction resolution limit by stimulated-emission - stimulated-emission-depletion fluorescence microscopy. Opt Lett, 19(11):780-782, 1994. [cited on p. 9]

31. S. W. Hell. Toward fluorescence nanoscopy. Nat Biotechnol, 21(11):1347-55, 2003. [cited on pp. 10,12]

32. S. W. Hell. Microscopy and its focal switch. Nature Methods, 6(1):24-32, 2009. [cited on pp. 10,66]

33. S. W. Hell, M. Dyba, and S. Jakobs. Concepts for nanoscale resolution in fluorescence microscopy. Curr Opin Neurobiol, 14(5):599-609, 2004. [cited on p. 12]

34. S. W. Hell. Far-field optical nanoscopy. Science, 316(5828):1153-8, 2007. [cited on p. 12]

35. S. W. Hell and M. Kroug. Ground-state-depletion fluorescence microscopy - a concept for breaking the diffraction resolution limit. Applied Physics B-Lasers and Optics, 60(5):495-497, 1995. [cited on p. 12]

36. S. W. Hell, S. Jakobs, and L. Kastrup. Imaging and writing at the nanoscale with focused visible light through saturable optical transitions. Applied Physics a-Materials Science \& Processing, 77 (7):859-860, 2003. [cited on p. 12] 
37. M. Hofmann, C. Eggeling, S. Jakobs, and S. W. Hell. Breaking the diffraction barrier in fluorescence microscopy at low light intensities by using reversibly photoswitchable proteins. Proceedings of the National Academy of Sciences of the United States of America, 102(49):1756517569, 2005. [cited on p. 12]

38. M. A. Schwentker, H. Bock, M. Hofmann, S. Jakobs, J. Bewersdorf, C. Eggeling, and S. W. Hell. Wide-field subdiffraction resolft microscopy using fluorescent protein photoswitching. Microsc Res Tech, 70(3):269-80, 2007. [cited on p. 12]

39. R. Heintzmann, T. M. Jovin, and C. Cremer. Saturated patterned excitation microscopy-a concept for optical resolution improvement. J Opt Soc Am A Opt Image Sci Vis, 19(8):1599-609, 2002. [cited on p. 12]

40. M. G. Gustafsson. Nonlinear structured-illumination microscopy: wide-field fluorescence imaging with theoretically unlimited resolution. Proc Natl Acad Sci U S A, 102(37):13081-6, 2005. [cited on p. 12]

41. E. Betzig, G. H. Patterson, R. Sougrat, O. W. Lindwasser, S. Olenych, J. S. Bonifacino, M. W. Davidson, J. Lippincott-Schwartz, and H. F. Hess. Imaging intracellular fluorescent proteins at nanometer resolution. Science, 313(5793):1642-5, 2006. [cited on p. 13]

42. S. T. Hess, T. P. Girirajan, and M. D. Mason. Ultra-high resolution imaging by fluorescence photoactivation localization microscopy. Biophysical Journal, 91(11):4258-72, 2006. [cited on p. 13]

43. M. J. Rust, M. Bates, and X. Zhuang. Sub-diffraction-limit imaging by stochastic optical reconstruction microscopy (storm). Nature Methods, 3(10):793-5, 2006. [cited on p. 13]

44. J. Folling, M. Bossi, H. Bock, R. Medda, C. A. Wurm, B. Hein, S. Jakobs, C. Eggeling, and S. W. Hell. Fluorescence nanoscopy by ground-state depletion and single-molecule return. Nature Methods, 5(11):943-5, 2008. [cited on p. 13]

45. M. Heilemann, S. van de Linde, M. Schuttpelz, R. Kasper, B. Seefeldt, A. Mukherjee, P. Tinnefeld, and M. Sauer. Subdiffraction-resolution fluorescence imaging with conventional fluorescent probes. Angew Chem Int Ed Engl, 47(33):6172-6, 2008. [cited on p. 13]

46. A. Egner, C. Geisler, C. von Middendorff, H. Bock, D. Wenzel, R. Medda, M. Andresen, A. C. Stiel, S. Jakobs, C. Eggeling, A. Schonle, and S. W. Hell. Fluorescence nanoscopy in whole cells by asynchronous localization of photoswitching emitters. Biophysical Journal, 93(9):3285-90, 2007. [cited on p. 13]

47. C. Geisler, A. Schonle, C. von Middendorff, H. Bock, C. Eggeling, A. Egner, and S. W. Hell. Resolution of lambda/10 in fluorescence microscopy using fast single molecule photo-switching. Applied Physics a-Materials Science \& Processing, 88(2):223-226, 2007. [cited on p. 13]

48. B. Huang, W. Wang, M. Bates, and X. Zhuang. Three-dimensional super-resolution imaging by stochastic optical reconstruction microscopy. Science, 319(5864):810-3, 2008. [cited on p. 14]

49. I. Izeddin, C. G. Specht, M. Lelek, X. Darzacq, A. Triller, C. Zimmer, and M. Dahan. Superresolution dynamic imaging of dendritic spines using a low-affinity photoconvertible actin probe. PLoS One, 6(1):e15611, 2011. [cited on pp. 14, 77, 104, 105,109]

50. T. V. P. Bliss and T. Lomo. Long-lasting potentiation of synaptic transmission in dentate area of anesthetized rabbit following stimulation of perforant path. Journal of Physiology-London, 232 (2):331-356, 1973. [cited on p. 17]

51. T. Hosokawa, D. A. Rusakov, T. V. Bliss, and A. Fine. Repeated confocal imaging of individual dendritic spines in the living hippocampal slice: evidence for changes in length and orientation associated with chemically induced Itp. The Journal of Neuroscience, 15(8):5560-5573, 1995. [cited on pp. 17, 40, 91,102]

52. B. H. Gahwiler, M. Capogna, D. Debanne, R. A. McKinney, and S. M. Thompson. Organotypic slice cultures: a technique has come of age. Trends in Neurosciences, 20(10):471-477, 1997. [cited on pp. 20, 21, 59,63] 
53. S. Berning, K. I. Willig, H. Steffens, P. Dibaj, and S. W. Hell. Nanoscopy in a living mouse brain. Science, 335(6068):551, 2012. [cited on pp. 20, 41, 104,109]

54. A. De Simoni, C. B. Griesinger, and F. A. Edwards. Development of rat ca1 neurones in acute versus organotypic slices: role of experience in synaptic morphology and activity. J Physiol, 550 (Pt 1):135-47, 2003. [cited on p. 21]

55. T. Grotjohann, I. Testa, M. Leutenegger, H. Bock, N. T. Urban, F. Lavoie-Cardinal, K. I. Willig, C. Eggeling, S. Jakobs, and S. W. Hell. Diffraction-unlimited all-optical imaging and writing with a photochromic gfp. Nature, 478(7368):204-208, 2011. [cited on pp. 29, 36, 37,98]

56. T. Brakemann, A. C. Stiel, G. Weber, M. Andresen, I. Testa, T. Grotjohann, M. Leutenegger, U. Plessmann, H. Urlaub, C. Eggeling, M. C. Wahl, S. W. Hell, and S. Jakobs. A reversibly photoswitchable gfp-like protein with fluorescence excitation decoupled from switching. Nature Biotechnology, 29(10):942-7, 2011. [cited on pp. 29,37]

57. B. Harke, C. K. Ullal, J. Keller, and S. W. Hell. Three-dimensional nanoscopy of colloidal crystals. Nano Letters, 8(5):1309-13, 2008. [cited on p. 31]

58. J. Riedl, A. H. Crevenna, K. Kessenbrock, J. H. Yu, D. Neukirchen, N. Bista, F. Bradke, D. Jenne, T. A. Holak, Z. Werb, M. Sixt, and R. Wedlich-Soldner. Lifeact: a versatile marker to visualize f-actin. Nature Methods, 5(7):605-607, 2008. [cited on pp. 33, 40,95]

59. M. Chalfie, Y. Tu, G. Euskirchen, W. W. Ward, and D. C. Prasher. Green fluorescent protein as a marker for gene expression. Science, 263(5148):802-5, 1994. [cited on p. 34]

60. O. Shimomura, F. H. Johnson, and Y. Saiga. Extraction, purification and properties of aequorin, a bioluminescent protein from the luminous hydromedusan, aequorea. J Cell Comp Physiol, 59: 223-39, 1962. [cited on p. 34]

61. R. Heim, D. C. Prasher, and R. Y. Tsien. Wavelength mutations and posttranslational autoxidation of green fluorescent protein. Proc Natl Acad Sci U S A, 91(26):12501-4, 1994. [cited on p. 34]

62. R. Heim, A. B. Cubitt, and R. Y. Tsien. Improved green fluorescence. Nature, 373(6516):663-4, 1995. [cited on p. 34]

63. A. C. Stiel, S. Trowitzsch, G. Weber, M. Andresen, C. Eggeling, S. W. Hell, S. Jakobs, and M. C. Wahl. 1.8 angstrom bright-state structure of the reversibly switchable fluorescent protein dronpa guides the generation of fast switching variants. Biochemical Journal, 402(1):35-42, 2007. [cited on pp. 34, 36,109]

64. R. Ando, H. Mizuno, and A. Miyawaki. Regulated fast nucleocytoplasmic shuttling observed by reversible protein highlighting. Science, 306(5700):1370-3, 2004. [cited on p. 36]

65. K. A. Lukyanov, A. F. Fradkov, N. G. Gurskaya, M. V. Matz, Y. A. Labas, A. P. Savitsky, M. L. Markelov, A. G. Zaraisky, X. Zhao, Y. Fang, W. Tan, and S. A. Lukyanov. Natural animal coloration can be determined by a nonfluorescent green fluorescent protein homolog. J Biol Chem, 275(34):25879-82, 2000. [cited on p. 36]

66. M. Andresen, A. C. Stiel, S. Trowitzsch, G. Weber, C. Eggeling, M. C. Wahl, S. W. Hell, and S. Jakobs. Structural basis for reversible photoswitching in dronpa. Proc Natl Acad Sci U S A, 104 (32):13005-9, 2007. [cited on p. 36]

67. J. K. Fazakerley. Pathogenesis of semliki forest virus encephalitis. J Neurovirol, 8 Suppl 2:66-74, 2002. [cited on p. 38]

68. T. Wakatsuki, B. Schwab, N. C. Thompson, and E. L. Elson. Effects of cytochalasin d and latrunculin b on mechanical properties of cells. J Cell Sci, 114(Pt 5):1025-36, 2001. [cited on pp. 40,72]

69. V. Westphal, S. O. Rizzoli, M. A. Lauterbach, D. Kamin, R. Jahn, and S. W. Hell. Video-rate far-field optical nanoscopy dissects synaptic vesicle movement. Science, 320(5873):246-9, 2008. [cited on p. 41]

70. U.V. Nägerl, K.I. Willig, S. W. Hein, S. W. Hell, and T. Bonhoeffer. Live-cell imaging of dendritic spines by sted microscopy. Proceedings of the National Academy of Sciences of the United States 
of America, 105(48):18982-18987, 2008. [cited on pp. 41,109]

71. N. A. Frost, H. Shroff, H. Kong, E. Betzig, and T. A. Blanpied. Single-molecule discrimination of discrete perisynaptic and distributed sites of actin filament assembly within dendritic spines. Neuron, 67(1):86-99, 2010. [cited on p. 41]

72. B. Huang, H. Babcock, and X. Zhuang. Breaking the diffraction barrier: super-resolution imaging of cells. Cell, 143(7):1047-58, 2010. [cited on pp. 41,42]

73. R. Yuste and W. Denk. Dendritic spines as basic functional units of neuronal integration. Nature, 375(6533):682-4, 1995. [cited on p. 41]

74. J. B. Ding, K. T. Takasaki, and B. L. Sabatini. Supraresolution imaging in brain slices using stimulated-emission depletion two-photon laser scanning microscopy. Neuron, 63(4):429-37, 2009. [cited on pp. 41,65]

75. G. Moneron and S. W. Hell. Two-photon excitation sted microscopy. Optics Express, 17(17): 14567-73, 2009. [cited on pp. 41,65]

76. W. Denk and K. Svoboda. Photon upmanship: why multiphoton imaging is more than a gimmick. Neuron, 18(3):351-7, 1997. [cited on p. 42]

77. P. Theer and W. Denk. On the fundamental imaging-depth limit in two-photon microscopy. J Opt Soc Am A Opt Image Sci Vis, 23(12):3139-49, 2006. [cited on pp. 43,65]

78. F. P. Bolin, L. E. Preuss, R. C. Taylor, and R. J. Ference. Refractive index of some mammalian tissues using a fiber optic cladding method. Appl Opt, 28(12):2297-303, 1989. [cited on pp. 43,44]

79. A. Brunsting and P. F. Mullaney. Differential light scattering from spherical mammalian cells. Biophysical Journal, 14(6):439-53, 1974. [cited on p. 44]

80. J. Bereiter-Hahn, C. H. Fox, and B. Thorell. Quantitative reflection contrast microscopy of living cells. J Cell Biol, 82(3):767-79, 1979. [cited on pp. ]

81. F. Lanni, A. S. Waggoner, and D. L. Taylor. Structural organization of interphase 3t3 fibroblasts studied by total internal reflection fluorescence microscopy. J Cell Biol, 100(4):1091-102, 1985. [cited on p. 44]

82. J. J. Dirckx, L. C. Kuypers, and W. F. Decraemer. Refractive index of tissue measured with confocal microscopy. J Biomed Opt, 10(4):44014, 2005. [cited on p. 44]

83. N. Lue, J. Bewersdorf, M. D. Lessard, K. Badizadegan, R. R. Dasari, M. S. Feld, and G. Popescu. Tissue refractometry using hilbert phase microscopy. Opt Lett, 32(24):3522-4, 2007. [cited on pp.]

84. T.K. Biwas and A.K. Gupta. Retrieval of true color of the internal organ of ct images and attempt to tissue characterization by refractive index : Initial experience. Ind J Radial Imag, 12(2):169178, 2002. [cited on p. 44]

85. J. J. Sun, S. J. Lee, L. Wu, M. Sarntinoranont, and H. K. Xie. Refractive index measurement of acute rat brain tissue slices using optical coherence tomography. Optics Express, 20(2):10841095, 2012. [cited on p. 44]

86. N. Martini, J. Bewersdorf, and S. W. Hell. A new high-aperture glycerol immersion objective lens and its application to 3d-fluorescence microscopy. J Microsc, 206(Pt 2):146-51, 2002. [cited on p. 46]

87. Leica Microsystems. Confocal application letter, resolution: Glycerol objective. Technical Report April 2004, No. 17, Leica Microsystems, 2004. [cited on p. 46]

88. S. Hell, G. Reiner, C. Cremer, and E. H. K. Stelzer. Aberrations in confocal fluorescence microscopy induced by mismatches in refractive-index. Journal of Microscopy-Oxford, 169:391405, 1993. [cited on pp. 48, 51,62]

89. M. J. Booth and T. Wilson. Refractive-index-mismatch induced aberrations in single-photon and two-photon microscopy and the use of aberration correction. J Biomed Opt, 6(3):266-72, 2001. [cited on pp. 48,65]

90. E. M. M. Manders. Chromatic shift in multicolour confocal microscopy. Journal of MicroscopyOxford, 185:321-328, 1997. [cited on p. 48] 
91. A. Egner, M. Schrader, and S. W. Hell. Refractive index mismatch induced intensity and phase variations in fluorescence confocal, multiphoton and 4pi-microscopy. Optics Communications, 153(4-6):211-217, 1998. [cited on p. 48]

92. M. J. Booth and T. Wilson. Strategies for the compensation of specimen-induced spherical aberration in confocal microscopy of skin. J Microsc, 200(Pt 1):68-74, 2000. [cited on p. 53]

93. T. Staudt, M. C. Lang, R. Medda, J. Engelhardt, and S. W. Hell. 2,2'-thiodiethanol: a new water soluble mounting medium for high resolution optical microscopy. Microsc Res Tech, 70(1):1-9, 2007. [cited on p. 55]

94. S. Berning. STED Nanoscopy of the Living Brain. PhD thesis, University of Göttingen, 2011. [cited on p. 55]

95. K. M. Harris and J. K. Stevens. Dendritic spines of ca 1 pyramidal cells in the rat hippocampus: serial electron microscopy with reference to their biophysical characteristics. The Journal of Neuroscience, 9(8):2982-97, 1989. [cited on pp. 56,61]

96. K. M. Harris and S. B. Kater. Dendritic spines: cellular specializations imparting both stability and flexibility to synaptic function. Annu Rev Neurosci, 17:341-71, 1994. [cited on pp. 56,61]

97. P. Hotulainen and C. C. Hoogenraad. Actin in dendritic spines: connecting dynamics to function. J Cell Biol, 189(4):619-29, 2010. [cited on pp. 62, 67,76]

98. A. N. Yaroslavsky, P. C. Schulze, I. V. Yaroslavsky, R. Schober, F. Ulrich, and H. J. Schwarzmaier. Optical properties of selected native and coagulated human brain tissues in vitro in the visible and near infrared spectral range. Physics in Medicine and Biology, 47(12):2059-2073, 2002. [cited on pp. 63,65]

99. L. Stoppini, P. A. Buchs, and D. Muller. A simple method for organotypic cultures of nervous tissue. J Neurosci Methods, 37(2):173-82, 1991. [cited on pp. 63,101]

100. P. Theer, M. T. Hasan, and W. Denk. Two-photon imaging to a depth of 1000 microm in living brains by use of a ti:al2o3 regenerative amplifier. Opt Lett, 28(12):1022-4, 2003. [cited on p. 64]

101. M. J. Booth. Adaptive optics in microscopy. Philos Transact A Math Phys Eng Sci, 365(1861): 2829-43, 2007. [cited on p. 65]

102. M. Schuster. Adaptive Optik in der STED-Mikroskopie - Verbesserung der Optik in dicken Proben. Diplom, Ruprecht-Karls-Universität Heidelberg, 2012. [cited on p. 65]

103. A. Holtmaat and K. Svoboda. Experience-dependent structural synaptic plasticity in the mammalian brain. Nat Rev Neurosci, 10(9):647-58, 2009. [cited on p. 67]

104. C. H. Bailey and E. R. Kandel. Structural changes accompanying memory storage. Annu Rev Physiol, 55:397-426, 1993. [cited on p. 67]

105. J. N. Bourne and K. M. Harris. Balancing structure and function at hippocampal dendritic spines. Annu Rev Neurosci, 31:47-67, 2008. [cited on p. 67]

106. J. Grutzendler, N. Kasthuri, and W. B. Gan. Long-term dendritic spine stability in the adult cortex. Nature, 420(6917):812-6, 2002. [cited on p. 67]

107. J. T. Trachtenberg, B. E. Chen, G. W. Knott, G. Feng, J. R. Sanes, E. Welker, and K. Svoboda. Long-term in vivo imaging of experience-dependent synaptic plasticity in adult cortex. Nature, 420(6917):788-94, 2002. [cited on p. 67]

108. T. A. Blanpied and M. D. Ehlers. Microanatomy of dendritic spines: emerging principles of synaptic pathology in psychiatric and neurological disease. Biol Psychiatry, 55(12):1121-7, 2004. [cited on p. 67]

109. P. Penzes, M. E. Cahill, K. A. Jones, J. E. VanLeeuwen, and K. M. Woolfrey. Dendritic spine pathology in neuropsychiatric disorders. Nat Neurosci, 14(3):285-93, 2011. [cited on p. 67]

110. M. Segal. Dendritic spines and long-term plasticity. Nat Rev Neurosci, 6(4):277-84, 2005. [cited on p. 67]

111. V. A. Alvarez and B. L. Sabatini. Anatomical and physiological plasticity of dendritic spines. Annu Rev Neurosci, 30:79-97, 2007. [cited on p. 67] 
112. M. Fischer, S. Kaech, D. Knutti, and A. Matus. Rapid actin-based plasticity in dendritic spines. Neuron, 20(5):847-54, 1998. [cited on pp. 67,104]

113. A. Dunaevsky, A. Tashiro, A. Majewska, C. Mason, and R. Yuste. Developmental regulation of spine motility in the mammalian central nervous system. Proc Natl Acad Sci U S A, 96(23): 13438-43, 1999. [cited on pp. 100, 104,105]

114. B. Lendvai, E. A. Stern, B. Chen, and K. Svoboda. Experience-dependent plasticity of dendritic spines in the developing rat barrel cortex in vivo. Nature, 404(6780):876-81, 2000. [cited on pp. 67,104]

115. H. Hering and M. Sheng. Dendritic spines: structure, dynamics and regulation. Nat Rev Neurosci, 2(12):880-8, 2001. [cited on pp. 67,75]

116. T. D. Pollard and G. G. Borisy. Cellular motility driven by assembly and disassembly of actin filaments. Cell, 112(4):453-65, 2003. [cited on p. 67]

117. K. Okamoto, T. Nagai, A. Miyawaki, and Y. Hayashi. Rapid and persistent modulation of actin dynamics regulates postsynaptic reorganization underlying bidirectional plasticity. Nat Neurosci, 7(10):1104-12, 2004. [cited on p. 85]

118. N. Honkura, M. Matsuzaki, J. Noguchi, G. C. Ellis-Davies, and H. Kasai. The subspine organization of actin fibers regulates the structure and plasticity of dendritic spines. Neuron, 57 (5):719-29, 2008. [cited on pp. 67, 85,106]

119. E. N. Star, D. J. Kwiatkowski, and V. N. Murthy. Rapid turnover of actin in dendritic spines and its regulation by activity. Nat Neurosci, 5(3):239-46, 2002. [cited on pp. 67,72]

120. R. Yuste and T. Bonhoeffer. Morphological changes in dendritic spines associated with longterm synaptic plasticity. Annu Rev Neurosci, 24:1071-89, 2001. [cited on p. 67]

121. H. Kasai, M. Matsuzaki, J. Noguchi, N. Yasumatsu, and H. Nakahara. Structure-stabilityfunction relationships of dendritic spines. Trends in Neurosciences, 26(7):360-8, 2003. [cited on pp.]

122. A. Matus, H. Brinkhaus, and U. Wagner. Actin dynamics in dendritic spines: a form of regulated plasticity at excitatory synapses. Hippocampus, 10(5):555-60, 2000. [cited on pp. ]

123. L. A. Cingolani and Y. Goda. Actin in action: the interplay between the actin cytoskeleton and synaptic efficacy. Nat Rev Neurosci, 9(5):344-56, 2008. [cited on p. 67]

124. G. Feng, R. H. Mellor, M. Bernstein, C. Keller-Peck, Q. T. Nguyen, M. Wallace, J. M. Nerbonne, J. W. Lichtman, and J. R. Sanes. Imaging neuronal subsets in transgenic mice expressing multiple spectral variants of gfp. Neuron, 28(1):41-51, 2000. [cited on p. 69]

125. J. Riedl, K. C. Flynn, A. Raducanu, F. Gartner, G. Beck, M. Bosl, F. Bradke, S. Massberg, A. Aszodi, M. Sixt, and R. Wedlich-Soldner. Lifeact mice for studying f-actin dynamics. Nature Methods, 7 (3):168-9, 2010. [cited on p. 69]

126. I. H. Chen, S. W. Chu, C. K. Sun, P. C. Cheng, and B. L. Lin. Wavelength dependent damage in biological multi-photon confocal microscopy: A micro-spectroscopic comparison between femtosecond ti : sapphire and cr : forsterite laser sources. Optical and Quantum Electronics, 34 (12):1251-1266, 2002. [cited on p. 72]

127. I. Spector, N. R. Shochet, Y. Kashman, and A. Groweiss. Latrunculins: novel marine toxins that disrupt microfilament organization in cultured cells. Science, 219(4584):493-5, 1983. [cited on p. 72]

128. M. Coue, S. L. Brenner, I. Spector, and E. D. Korn. Inhibition of actin polymerization by latrunculin a. FEBS Lett, 213(2):316-8, 1987. [cited on p. 72]

129. I. Spector, N. R. Shochet, D. Blasberger, and Y. Kashman. Latrunculins-novel marine macrolides that disrupt microfilament organization and affect cell growth: I. comparison with cytochalasin d. Cell Motil Cytoskeleton, 13(3):127-44, 1989. [cited on p. 72]

130. D. W. Allison, V. I. Gelfand, I. Spector, and A. M. Craig. Role of actin in anchoring postsynaptic receptors in cultured hippocampal neurons: differential attachment of nmda versus ampa receptors. The Journal of Neuroscience, 18(7):2423-36, 1998. [cited on pp. 72,103] 
131. K. E. Sorra and K. M. Harris. Overview on the structure, composition, function, development, and plasticity of hippocampal dendritic spines. Hippocampus, 10(5):501-11, 2000. [cited on p. 75]

132. M. Roelandse, A. Welman, U. Wagner, J. Hagmann, and A. Matus. Focal motility determines the geometry of dendritic spines. Neuroscience, 121(1):39-49, 2003. [cited on p. 75]

133. M. Renner, C. G. Specht, and A. Triller. Molecular dynamics of postsynaptic receptors and scaffold proteins. Curr Opin Neurobiol, 18(5):532-40, 2008. [cited on p. 76]

134. F. Korobova and T. Svitkina. Molecular architecture of synaptic actin cytoskeleton in hippocampal neurons reveals a mechanism of dendritic spine morphogenesis. Mol Biol Cell, 21(1):165-76, 2010. [cited on pp. 76, 82,106]

135. Y. Inoue, H. Udo, K. Inokuchi, and H. Sugiyama. Homer1a regulates the activity-induced remodeling of synaptic structures in cultured hippocampal neurons. Neuroscience, 150(4):84152, 2007. [cited on p. 96]

136. B. Xiao, J. C. Tu, R. S. Petralia, J. P. Yuan, A. Doan, C. D. Breder, A. Ruggiero, A. A. Lanahan, R. J. Wenthold, and P. F. Worley. Homer regulates the association of group 1 metabotropic glutamate receptors with multivalent complexes of homer-related, synaptic proteins. Neuron, 21(4):707-16, 1998. [cited on p. 96]

137. E. H. Rego, L. Shao, J. J. Macklin, L. Winoto, G. A. Johansson, N. Kamps-Hughes, M. W. Davidson, and M. G. Gustafsson. Nonlinear structured-illumination microscopy with a photoswitchable protein reveals cellular structures at 50-nm resolution. Proc Natl Acad Sci U S A, 109(3):E135-43, 2012. [cited on p. 98]

138. S. Halpain. Actin and the agile spine: how and why do dendritic spines dance? Trends in Neurosciences, 23(4):141-6, 2000. [cited on p. 103]

139. E. Korkotian and M. Segal. Regulation of dendritic spine motility in cultured hippocampal neurons. The Journal of Neuroscience, 21(16):6115-24, 2001. [cited on pp. 104,105]

140. E. Rittweger, K. Y. Han, S. E. Irvine, C. Eggeling, and S. W. Hell. Sted microscopy reveals crystal colour centres with nanometric resolution. Nature Photonics, 3(3):144-147, 2009. [cited on p. 106]

141. E. Rittweger, D. Wildanger, and S. W. Hell. Far-field fluorescence nanoscopy of diamond color centers by ground state depletion. Epl, 86(1), 2009. [cited on p. 106]

142. X. Hu, C. Viesselmann, S. Nam, E. Merriam, and E. W. Dent. Activity-dependent dynamic microtubule invasion of dendritic spines. The Journal of Neuroscience, 28(49):13094-105, 2008. [cited on p. 106]

143. X. Hu, L. Ballo, L. Pietila, C. Viesselmann, J. Ballweg, D. Lumbard, M. Stevenson, E. Merriam, and E. W. Dent. Bdnf-induced increase of psd-95 in dendritic spines requires dynamic microtubule invasions. The Journal of Neuroscience, 31(43):15597-603, 2011. [cited on p. 106]

144. B. Hein, K. I. Willig, and S. W. Hell. Stimulated emission depletion (sted) nanoscopy of a fluorescent protein-labeled organelle inside a living cell. Proc Natl Acad Sci U S A, 105(38): 14271-6, 2008. [cited on p. 109]

145. B. R. Rankin, G. Moneron, C. A. Wurm, J. C. Nelson, A. Walter, D. Schwarzer, J. Schroeder, D. A. Colon-Ramos, and S. W. Hell. Nanoscopy in a living multicellular organism expressing gfp. Biophysical Journal, 100(12):L63-5, 2011. [cited on p. 109]

146. K. I. Willig, A. C. Stiel, T. Brakemann, S. Jakobs, and S. W. Hell. Dual-label sted nanoscopy of living cells using photochromism. Nano Letters, 11(9):3970 - 3973, 2011. [cited on p. 109]

147. I. Testa, N. T. Urban, S. Jakobs, C. Eggeling, K. I. Willig, and Stefan W. Hell. Nanoscopy of living brain slices with low light levels. Nuron, 75(6):992-1000, 2012. [cited on pp. 110,111]

148. N. T. Urban, K. I. Willig, S. W. Hell, and U. V. Nagerl. Sted nanoscopy of actin dynamics in synapses deep inside living brain slices. Biophysical Journal, 101(5):1277-84, 2011. [cited on p. 110] 
149. E. Urban, S. Jacob, M. Nemethova, G. P. Resch, and J. V. Small. Electron tomography reveals unbranched networks of actin filaments in lamellipodia. Nat Cell Biol, 12(5):429-35, 2010. [cited on p. 110]

150. K. I. Willig, R. R. Kellner, R. Medda, B. Hein, S. Jakobs, and S. W. Hell. Nanoscale resolution in gfp-based microscopy. Nature Methods, 3(9):721-3, 2006. [cited on p. 111] 



\section{A. Appendix}

\section{Spine neck measurements}

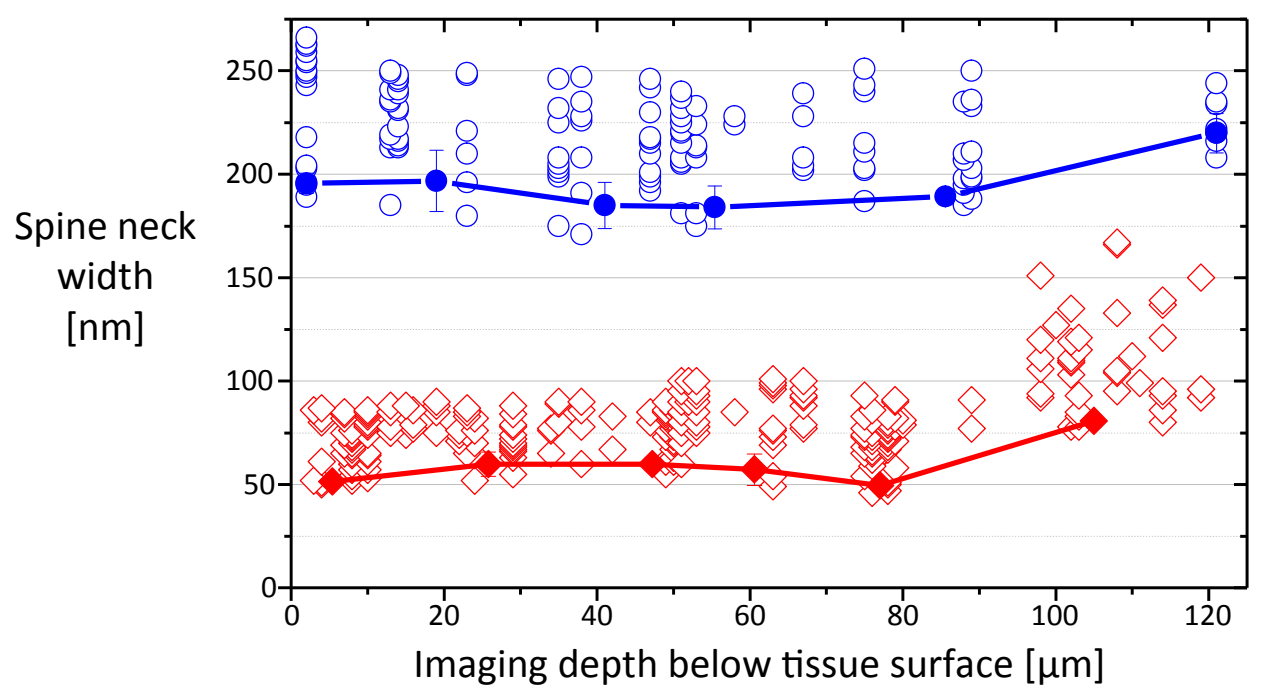

Figure A.1. | Spine neck diameters measured at various depths inside brain slices. Displayed are up to 50 of the thinnest spine necks (FWHMs) per data bin for both confocal (open circles) and STED (open diamonds). An average of the five thinnest values per bin is displayed as a guide to the eye for confocal (closed blue circles) and STED (closed red diamonds). Resolutions of at least $190 \mathrm{~nm}$ for confocal and $60 \mathrm{~nm}$ for STED could be sustained up until depths of $95 \mu \mathrm{m}$ after which the aberration correction capabilities reached their limits. The resolution declined gradually from here on; the maximum recorded depth was $120 \mu \mathrm{m}$. 


\section{Depth penetration in RESOLFT}

Figure A.2 | Simple aberration correction techniques enables deeper imaging with RESOLFT. By adjusting the correction collar of our objective lens (between a correction value of 5.5 and 7) to counteract the aberrations occurring at various imaging depths, we were able to acquire RESOLFT images at different depths within the brain sample; here, at (A) $10 \mu \mathrm{m}$, (B) $30 \mu \mathrm{m}$ and (C) $50 \mu \mathrm{m}$ inside living brain slices, respectively. These images were recorded with sub-diffraction resolution in two-dimensions only. Over this entire range we did not observe any image degradation in terms of signal or resolution impairment. The pixel size in (A) was $30 \mathrm{~nm}$, and in (B) and (C) $25 \mathrm{~nm}$.

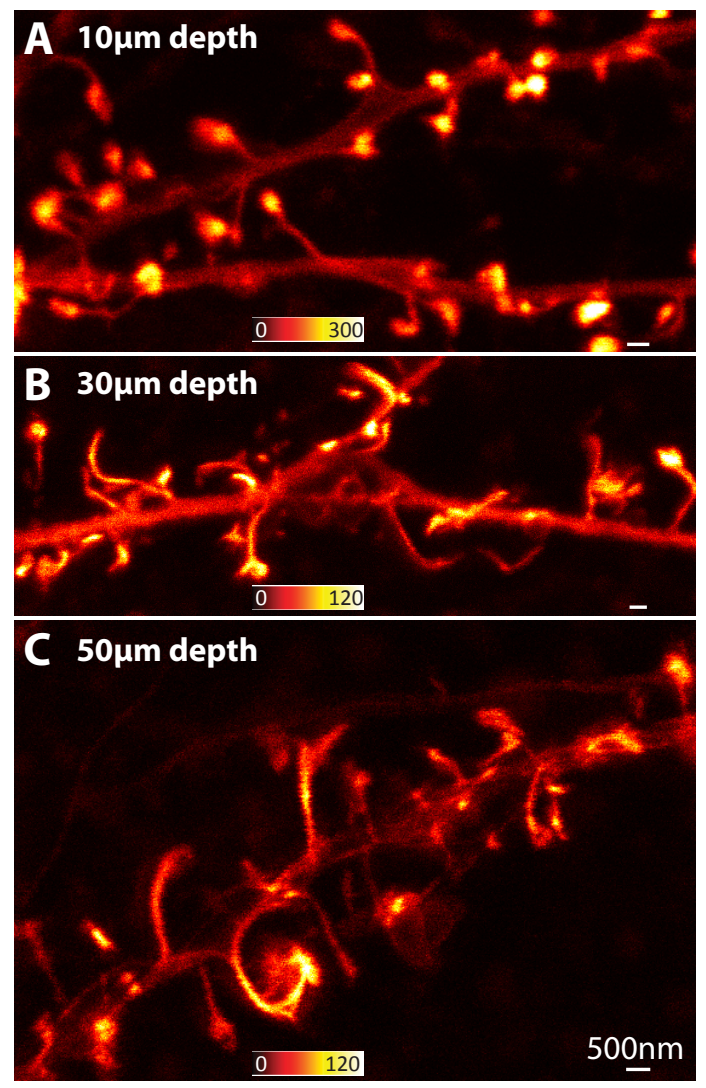




\section{Additional spine parameters measured after LTP}
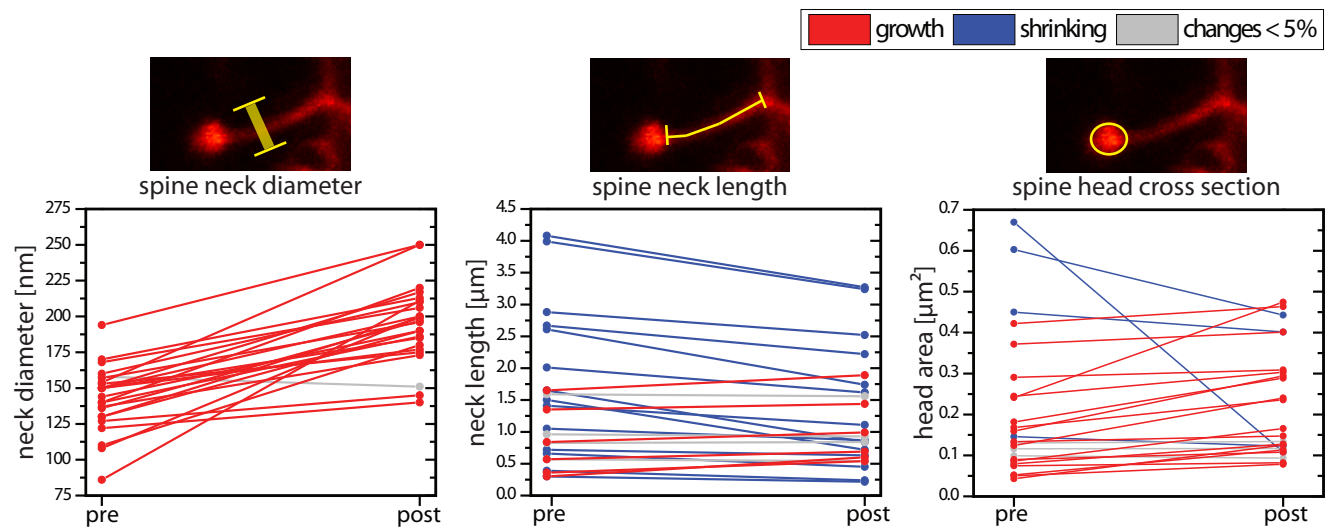

Figure A.3. | Morphological changes of dendritic spines were quantified before and after LTP stimulation. We analyzed the morphology of 24 dendritic spines from two different brain slices before and after LTP stimulation. All increases are denoted in red, all decreases in blue and all cases with little to no visible changes in dimension are marked in grey. (A) Spine neck widths were measured by determining the FWHMs of 3-pixel-wide line intensity profile that were taken from the same position before and after stimulation. (B) Spine neck lengths were measured from the spine base anchored to the dendritic shaft up to the beginning of the spine head. (C) The area, or cross section, of the spine heads was determined by drawing a bounding box around all pixels over a certain threshold in the spine head and determining the resulting surface area. All these analyses were performed with Image J. 


\section{Repeated RESOLFT imaging of a non-replenishing fluorescent label}

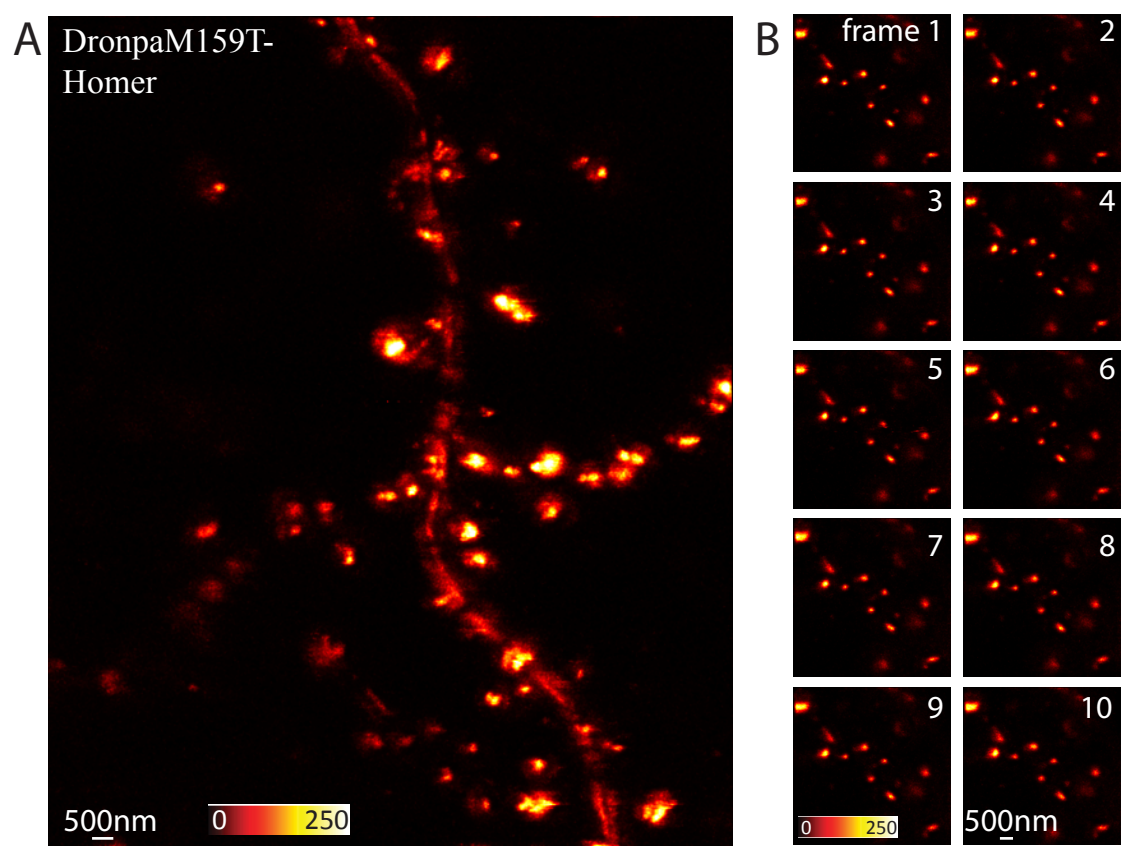

Figure A.4. | Repeated imaging of a non-replenishing postsynaptic protein label. Dendritic spines were labeled using a Dronpa-M159T-HOMER1c fusion protein, which labels the postsynaptic density in a non-replenishing manner. (A) In an overview picture $(19.5 \mu \mathrm{m} \times 19.5 \mu \mathrm{m}$, pixel size $30 \mathrm{~nm}, 5$ optical sections) the Homer1c protein can be seen to cluster in spots of various sizes along the dendritic shaft. (B) Repeated imaging of a smaller area $((6.6 \mu \mathrm{m} \times 6.6 \mu \mathrm{m}$, pixel size $30 \mathrm{~nm}$, no projection) displays only marginal bleaching after ten frames, despite the label not being able to replenish during the imaging. The lookup table is identical for all for each single image. We repeated this imaging scheme at different locations for up to 100 frames, observing a $50 \%$ bleaching after about 40 frames. These pictures were deconvolved using a Richardson-Lucy algorithm. 


\section{Acknowledgements}

These last three and a half years have been an incredible experience for me. It is truly awe-inspiring to work every day with such a talented, intelligent and tenacious group of individuals. At times it makes you feel like a bumbling, ignorant fool in comparison, but in a good way. I have learned much, done much and was allowed to take part and participate in cutting edge research.

First and foremost, I owe my gratitude to Prof. Stefan W. Hell who generously gave me this opportunity to conduct research in a field of science in which I had no prior knowledge, training or expertise. His firm support, helpful and concise advice and visions for the future were well received. I greatly admire his unconditional stance on maintaining the highest standards of good scientific practice.

I would like to thank Prof. Erwin Neher and Prof. Jörg Enderlein for taking great interest in my project and for advising me throughout the course of my thesis work. Your critiques, comments and suggestions provided good insights and helped me put my research into the broader context.

To Katrin Willig I owe considerable gratitude for guiding me on the rocky path that is independent research. She invested considerable time and effort into my research, helped whenever there was a problem and always had time to discuss the projects. It might not have always been easy, but after all the best advice you can receive is typically not the easiest.

I have benefited greatly from Valentin Nägerl who assisted with my first steps into the field of neuroscience. His advice, ideas and knowledge on all things brainy were helpful when the neurons were, once again, not behaving as they should. His boundless enthusiasm and optimism was also very catching. 
Without Tanja Gilat my life would have been considerably more tedious and frustrating. She assisted and accompanied me throughout innumerous brain slice preparations, lending a helping hand when required, providing everything I needed when I needed it and taking care of some of the nastier, more taxing jobs that had to be done. That and help preserve my sanity and my sense of humor when I had to conduct 12-hour bouts of non-stop brain surgery.

Frank Voss taught me most of everything I know about cutting, preparing and evaluating hippocampal brain slices. I profited greatly from his experience every time he offered me advice on a problem with the brain slices I could not quite pinpoint on my own.

It has been a great pleasure working with Ilaria Testa who is a true wizard when it comes to constructing, aligning and rebuilding optical setups. Without her RESOLFT nanoscope there would have been considerably fewer pictures in this thesis.

I profited from my discussion and deliberations with Sebastian Berning on setups, ideas, techniques, mice, presentations and good whisky. He also helped me learn the basics of planning, adjusting and optimizing optical microscopes. Thanks!

Considerable thanks go to Jaydev Jethwa who always has a smile on his face and a kind word on his lips. He is the guy to go to when you need something. And if he can't help you, then he just might know a guy, who knows a guy, who just might be able to help...

Harald Meyer assisted greatly with the electronics required for my electrophysiology equipment and projects, which unfortunately had not been concluded in time for me to present them in this thesis. Marco Roose is a wizard with all things IT-related and has to date not failed in providing me with a quick and easy solution, replacement, improvement or general-something to make my life a little easier. Thanks to Christian Dietl who always provided me with mice when I needed them, and thanks to Ulrike Teichmann for ensuring the well-being of our lab animals.

Thanks go to Sarah Leondarakis, Julia Guhlich, Silvia Schirmer, Alena Stephan and Josephine Stadler who somehow manage to keep this group organized while taking care of any and all forms, timetables, contracts, travel arrangements, letters, packages, schedules and much more. And somehow they never lost their calm or their smiles.

Several charming individuals deserve special acknowledgement, simply for being really good friends. Thanks, Débora Machado Andrade for always providing a smile and an open ear and for being super excited and giddy when I share good news with you. Franziska 
Brunner is always good for a laugh, a rant, beer, utter nonsense, jogging, general advice and sometimes even scientific discussion. Thanks to Lena Große for teaching me the relevance of Star Wars for scientific publications, boosting difficult days with chocolate and sweets and for being someone to geek-out with. A shout-out goes to my meat-loving pal Dirk Kamin for a great sense of humor, good advice on brainy stuff and general awesomeness. Express thanks go to Veronika Müller for always helping out when I needed expert advice or support and for being the best Christmas-pageant-director there is, to Ulrike Böhm for good discussions and lots of support, to Jakob Bierwagen especially for dragging me the Göttinger Stadtkantorei, to Christian Wurm for casual discussions, insights and advice, and to Mark Bates and Daniel Jans for being devoted Hell's Runners. For brevity, special thanks to Sebastian, Martin, Johann, Rebecca, Susann, Flavie, Heiko, Ellen, Chaitanya, Brian, Christian and many others, who make this group such a great place to work in!

A big shout-out to the best office-mates (past and present) in tower two, namely Doug, Haisen, Andriy, Fabian, Elisa, Dirk, Nina, Giuseppe and Kyu-Young.

Special thanks go to my proofreaders Dirk Kamin, Katrin Willig, Alexander and Cordula Urban and my ever-vigilant parents for finding and eliminating most of the nonsense that constantly tried to sneak into my dissertation.

I owe a special debt to my friends in Karlsruhe and to Hüttens in particular. You help keep me grounded whenever I descend too far into the quagmire of learning and scientific research and never fail to provide an excuse to celebrate. After all, there can be no better reason than because we can. Some people deserve special mention because they are awesome. I am thinking of you, Nimms, Seuv, Spock, Feuer, Gips, Fern, Heim, Sisko and Swing (to mention a few).

On a separate note, I want Dany and Obi to know that I regard them as great scientific role models. You have helped me a great deal, probably more than either of you suspect. And, as promised, your dissertations (which have been sitting on my desk for some months now) have been a motivation to give it my all! Thanks a lot. Almost equally impressive is Dominic, who has shown that you can achieve anything, no matter the odds. Just do your best, never get discouraged and learn when not to listen to complete imbeciles.

My entire family is and has been an inexhaustible source of comfort and support without whom I would not be who I am. Even though I cannot thank you enough, I'll give it a try. 
Endless gratitude belongs to Franzi, who is my counterweight and my balance. Who unfailingly believed in me and inspired me to do my very best every day. You know that someone truly loves you, when they value your happiness over their own.

I am deeply grateful to my newly gained parents who have shown me nothing but kindness, love and support as if I had always been part of the family. I am very pleased to have gained such a great extended family, parents, brother, sister-in-law, niece, dog and all.

My brothers Alexander and Dominic have stuck by me through many crazy endeavors. Always supportive, they have helped me up, whenever I was down. Of course, when I was standing they never hesitated to pound me back down to the ground. Or at least take me down a notch or two. Well... they try, at least.

Where would I be without Brooby and the gang, who have been loyal, supportive and a general nuisance for years on end. Yes, I know that you could have written this thesis much faster, better and wittier than I ever could have managed. But hey, I am only human!

To my beloved grandmother in Wales I am deeply grateful She always sends her best wishes, hopes and prayers when she knows that I need them, and also when not. Also, she is tough as nails, as fierce as a Viking battle-axe and the most stubbornly steadfast person I know.

To my parents I owe devout thanks, who have never, ever failed me when I needed them. Who gave me an ideal to strive for, by just being who they were and leading by example. Who showed me how to be compassionate to everyone and anyone, as it is our duty in life to help others who need us. I owe my mother for teaching me the love of words. And especially for demonstrating that what must be endured, can be endured. So there is no point whining or sulking about it, just keep smiling and do your best. My father has been inspirational, demonstrating every day that as long as you do what you love doing, no minute of your life goes wasted. When you work, work hard. When you are off-duty, stop working and concentrate on relaxing, partying and being with friends and family.

Two better people I may never know.

I am truly sorry for anyone I have omitted who is deserving of my gratitude. I am also sorry for rambling on so much, but whoever really knows me also knows that this is what I do. Drama, baby, drama! 